UC-721

Issued: May 1995

Tank SY-102 Remediation Project

Summary Report: ASPEN Modeling

W. A. Punjak

S. B. Schreiber

S. L. Yarbro

DSTRBUTION OF THS OOCUNEA IS UNLIMTEO Git 


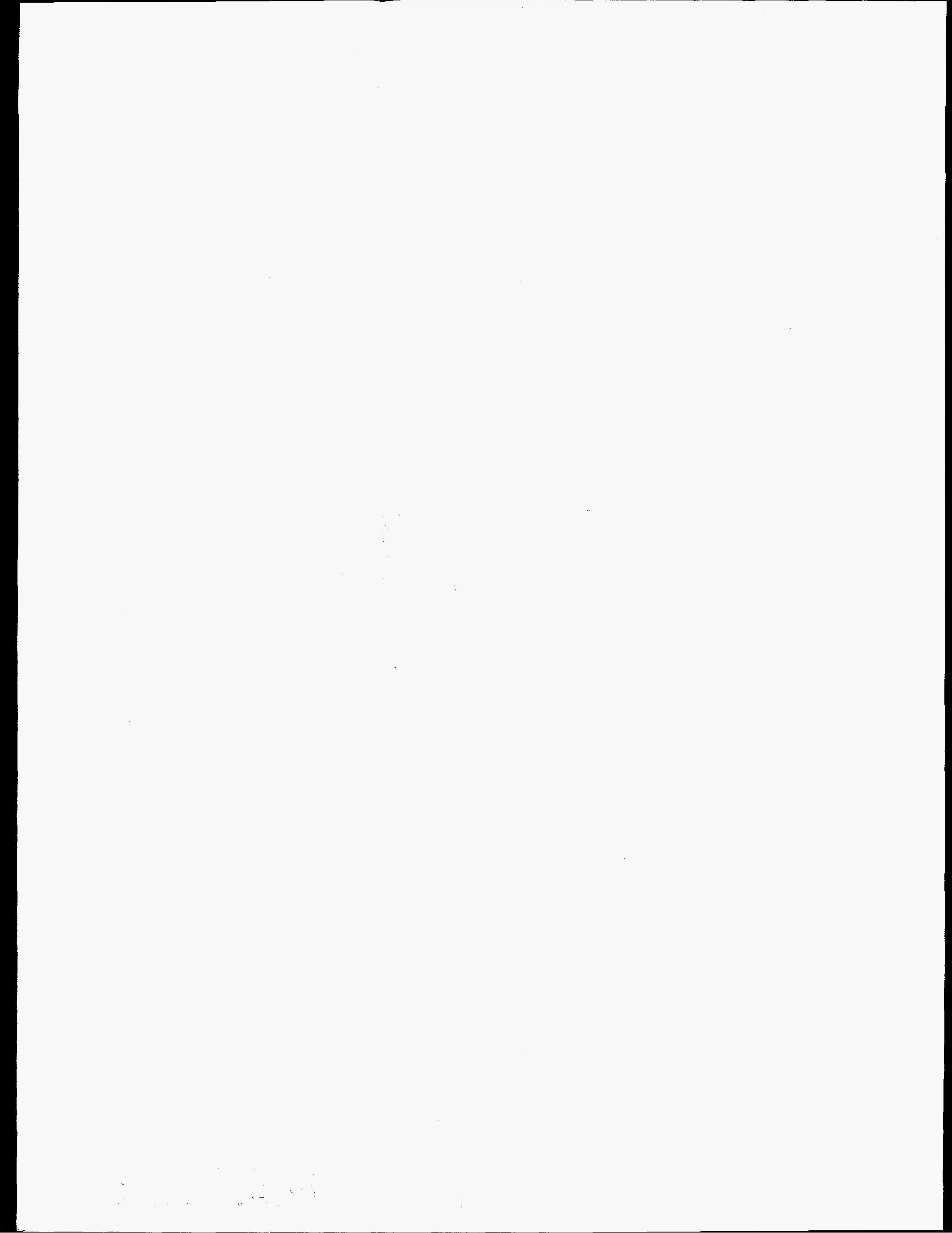




\section{DISCLAIMER}

This report was prepared as an account of work sponsored by an agency of the United States Government. Neither the United States Government nor any agency thereof, nor any of their employees, make any warranty, express or implied, or assumes any legal liability or responsibility for the accuracy, completeness, or usefulness of any information, apparatus, product, or process disclosed, or represents that its use would not infringe privately owned rights. Reference herein to any specific commercial product, process, or service by trade name, trademark, manufacturer, or otherwise does not necessarily constitute or imply its endorsement, recommendation, or favoring by the United States Government or any agency thereof. The views and opinions of authors expressed herein do not necessarily state or reflect those of the United States Government or any agency thereof. 


\section{DISCLAIMER}

Portions of this document may be illegible in electronic image products. Images are produced from the best available original document. 


\title{
Tank SY-102 Remediation Project Summary Report: ASPEN Modeling
}

by

W. A. Punjak, S. B. Schreiber, and S. L. Yarbro

\begin{abstract}
The U.S. Department of Energy established the Tank Waste Remediation System (TWRS) to safely manage and dispose of radioactive waste stored in underground tanks on the Hanford Site. As a part of this program, personnel at Los Alamos National Laboratory (LANL) have developed and demonstrated a flow sheet to remediate tank SY-102, which is located in the 200 West Area and contains high-level radioactive waste. In the conceptual design report issued earlier, an ASPEN Plus ${ }^{\mathrm{TM}}$ computer model of the flow sheet was presented. This report documents improvements in the flow sheet model after additional thermodynamic data for the actinide species were incorporated.
\end{abstract}

\section{Introduction}

Several reports covering the development of a flow sheet at Los Alamos National Laboratory (LANL) to process the contents of tank 102-SY at Westinghouse Hanford Company (WHC) have been written during the past couple years. They include an interim progress report (Yarbro et al. 1994), a conceptual design report (Yarbro et al. 1994), and a flow sheet scale-up report (Punjak et al. 1994). The first two reports describe the history and rationale for processing the contents of 102-SY, as well as how a flow sheet was selected and demonstrated on the bench scale. The conceptual design report also includes a working material flow model (using ASPEN Plus ${ }^{\mathrm{TM}}$ modeling software), a major equipment list, and cost estimates. The scale-up report describes experiments performed at LANL to show that the flow sheet can be scaled up predictably. This report documents improvements to the ASPEN Plus ${ }^{T M}$ material flow model. For information on other aspects of the 102-SY flow sheet development the reader is referred to the appropriate report(s) mentioned above. 


\section{Background}

An initial "first pass" ASPEN Plus ${ }^{T M}$ flow sheet model was included in the conceptual design report (Yarbro et al. 1994). That initial effort was primarily limited to defining stream flows through an overall material balance. All streams were modeled as aqueous electrolyte liquids and the only chemical reaction included was that $\mathrm{H}_{3} \mathrm{O}^{+}$ and $\mathrm{OH}^{-}$to make $\mathrm{H}_{2} \mathrm{O}$. The reasons for using this simple approach are outlined in the following paragraphs.

The version of ASPEN Plus ${ }^{\mathrm{TM}}$ used in the conceptual design report was release 8.5-6, the most recent version available at that time. Although release 8.5-6 can handle electrolyte systems, it requires user input for the electrolyte chemistry including the formation of precipitating salts. Choosing the appropriate reactions to include in the model is a formidable task when a large number of species are present, as is the situation with tank 102-SY. Therefore, only the acid-base neutralization reaction was included.

Another problem is the lack of physical parameter data for the actinides. The ASPEN Plus ${ }^{\text {TM }}$ data banks available with release 8.5-6 contain very little of the actinide parameter data required for the program to run. Although the literature contains much of the required clata, collecting it for input to an ASPEN Plus ${ }^{T M}$ user data bank can be quite time-consuming. In addition, much of the data required is not available and must be estimated.

Finally, ASPEN Plus ${ }^{\mathrm{TM}}$ does not contain an ion exchange model that is adequate for the proposed flow sheet. This is not surprising, since the nitrate anion exchange of actinides is a very complex and not completely understood process. This latter problem was circumvented by collecting experimental data on the bench and pilot scale. The ion exchange unit operation was then modeled in ASPEN Plus TM as a stream separator with defined splits based on the experimental data.

\section{Current Modeling Effort}

At the time the conceptual design report was published, researchers at New Mexico State University were compiling extensive thermodynamic data to be used to assemble a more realistic simulation. At about the same time, ASPEN Technology made available release 9.1-3, a version with many improvements. These improvements include an electrolytes chemistry generator that is able to compile most of the possible 
chemical reactions given a set of electrolyte species, together with various salts that may precipitate during reaction. The new release also has an enhanced physical property data bank that contains thermodynamic information on many of the actinide compounds. With the availability of the data from New Mexico State University (Mostafa et al. 1995), a more realistic model of the flow sheet was assembled.

The latest iteration of the flow sheet model still required some assumptions to be made. This was necessary to "fill in the gaps" where information was missing or, in one case, reduce the amount of information so that the model could run in a reasonable period of time. An example of the latter case was the initial use of the electrolytes chemistry generator, which came up with over 40 reactions. Only the major reactions were kept (listed in the report summary); the others were deleted so that the program would be manageable. It was also necessary to add some reactions that the chemistry generator did not make, including the formation of actinide hydroxides and oxides.

The process flow diagram (PFD) for the latest iteration is shown in Figure 1, and a stream summary for the simulation is shown in Figure 2. A description of each unit operation is given in Table 1. The ASPEN Plus ${ }^{\mathrm{TM}}$ report file is also included. Referring to the PFD and the stream summary, the composition of stream 1 is based on the current knowledge of the tank contents, as listed in the conceptual design report. A split of 5:1 liquid-to-solid ratio is assumed in the tank. The composition of streams 2 and 3 are based on the sludge washing studies of Lumetta and Swanson (1992). The washed sludge is dissolved in concentrated $\mathrm{HNO}_{3}$ to yield a slightly acidic $\left(0.05 \mathrm{M} \mathrm{H} \mathrm{H}^{+}\right)$product. The actinides are then removed by anion exchange, followed by strontium removal by ion exchange in an additional unit. The resulting stream (16) is combined with the supernate stream (27) after the cesium and technetium have been removed by cation and anion exchange, respectively. The actinide products from anion exchange are precipitated with $\mathrm{NaOH}$, then calcined to an oxide. The caustic liquid from the precipitation is combined with streams 16 and 27.

Streams 15, 24, and 26 represent the removal of strontium, cesium, and technetium, respectively, by the removal of the loaded resin. As with the first iteration, the frequency and amount of backflush acid for the filter F-S-1 is not known at this stage of design. Therefore the flow rates for streams 6 and 9 are not listed in the stream summary. Based on the input parameters specified, the combination of streams 16, 19, and 27 result in a solution caustic enough for direct cementation. However, due to uncertainties in the various feed compositions and splits, stream 28 is provided so that either $\mathrm{HNO}_{3}$ or $\mathrm{NaOH}$ may be added as needed. 
A comparison of the first iteration in the conceptual design report with the latest iteration reveals some information worth noting. The combination of streams 3 and 4 in the sludge dissolver was found to generate a significant amount of heat. This is not surprising since an acid-base neutralization is taking place there. A heat exchanger was placed after the sludge dissolver to cool stream 5 if necessary. Since earlier studies at Oak Ridge National Laboratory (Lloyd and Leuze 1961, Lloyd 1963) suggest improved anion exchange at higher temperatures, it may not be desirable to cool stream 5 . This would have to be verified during process optimization. Also worth noting is that the flow rate of each stream is nearly identical in both iterations. This is to be expected since most of the stream splits are based on defined separations.

The precipitation of sodium and cesium salts is not observed anywhere in the flow sheet, which is advantageous from a processing viewpoint. Hydrofluoric acid is observed to form in some streams, whereas hydrochloric acid is not observed in any of the streams. Trace amounts of nitrous acid are also observed in some streams. In many streams nitric acid is observed with free nitrate, although the concentration of free nitrate far exceeds that of the nitric acid.

\section{Conclusions and Recommendations}

This flow sheet model is particularly useful for performing "what if" and optimization analyses for the final design of a processing facility. However, since several assumptions were made in the development of the flowsheet, experiments should be performed on actual tank waste before final design decisions are made. The results of these experiments can then be incorporated into another iteration of the ASPEN Plus TM model. This would make the model even more realistic and useful as an optimization tool for the final design. 


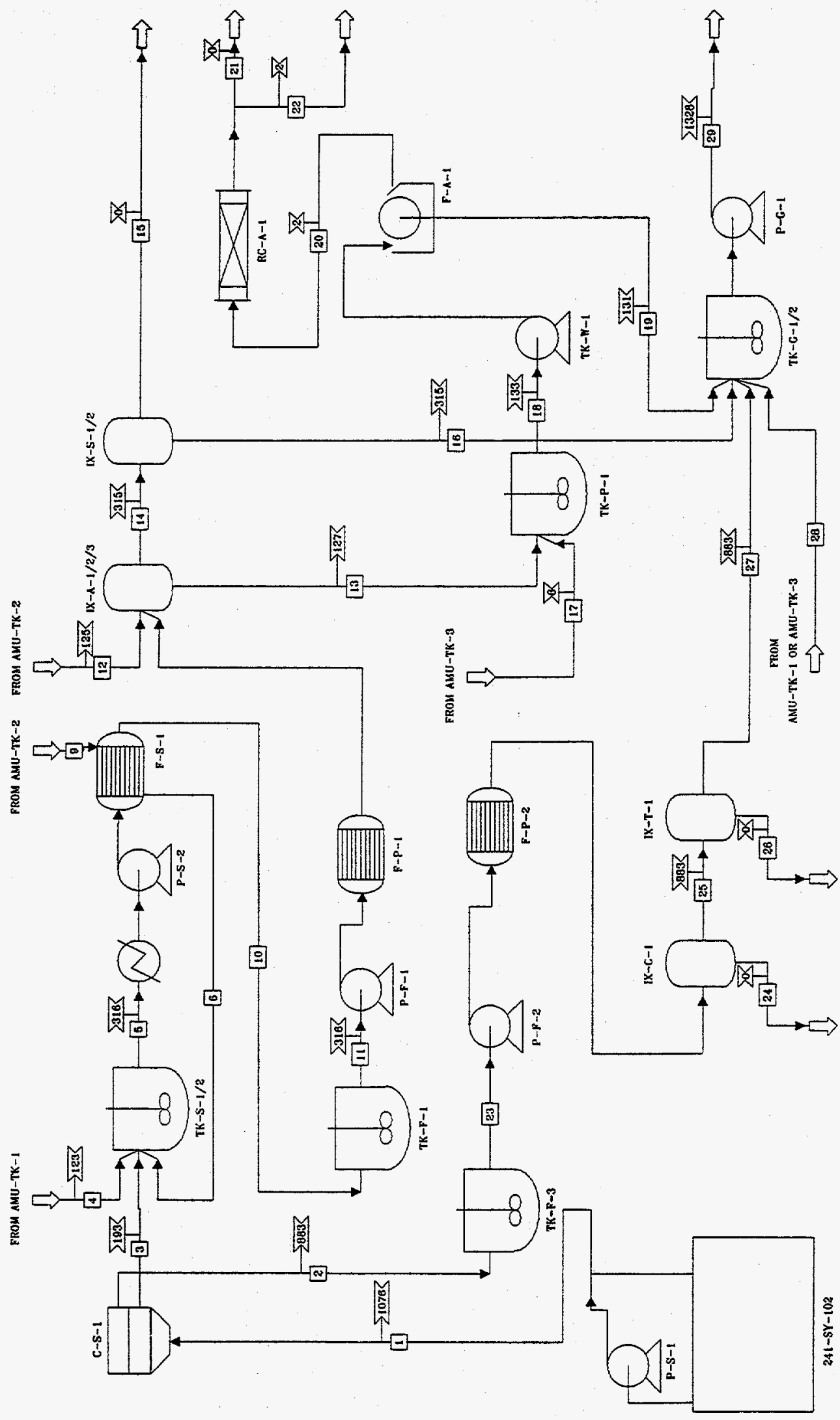

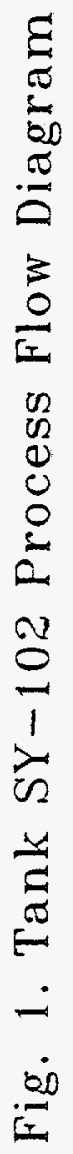




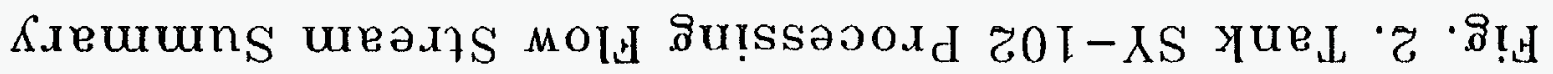

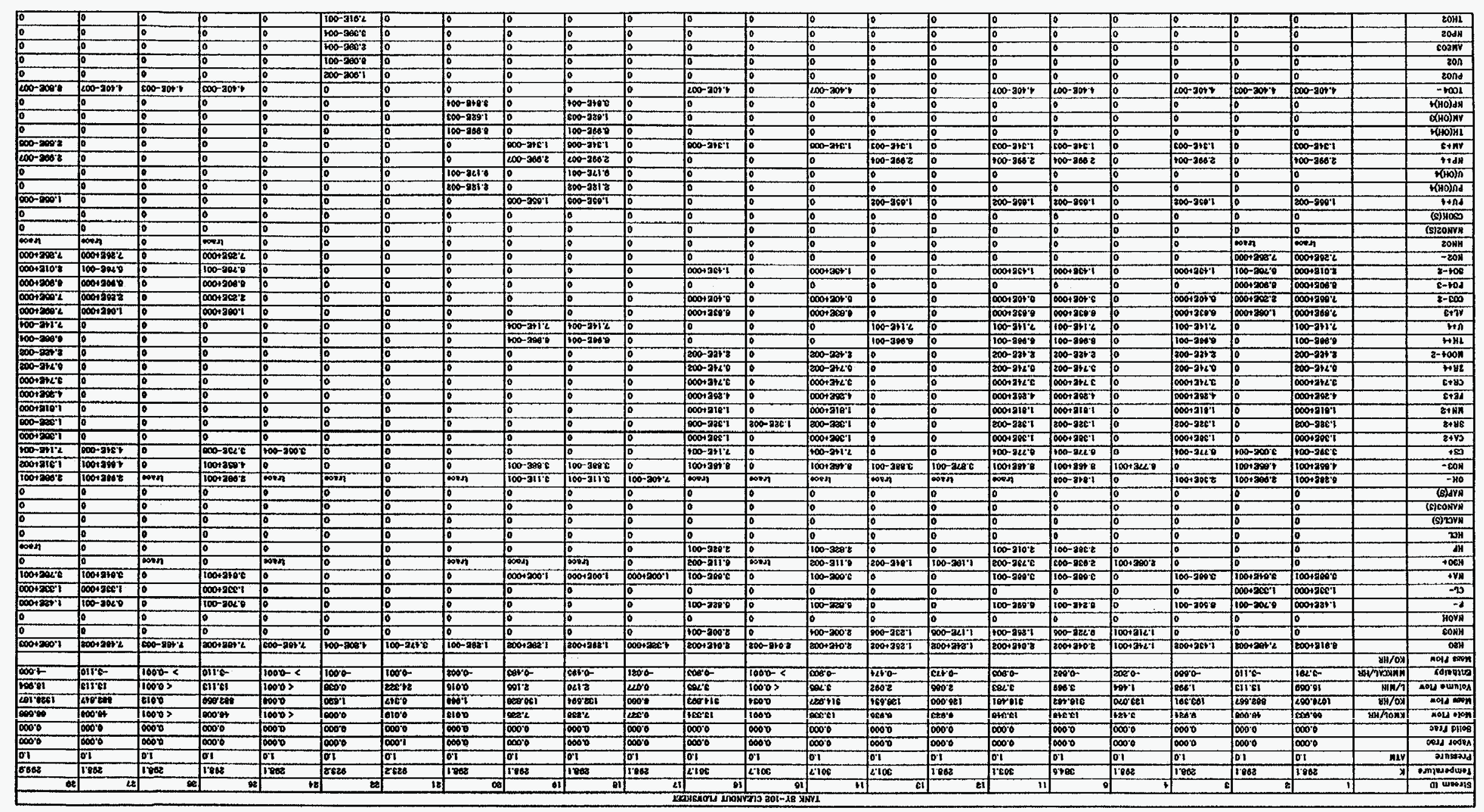




\section{Table 1 \\ Equipment Characteristics}

Item Designator Characteristics

241-SY-102 Description: Subterranean carbon steel tank lined with concrete, right cylinder with a domed top and a flat bottom

Capacity: $\quad 1.1 \times 10^{6}$ gallons $=147,000 \mathrm{ft}^{3}$

Size: $\quad$ Diameter $=75 \mathrm{ft}$, height $=35 \mathrm{ft}$

Contents: (As of 12/92) 2,551,000 L of slurry, with 396,000 L of sludge and $2,155,000 \mathrm{~L}$ of liquid

P-S-1 Description: Recirculation pump to wash and suspend the sludge as a slurry using an internal spray nozzle

Capacity: $\quad 106,000 \mathrm{~L} / \mathrm{hr}$ or $1,800 \mathrm{~L} / \mathrm{min}$

Basis: $\quad$ Turnover of the tank contents once per 24 hour period

C-S-1 Description: Centrifuge to separate solids from liquids in slurry

Capacity: $\quad 1,000 \mathrm{~L} / \mathrm{hr}$

Basis: $\quad$ Provide feed to sludge dissolver at $120 \mathrm{~L} / \mathrm{hr}(0.6-\mathrm{m}$ bowl diameter)

Size: $\quad$ Estimated at $1.5 \mathrm{~m} \mathrm{X} 1.5 \mathrm{~m} \mathrm{X} 2.0 \mathrm{~m}$

TK-S-1/2 Description: Two sludge dissolvers using a heated/stirred tanks to dissolve sludge in a batch mode, one vessel receiving feed from the centrifuge and the other dissolving and transferring feed

Capacity: $\quad 2,500 \mathrm{~L}$

Basis: $\quad$ Provide a dissolved acidic feed to the actinide column feed tank

Size: $\quad$ Cylindrical $\mathrm{D}=1.25 \mathrm{~m}, \mathrm{H}=2.0 \mathrm{~m}$

AMU-TK-1 Description: Aqueous Make-Up tank for concentrated nitric acid to feed TK-S-1/2

Capacity: $\quad 1,000 \mathrm{~L}$

Basis: $\quad$ Provide $700 \mathrm{~L}$ nitric acid per 8-hr shift

Size: $\quad$ Cylindrical $\mathrm{D}=0.8 \mathrm{~m}, \mathrm{H}=1.0 \mathrm{~m}$

P-S-2 Description: Solution pump to drive dissolved acidic feed through the slurry filter and into the feed tank

Capacity: $\quad 400 \mathrm{~L} / \mathrm{hr}$

Basis: Provide feed to sludge dissolver at $227 \mathrm{~L} / \mathrm{hr}$

F-S-1 Description: Slumy filter to remove residual sludge solids from the acidic feed

Capacity: $\quad 400 \mathrm{~L} / \mathrm{hr}$

Basis: Filters flushed with nitric acid back into a sludge dissolver tank

TK-F-1 Description: Feed tank

Capacity: $2,500 \mathrm{~L}$

Basis: Tank receiving feed from sludge dissolver and feeding the actinide column

Size: $\quad$ Cylindrical $D=1.25 \mathrm{~m}, \mathrm{H}=2.0 \mathrm{~m}$

P-F-1 Description: Feed pump to drive dissolved acidic feed through the polish filters and on through the actinide and strontium columns

Capacity: $\quad 400 \mathrm{~L} / \mathrm{hr}$

Basis: Maintain established volume velocities through the resin beds

F-P-1 Description: Polish filter to remove solids from the acidic feed to protect the resin beds

Capacity: $\quad 400 \mathrm{~L} / \mathrm{hr}$

Basis: $\quad$ Fine filters which can be backflushed with acid into the sludge dissolver 
IX-A-1/2/3 Description: Three actinide anion exchange columns for removal of thorium, uranium, plutonium, and americium (loaded with Reillex HPQ resin)

Capacity: $\quad 227 \mathrm{~L} / \mathrm{hr}$ on $500 \mathrm{~L}$ resin

Basis: $\quad$ One column on line, one column being eluted, one column in standby

Size: $\quad$ Cylindrical $\mathrm{D}=0.4 \mathrm{~m}, \mathrm{H}=4.0 \mathrm{~m}$

AMU-TK-2 Description: Aqueous make-up tank for dilute nitric acid to elute IX-A-1/2/3

Capacity: $\quad 1,500 \mathrm{~L}$

Basis: $\quad$ Provide $1,000 \mathrm{~L}$ solution per 8-hr shift

Size: $\quad$ Cylindrical $\mathrm{D}=0.8 \mathrm{~m}, \mathrm{H}=1.5 \mathrm{~m}$

IX-S-1/2 Description: Two strontium ion exchange columns for removal of strontium from acid solution (loaded with Sr Spec resin)

Capacity: $\quad 227 \mathrm{~L} / \mathrm{hr}$ on $125 \mathrm{~L}$

Basis: One column on line, one column in standby

Size: $\quad$ Cylindrical $\mathrm{D}=0.4 \mathrm{~m}, \mathrm{H}=1.0 \mathrm{~m}$

TK-P-1/2 Description: Two actinide precipitators

Capacity: $\quad 2,500 \mathrm{~L}$

Basis: One tank receiving effluent from the actinide column, the other precipitating actinide hydroxides and feeding the actinide slurry filter

Size: $\quad$ Cylindrical $D=1.25 \mathrm{~m}, \mathrm{H}=2.0 \mathrm{~m}$

P-A-1 Description: Actinide slurry pump to feed the slurry filter

Capacity: $250 \mathrm{~L} / \mathrm{hr}$

Basis: $\quad 100 \mathrm{~L} / \mathrm{hr}$ precipitate and $130 \mathrm{~L} / \mathrm{hr}$ filtrate

F-A-1 Description: Actinide slurry filter to remove actinide hydroxide precipitate from solution with the filtrate going on to the grout neutralization tank and the cake on to the actinide rotary calciner

Capacity: $\quad 250 \mathrm{~L} / \mathrm{hr}$

Basis: Drum filter sized to remove hydroxide cake in a continuous manner $\left(2.8 \mathrm{~m}^{2}\right.$ of filter)

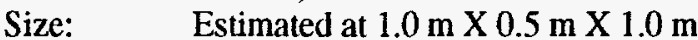

RC-A-1 Description: Actinide rotary calciner to convert the actinide hydroxide cake to oxide

Capacity: $2 \mathrm{~L} / \mathrm{hr}$

Basis: Calciner sized to convert the hydroxide cake to oxide in a continuous manner

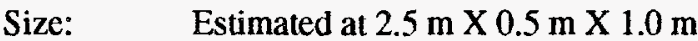

TK-F-2 Description: Feed tank

Capacity: $\quad 10,000 \mathrm{~L}$

Basis: Tank receiving feed from the centrifuge and feeding the cesium and technetium columns

Size: $\quad$ Cylindrical $\mathrm{D}=2 \mathrm{~m}, \mathrm{H}=3.0 \mathrm{~m}$

P-F-2 Description: Feed pump to drive basic feed through the polish filters and on through the cesium and technetium columns

Capacity: $\quad 1,000 \mathrm{~L} / \mathrm{hr}$

Basis: $\quad$ Maintain established volume velocities through the resin beds

F-P-2 Description: Polish filter to remove solids from the basic feed to protect the resin beds

Capacity: $\quad 1,000 \mathrm{~L} / \mathrm{hr}$

Basis: $\quad$ Fine filters which can be backflushed with acid into the sludge dissolver 
IX-C-1 Description: Cesium ion exchange columns for removal of cesium from basic solution

Capacity: $\quad 781 \mathrm{~L} / \mathrm{hr}$, loading capacity limited by thermal loading

Basis: $\quad$ One column on line, resin changed when breakthrough achieved

Size: $\quad$ Cylindrical $\mathrm{D}=0.75 \mathrm{~m}, \mathrm{H}=2.0 \mathrm{~m}$

IX-T-1 Description: Technetium anion exchange columns for removal of technetium from basic solution

Capacity: $\quad 781 \mathrm{~L} / \mathrm{hr}$

Basis: $\quad$ One column on line, resin changed when breakthrough achieved

Size: $\quad$ Cylindrical $\mathrm{D}=0.75 \mathrm{~m}, \mathrm{H}=1.0 \mathrm{~m}$

TK-G-1/2 Description: Two grout neutralization tanks

Capacity: $\quad 15,000 \mathrm{~L}$

Basis: One tank receiving effluent from the strontium and technetium columns as well as the actinide slurry filtrate, the other neutralizing the waste in preparation for grouting.

Size: $\quad$ Cylindrical $\mathrm{D}=2.2 \mathrm{~m}, \mathrm{H}=4.0 \mathrm{~m}$

AMU-TK-3 Description: Aqueous make-up tank for sodium hydroxide to feed TK-P-1 and TK-G$1 / 2$.

Capacity: $\quad 1,000 \mathrm{~L}$

Basis: $\quad$ Provide up to $700 \mathrm{~L}$ sodium hydroxide per 8-hr shift

Size: $\quad$ Cylindrical $\mathrm{D}=0.8 \mathrm{~m}, \mathrm{H}=1.0 \mathrm{~m}$

P-G-1 Description: Grout slurry pump to transfer neutralized solutions to the grout facilities Capacity: $\quad 1,500 \mathrm{~L} / \mathrm{hr}$

Basis: Empty tank to transfer vehicle and subsequent low level waste storage area in a timely manner 


\section{References}

Lumetta, G. J. and J. L. Swanson, Pretreatment of Plutonium Plant (PFP) Sludge: Report for the Period October 1990 - March 1992, TWRSPP-92-011 Draft (March 9, 1992).

Lloyd, M. H., Nuclear Science and Engineering, 17, 452 (1963).

Lloyd, M. H. and R. E. Leuze, Nuclear Science and Engineering, 11, 274 (1961).

Mostafa, A. T. M. G., J. M. Eakman, and S. L. Yarbro, submitted to Industrial and Engineering Chemistry (January 1995).

Punjak, W. A., S. L. Yarbro, S. B. Schreiber, S. L. Dunn, E. M. Ortiz, R. S. Day, and K. W. Thomas, Scale-up of the SY-102 Remediation Flowsheet, Los Alamos National Laboratory report LA-12793-MS (August 1994).

Yarbro, S. L., W. A. Punjak, S. B. Schreiber, S. L. Dunn, G. D. Jarvinen, S. F. Marsh, S. Agnew, E. Birnbaum, and K. W. Thomas, Status of Tank 102-SY Remediation Flowsheet Development at LANL, Los Alamos National Laboratory document LA-UR-12706 (February 1994).

Yarbro, S. L., W. A. Punjak, S. B. Schreiber, S. L. Dunn, G. D. Jarvinen, S. F. Marsh, N. G. Pope, S. Agnew, E. R. Birnbaum, K. W. Thomas, and E. A. Ortic, Tank SY-102 Remediation Project - Flowsheet and Conceptual Design Report, Los Alamos National Laboratory report LA-12701-MS (January 1994). 


\section{APPENDIX}


; Input Summary created by ASPEN PLUS Rel. 9.1-3 on 11:03:47 Fri Feb 3, 1995 ; Directory C: \SY102 Filename C: \SY102\sy102q.inp

;

TITLE 'TANK SY-102 CLEANOUT FLOWSHEET'

IN-UNTTS MET

DEF-STREAMS CONVEN ALL

DATABANKS ASPENPCD / AQUEOUS $/$ SOLIDS $/$ INORGANIC $/ \&$ PURECOMP

PROP-SOURCES ASPENPCD
PURECOMP

\section{COMPONENTS}

$\mathrm{H} 2 \mathrm{O} \mathrm{H} 2 \mathrm{O} \mathrm{H} 2 \mathrm{O} /$

HNO3 HNO3 HNO3/

$\mathrm{NAOH} \mathrm{NAOH} \mathrm{NAOH} /$

F- F- F- /

$\mathrm{CL}-\mathrm{CL}-\mathrm{CL}-/$

NAt NAt NAt $/$

$\mathrm{H} 3 \mathrm{O}+\mathrm{H} 3 \mathrm{O}+\mathrm{H} 3 \mathrm{O}+/$

$\mathrm{HF} \mathrm{HF} \mathrm{HF} /$

HCL HCL HCL /

"NACL(S)" NACL "NACL(S)" /

"NANO3 (S)" NANO3 "NANO3 (S)" /

"NAF (S)" NAF "NAF (S)" $/$

$\mathrm{OH}-\mathrm{OH}-\mathrm{OH}-/$

NO3 - NO3 - NO3- $/$

$\mathrm{CS}+\mathrm{CS}+\mathrm{CS}+1$

$\mathrm{CA}+2 \mathrm{CA}+2 \mathrm{CA}+2 /$

$\mathrm{SR}+2 \mathrm{SR}+2 \mathrm{SR}+2 /$

$\mathrm{MN}+2 \mathrm{MN}+2 \mathrm{MN}+2 /$

$\mathrm{FE}+3 \mathrm{FE}+3 \mathrm{FE}+3$ /

$\mathrm{CR}+3 \mathrm{CR}+3 \mathrm{CR}+3 /$

$\mathrm{ZR}+4 \mathrm{ZR}+4 \mathrm{ZR}+4 /$

MOO4-2 MOO4-2 MOO4-2 /

$\mathrm{TH}+4 \mathrm{TH}+4 \mathrm{TH}+4 \quad /$

$\mathrm{U}+4 \quad \mathrm{U}+4 \mathrm{U}+4$ /

$\mathrm{AL}+3 \mathrm{AL}+3 \mathrm{AL}+3$ /

CO3-2 $\mathrm{CO} 3-2 \mathrm{CO}-2 /$

PO4-3 PO4-3 PO4-3/

SO4-2 SO4-2 SO4-2/

NO2 - NO2- NO2- $/$

$\mathrm{HNO}_{2} \mathrm{HNO}_{2} \mathrm{HNO}_{2} /$

"NANO2 (S)" NANO2 "NANO2 (S)" /

"CSOH (S)" $\mathrm{CSOH}$ "CSOH (S)" /

$\mathrm{PU}+4$ * $\mathrm{PU}+4$ /

"PU (OH) 4 " * "PU (OH) 4 " /

"U(OH) 4" "U(OH) 4" "U(OH) 4" /

$\mathrm{NP}+4$ * $\mathrm{NP}+4 /$

$\mathrm{AM}+3 * \mathrm{AM}+3 /$

"TH $(\mathrm{OH}) 4 "$ * "TH(OH) $4 " /$

"AM(OH) 3" * "AM(OH) 3"/

"NP(OH) 4" * "NP(OH) 4" / 


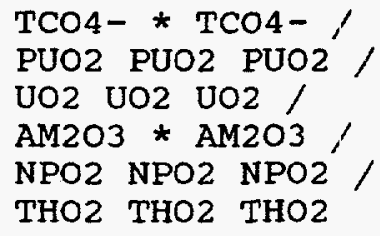

HENRY-COMPS GLOBAL HCL

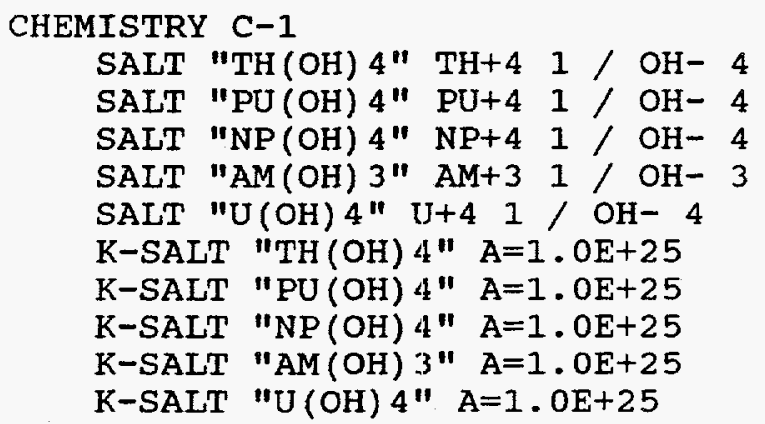

CHEMISTRY GLOBAL

IN-UNITS MET VOLUME-FLOW=' CUM/HR' ENTHALPY-FLO='MMKCAL/HR' \& HEAT-TRANS-- $\mathrm{C}={ }^{\prime} \mathrm{KCAL} / \mathrm{HR}-\mathrm{SQM}-\mathrm{K}^{\prime}$ PRESSURE=BAR TEMPERATURE $=\mathrm{C} \&$ VOLUME=CUM DELTA $-T=C$ HEAD=METER MOLE-DENSITY $=\prime$ KMOL/CUM' \& MASS-DENSITY $=$ ' KG/CUM' MOLE-ENTHALP=' KCAL/MOL' \& MASS-ENTHAIJP='KCAL/KG' HEAT=MMKCAL MOLE-CONC $=\prime M O L / L^{\prime} \&$ $P D R O P=B A R$

DISS NAOH NAT $\mathrm{L} / \mathrm{OH}-1$

STOIC $1 \mathrm{H} 2 \mathrm{O}-2 / \mathrm{H} 3 \mathrm{O}+1 / \mathrm{OH}-1$

STOIC $2 \mathrm{HCL}-1 / \mathrm{H} 2 \mathrm{O}-1 / \mathrm{H} 3 \mathrm{O}+1 / \mathrm{CL}-1$

STOIC $3 \mathrm{HF}-1 / \mathrm{H} 2 \mathrm{O}-1 / \mathrm{H} 3 \mathrm{O}+1 / \mathrm{F}-1$

STOIC 4 HNO3 - $1 / \mathrm{H}_{2} \mathrm{O}-1 / \mathrm{H} 3 \mathrm{O}+1 / \mathrm{NO} 3-1$

STOIC 5 HNO2 - $1 / \mathrm{H} 2 \mathrm{O}-1 / \mathrm{H} 3 \mathrm{O}+1 / \mathrm{NO} 2-1$

$\mathrm{K}$-STOIC $1 \mathrm{~A}=132.89888 \mathrm{~B}=-13445.9 \mathrm{C}=-22.4773 \mathrm{D}=0$

$\mathrm{K}$-STOIC $5 \mathrm{~A}=-11.592564 \quad \mathrm{~B}=0 \quad \mathrm{C}=0 \quad \mathrm{D}=0$

SALT "NACL(S)" NA+ $1 / \mathrm{CL}-1$

SALT "CSOH(S)" $\mathrm{CS}+1 / \mathrm{OH}-1$

SALT "NANO2(S)" NA+ $1 / \mathrm{NO2}-1$

SALT "NANO3 (S)" NAt $1 /$ NO3- 1

K-SALT "NACL(S)" $A=46.59531 \quad B=-4226.687 \quad C=-5.642413$ \& $D=-.01699288$

\section{FLOWSHEET}

BLOCK C-S-1 IN=1 OUT $=2 \quad 3$

BLOCK TK-S-1 IN $=4 \quad 3$ OUT $=5$

BLOCK IX-A-1 IN=11 12 OUT=13 14

BLOCK IX-S-1 IN=14 OUT=15 16

BLOCK IX-C-1 IN=2 OUT $=25 \quad 24$

BLOCK IX-T-1 IN=25 OUT=26 27

BLOCK TK-P-1 IN $=13 \quad 17 \quad$ OUT $=18$

BLOCK $\quad \mathrm{F}-\mathrm{A}-1 \quad \mathrm{IN}=18$ OUT $=1920$

BLOCK TK-G-1 IN=19 $27 \quad 16$ OUT=29

BLOCK $X-S-1 \quad I N=5$ OUT $=11$

BLOCK RC $-\mathrm{A}-1$ IN $=20$ OUT $=30$

BLOCK SPLIT IN=30 OUT $=2122$

PROPERTIES ELECNRTI HENRY-COMPS=GLOBAL CHEMISTRY=GLOBAL \& TRUE-COMPS=YES 


\section{ESTIMATE ALL}

PROP-DATA U-2

IN-UNITS SI

PROP-LIST TC / PC / ZC / RKTZRA / VC / VCRKT

PVAL HNO2 $520.000 / 6.89010 E+06 / 0.231000 / 0.221008 /$ \& $0.145000 / 0.145000$

PROP-LIST TC / PC / ZC / VC

PVAL UO2 $507.400 / 2.96882 \mathrm{E}+06 / 0.260 / 0.369445$

PVAL PUO2 $507.400 / 2.96882 \mathrm{E}+06 / 0.260 / 0.369445$

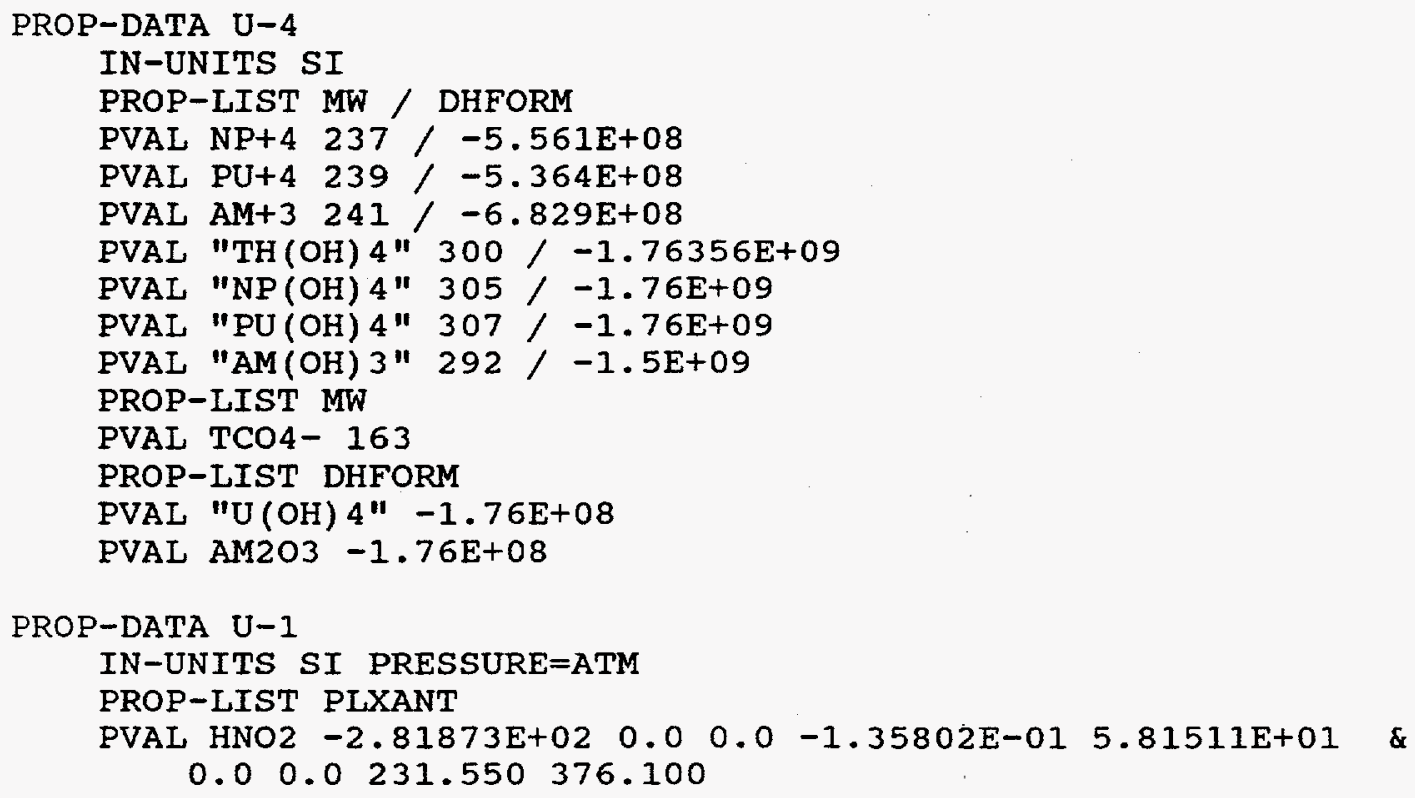




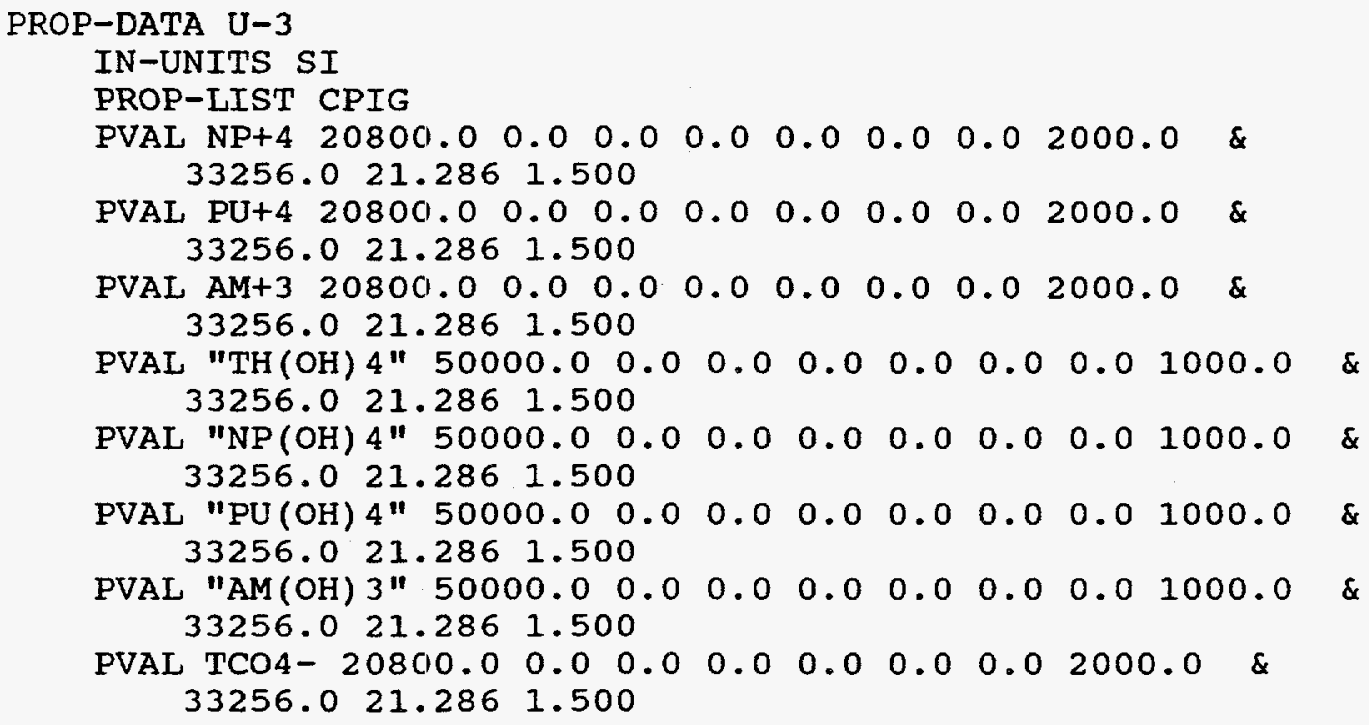

PROP-DATA NRTL-1

IN-UNITS MET VOLUME-FLOW='CUM/HR' ENTHALPY-FLO='MMKCAL/HR' \& HEAT-TRANS-C $=\prime \mathrm{KCAL} / \mathrm{HR}-\mathrm{SQM}-\mathrm{K}^{\prime}$ PRESSURE=BAR TEMPERATURE $=\mathrm{C}$ \& VOLUME $=C U M$ DELTA $-\mathrm{T}=\mathrm{C}$ HEAD=METER MOLE-DENSITY $=$ 'KMOL/CUM' \& MASS-DENSIT'Y='KG/CUM' MOLE-ENTHALP='KCAL/MOL' \& MASS-ENTHAL,P=' $\mathrm{KCAL} / \mathrm{KG}{ }^{\prime} \mathrm{HEAT}=\mathrm{MMKCAL} \mathrm{MOLE}-\mathrm{CONC}={ }^{\prime} \mathrm{MOL} / \mathrm{L}$ ' \& PDROP=BAR

PROP-LIST NRTL

BPVAL H2O HF $97.28083 \quad 0.0 \quad .3000000 \quad 0.0 \quad 0.0 \quad 0.0 \quad 25.00000 \quad \&$ 110.0000

BPVAL HF H2O $-2.297253 \quad 0.0 \quad .3000000 \quad 0.0 \quad 0.0 \quad 0.025 .00000$ \& 110.0000

PROP-DATA VLCLK- 1

IN-UNITS MET VOLUME-FLOW=' CUM/HR' ENTHALPY-FLO='MMKCAL/HR' \& HEAT-TRANS-C $=$ ' $\mathrm{KCAL} / \mathrm{HR}-\mathrm{SQM}-\mathrm{K}$ ' PRESSURE $=\mathrm{BAR}$ TEMPERATURE $=\mathrm{C}$ \& VOLUME=CUM DELTA-T'=C HEAD=METER MOLE-DENSITY='KMOL/CUM' \& MASS-DENSITY='KG/CUM' MOLE-ENTHALP='KCAL/MOI' \& MASS-ENTHALP=' KCAL/KG' HEAT=MMKCAL MOLE-CONC='MOL/L' \& PDROP=BAR

PROP-LIST VLCLK:

BPVAL CL- NA+ 15.14218 25.28718

BPVAL CL- H3O+ 34.55111 13.36581

BPVAL NA+ OH - - 13.7942072 .92090

BPVAL H3O+ NO3-38.26718 38.32753

BPVAL NAt SO4-2 8.675526123 .2671

PROP-DATA GMELCC-1

IN-UNITS MET VOLUME-FLOW='CUM/HR' ENTHALPY-FLO='MMKCAL/HR' \& 
HEAT-TRANS-C='KCAL/HR-SQM-K' PRESSURE=BAR TEMPERATURE=C VOLUME $=C U M$ DELTA-T $=C$ HEAD=METER MOLE-DENSITY $=$ ' KMOL/CUM' MASS-DENSITY $=$ ' KG/CUM' MOLE-ENTHALP $=$ ' $\mathrm{KCAL} / \mathrm{MOL}$ ' \&

MASS-ENTHALP $=$ ' $\mathrm{KCAL} / \mathrm{KG}{ }^{\prime} \mathrm{HEAT}=\mathrm{MMKCAL} \mathrm{MOLE}-\mathrm{CONC}={ }^{\prime} \mathrm{MOL} / \mathrm{L}{ }^{\prime}$ \& PDROP $=$ BAR

PROP-IIST GMELCC

PPVAL H2O ( NA+ CL- ) 5.980196

PPVAL ( NA+ CL- ) H2O -3.789168

PPVAL H2O ( NAt OH- ) 6.738000

PPVAL ( NAt OH- ) H2O -3.771221

PPVAL H2O ( NAt NO3-) 7.167000

PPVAL ( NA+ NO3-) H2O - 3.645000

PPVAL H2O ( H3O+ F- ) 15.12827

PPVAL ( $\mathrm{H} 3 \mathrm{O}+\mathrm{F}-$ ) $\mathrm{H} 2 \mathrm{O}-2.348784$

PPVAL H2O ( $\mathrm{H} 3 \mathrm{O}+\mathrm{CL}-$ ) 4.110129

PPVAL ( $\mathrm{H} 3 \mathrm{O}+\mathrm{CL}-$ ) H2O -3.344103

PPVAL H2O ( $\mathrm{H} 3 \mathrm{O}+\mathrm{OH}^{-}$) 8.045000

PPVAL ( $\mathrm{H} 3 \mathrm{O}+\mathrm{OH}-$ ) $\mathrm{H} 2 \mathrm{O}-4.072000$

PPVAL H2O ( $\mathrm{H} 3 \mathrm{O}+\mathrm{NO} 3-) 5.853772$

PPVAL ( $\mathrm{H} 3 \mathrm{O}+\mathrm{NO} 3-$ ) $\mathrm{H} 2 \mathrm{O}-2.858350$

PPVAL HNO3 ( $\mathrm{H} 3 \mathrm{O}+\mathrm{NO3-}$ ) -10.05384

PPVAL ( $\mathrm{H} 3 \mathrm{O}+\mathrm{NO3}-$ ) HNO3 -4.616377

PPVAL ( NAt CL- ) HCL -8.000000

PPVAL HCL ( NA+ CL- ) 15.00000

PPVAL ( NA+ OH-) HCL -8.000000

PPVAL HCL ( NA+ OH- ) 15.00000

PPVAL ( $\mathrm{H} 3 \mathrm{O}+\mathrm{F}-$ ) HF 3.566598

PPVAL HF ( H3O+ F-) 9.667216

PPVAL ( $\mathrm{H} 3 \mathrm{O}+\mathrm{CL}-$ ) HCL $-1.0000 \mathrm{E}-3$

PPVAL HCL ( $\mathrm{H} 3 \mathrm{O}+\mathrm{CL}-$ ) 12.00000

PPVAL ( $\mathrm{H} 3 \mathrm{O}+\mathrm{OH}-$ ) HCL -8.000000

PPVAL HCL ( H3O+ OH- ) 15.00000

PPVAL H2O ( $\mathrm{CA}+2 \mathrm{CL}-$ ) 10.47200

PPVAL ( $\mathrm{CA}+2 \mathrm{CL}-) \mathrm{H} 2 \mathrm{O}-5.060000$

PPVAL H2O ( $\mathrm{CA}+2 \mathrm{NO}-$ ) 7.578000

PPVAL ( $\mathrm{CA}+2 \mathrm{NO}-$ ) H2O -4.072000

PPVAL H2O ( $\mathrm{SR}+2 \mathrm{CL}-$ ) 9.634000

PPVAL ( SR+2 CL- ) H2O -4.819000

PPVAL H2O ( SR+2 NO3-) 7.618000

PPVAL ( SR+2 NO3-) H2O -3.894000

PPVAL H2O ( $\mathrm{MN}+2 \mathrm{CL}$ ) ) 8.341000

PPVAL ( $\mathrm{MN}+2 \mathrm{CL}-$ ) H2O -4.276000

PPVAL H2O (NAt CO3-2) -4.833000

PPVAL ( NAt CO3-2) H2O .9770000

PPVAL H2O ( NA+ SO4-2) 7.689221

PPVAL ( NAt SO4-2) H2O -4.284786

PPVAL H2O ( $\left.\mathrm{H} 30+\mathrm{CO}_{3}-2\right) 8.045000$

PPVAL ( $\mathrm{H} 3 \mathrm{O}+\mathrm{CO} 3-2$ ) $\mathrm{H} 2 \mathrm{O}-4.072000$

PPVAL H2O ( $\mathrm{H} 3 \mathrm{O}+\mathrm{SO} 4-2$ ) 8.000000

PPVAL ( $\mathrm{H} 3 \mathrm{O}+\mathrm{SO} 4-2$ ) H2O -4.000000

PPVAL ( $\mathrm{H} 3 \mathrm{O}+\mathrm{SO} 4-2) \mathrm{HCL}-8.000000$

PPVAL HCL ( H3O+ SO4-2) 15.00000

PPVAL H2O ( NA+ NO2- ) 7.539000

PPVAL ( NAt NO2-) H2O -3.889000

PROP-DATA GMELCD-1

IN-UNITS MET VOLUME-FLOW='CUM/HR' ENTHALPY-FLO='MMKCAL/HR' \& HEAT-TRANS- $C=$ ' $\mathrm{KCAL} / \mathrm{HR}-\mathrm{SQM}-\mathrm{K}$ ' PRESSURE $=\mathrm{BAR}$ TEMPERATURE $=\mathrm{C} \&$ VOLUME $=C U M$ DELTA-T $=C$ HEAD $=$ METER MOLE-DENSITY $=$ ' KMOL $/ C U M \prime \&$ MASS-DENSITY $=$ 'KG/CUM' MOLE-ENTHALP $=$ ' $\mathrm{KCAL} / \mathrm{MOL} \mathrm{L}^{\prime} \quad \&$ 
MASS-ENTHAI_P $=$ ' $\mathrm{KCAL} / \mathrm{KG}$ ' HEAT $=\mathrm{MMKCAL} \mathrm{MOLE}-\mathrm{CONC}={ }^{\prime} \mathrm{MOL} / \mathrm{L}^{\prime}$ \& PDROP=BAR

PROP-LIST GMELCD

PPVAL H2O ( NAt CL- ) 841.5181

PPVAL ( NAt CL-- ) H2O -216.3646

PPVAL H2O ( NAt OH-) 1420.242

PPVAL ( NA+ OH-- ) H2O -471.8202

PPVAL H2O ( $\mathrm{H} 3 \mathrm{O}+\mathrm{F}-)-2141.079$

PPVAL ( $\mathrm{H} 3 \mathrm{O}+\mathrm{F}-\mathrm{H}^{-}$) $\mathrm{H} 2 \mathrm{O}-155.0825$

PPVAL H2O ( $\mathrm{H} 3 \mathrm{O}+\mathrm{CL}-$ ) 2306.642

PPVAL ( $\mathrm{H} 3 \mathrm{O}+\mathrm{Cl}-$ ) $\mathrm{H} 2 \mathrm{O}-653.5391$

PPVAL H2O ( $\mathrm{H} 30+\mathrm{NO} 3-$ ) 457.3743

PPVAL ( $\mathrm{H} 3 \mathrm{O}+\mathrm{NO} 3-$ ) $\mathrm{H} 2 \mathrm{O}-332.1241$

PPVAL HNO3 ( $\mathrm{H} 3 \mathrm{O}+\mathrm{NO3}-$ ) 5539.853

PPVAL ( $\mathrm{H} 3 \mathrm{O}+\mathrm{NO} 3-$ ) $\mathrm{HNO} 3242.2092$

PPVAL ( NA+ CL-- ) HCL 0.0

PPVAL HCL ( NAt CL-) 0.0

PPVAL ( NA+ OH-- ) HCL 0.0

PPVAL HCL ( NAt- OH- ) 0.0

PPVAL ( $\mathrm{H} 3 \mathrm{O}+\mathrm{F}-\mathrm{\text {) }} \mathrm{HF}-2071.534$

PPVAL HF ( $\mathrm{H} 3 \mathrm{O}+\mathrm{F}-$ ) -3161.984

PPVAL ( $\mathrm{H} 3 \mathrm{O}+\mathrm{Cl}-$ ) HCL 0.0

PPVAL HCL ( $\mathrm{H} 3 \mathrm{O}+\mathrm{CL}-) 0.0$

PPVAL ( $\mathrm{H} 3 \mathrm{O}+\mathrm{OH}^{-}$) $\mathrm{HCL} 0.0$

PPVAL HCL ( $\mathrm{H} 3 \mathrm{O}+\mathrm{OH}-) 0.0$

PPVAL H2O ( NAt $\mathrm{CO} 3-2$ ) 4018.400

PPVAL ( NAt CO3-2) H2O -1547.000

PPVAL H2O ( NAt SO4-2 ) 565.5983

PPVAL ( NAt SO4-2) H2O -56.83768

PPVAI H2O ( $\left.\mathrm{H} 3 \mathrm{O}+\mathrm{SO}_{4-2}\right) 0.0$

PPVAL ( $\mathrm{H} 3 \mathrm{O}+\mathrm{SO} 4-2$ ) $\mathrm{H} 2 \mathrm{O} 0.0$

PPVAL ( $\mathrm{H} 3 \mathrm{O}+\mathrm{SO} 4-2$ ) HCL 0.0

PPVAL HCL ( $\mathrm{H} 3 \mathrm{O}+\mathrm{SO} 4-2) 0.0$

PROP-DATA GMELCE-1

IN-UNITS MET VOLUME-FLOW=' CUM/HR' ENTHALPY-FLO='MMKCAL/HR' \& HEAT-TRANS-- $\mathrm{C}=$ ' $\mathrm{KCAL} / \mathrm{HR}-\mathrm{SQM}-\mathrm{K}$ ' PRESSURE=BAR TEMPERATURE $=\mathrm{C}$ \& VOLUME=CUM DELTA-T=C HEAD=METER MOLE-DENSITY $=$ 'KMOL/CUM' \& MASS-DENSIIIY $=$ 'KG/CUM' MOLE-ENTHALP='KCAL/MOL' \&

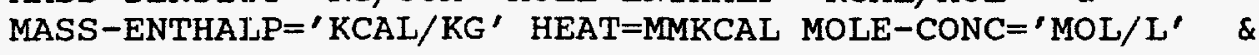
PDROP=BAR

PROP-IIST GMELCE

PPVAL H2O ( NA+ CL- ) 7.433500

PPVAL ( NA+ CL-- ) H2O -1.100418

PPVAL H2O ( NA+ OH- ) 3.013932

PPVAL ( NAt OH-- ) H2O 2.136557

PPVAL H2O ( $\mathrm{H} 3 \mathrm{O}+\mathrm{F}-$ ) .6771824

PPVAL ( $\mathrm{H} 3 \mathrm{O}+\mathrm{F}--$ ) $\mathrm{H} 2 \mathrm{O}-3.456933$

PPVAL H2O ( $\mathrm{H} 3 \mathrm{O}+\mathrm{CL}-$ ) .3417959

PPVAL ( $\mathrm{H} 3 \mathrm{O}+\mathrm{Cl}-$ ) $\mathrm{H} 2 \mathrm{O} 2.121453$

PPVAL H2O ( $\mathrm{H} 3 \mathrm{O}+\mathrm{NO} 3-) 0.0$

PPVAL ( $\mathrm{H} 3 \mathrm{O}+\mathrm{NO} 3-$ ) $\mathrm{H} 2 \mathrm{O} 0.0$

PPVAL HNO3 ( $\mathrm{H} 3 \mathrm{O}+\mathrm{NO3}-$ ) 100.0000

PPVAL ( $\mathrm{H} 3 \mathrm{O}+\mathrm{NO} 3-$ ) HNO3 33.37622

PPVAL ( NA+ CL- ) HCL 0.0

PPVAL HCL ( NA+ CL- ) 0.0

PPVAL ( NA+ OH- ) HCL 0.0

PPVAL HCL ( NA+ OH- ) 0.0

PPVAL ( $\mathrm{H} 3 \mathrm{O}+\mathrm{Cl}-$ ) HCL 0.0

PPVAL HCL ( $\mathrm{H} 3 \mathrm{O}+\mathrm{CL}-$ ) 0.0 
PPVAL ( $\mathrm{H} 3 \mathrm{O}+\mathrm{OH}-$ ) HCL 0.0

PPVAL HCL ( $\mathrm{H} 3 \mathrm{O}+\mathrm{OH}-$ ) 0.0

PPVAL H2O ( $\mathrm{NA}+\mathrm{CO} 3-2$ ) 88.56000

PPVAL ( NA+ $\mathrm{CO} 3-2$ ) $\mathrm{H} 2 \mathrm{O}-32.40000$

PPVAL H2O ( NA+ SO4-2) -14.08276

PPVAL ( NAT SO4-2) H2O 8.547499

PPVAL ( $\mathrm{H} 3 \mathrm{O}+\mathrm{SO} 4-2$ ) HCL 0.0

PPVAL HCL ( $\mathrm{H} 3 \mathrm{O}+\mathrm{SO}-2$ ) 0.0

PROP-DATA GMELCN-I

IN-UNITS MET VOLUME-FLOW=' CUM/HR' ENTHALPY-FLO='MMKCAL/HR' \& HEAT-TRANS $-\mathrm{C}=$ ' $\mathrm{KCAL} / \mathrm{HR}-\mathrm{SQM}-\mathrm{K}$ ' PRESSURE=BAR TEMPERATURE $=\mathrm{C} \quad$ \& VOLUME=CUM DELTA $-\mathrm{T}=\mathrm{C}$ HEAD=METER MOLE-DENSITY $=$ 'KMOL/CUM' \& MASS-DENSITY $=$ ' $\mathrm{KG} / \mathrm{CUM}$ ' MOLE-ENTHALP $=$ ' $\mathrm{KCAL} / \mathrm{MOL}$ ' \& MASS -ENTHALP $=$ ' $\mathrm{KCAL} / \mathrm{KG}$ ' HEAT $=\mathrm{MMKCAL} \mathrm{MOLE}-\mathrm{CONC}=' \mathrm{MOL} / \mathrm{L}$ ' \& PDROP=BAR

PROP-LIST GMELCN

PPVAL H2O ( NA+ CL- ) .2000000

PPVAL H2O ( NA+ OH-) .2000000

PPVAL H2O ( $\mathrm{H} 3 \mathrm{O}+\mathrm{F}-$ ) .2000000

PPVAL ( NAT CL- ) HCL . 1000000

PPVAL ( NA+ OH- ) HCL. 1000000

PPVAL ( $\mathrm{H} 3 \mathrm{O}+\mathrm{OH}-$ ) $\mathrm{HCL} .1000000$

PPVAL H2O ( NAt $\mathrm{CO} 3-2$ ) .2000000

PPVAL H2O ( NAt SO4-2) .2000000

PPVAL ( $\mathrm{H} 30+\mathrm{SO} 4-2$ ) HCL . 1000000

STREAM 1

SUBSTREAM MIXED TEMP $=25.0<\mathrm{C}>$ PRES $=1.0$ NPHASE $=2$

MASS-FLOW H2O $891 / \mathrm{F}-1.42 / \mathrm{CL}-1.33 / \mathrm{NA}+36.8 / \&$ $\mathrm{H} 3 \mathrm{O}+0.0 / \mathrm{OH}-52.8 / \mathrm{NO} 3-46.5 / \mathrm{CS}+3.39 \mathrm{E}-4 / \&$

$\mathrm{CA}+21.38 / \mathrm{SR}+21.31 \mathrm{E}-2 / \mathrm{MN}+21.81 / \mathrm{FE}+34.25 / \&$

$\mathrm{CR}+33.74 / \mathrm{ZR}+45.74 \mathrm{E}-2$ / $\mathrm{MOO} 4-22.40 \mathrm{E}-2 / \mathrm{TH}+4$ \&

$0.696 / \mathrm{U}+40.714 / \mathrm{AL}+37.69 / \mathrm{CO3-2} 7.65 / \&$

$\mathrm{PO} 4-38.90 / \mathrm{SO} 4-22.01 / \mathrm{NO} 2-7.25 / \mathrm{PU}+4 \&$

$1.65 \mathrm{E}-02 / \mathrm{NP}+42.99 \mathrm{E}-04 / \mathrm{AM}+3 \mathrm{i}+34 \mathrm{E}-03 / \mathrm{TCO} 4-\&$

$4.40 E-03$

STREAM 4

SUBSTREAM MIXED TEMP $=25.0<\mathrm{C}>$ PRES $=1.0$ NPHASE $=1$ PHASE $=\mathrm{L}$ MASS-FLOW H2O $37.1 /$ HNO3 85.97

STREAM 12

SUBSTREAM MIXED TEMP=25.0 <C> PRES=1.0 MASS-FLOW=125\& SOLVENT $=$ H2O NPHASE $=1$ PHASE $=\mathrm{L}$

MOLE-CONC HNO3 $0.0500<$ MOL/L>

STREAM 17

SUBSTREAM MIXED TEMP $=25.0<\mathrm{C}>\mathrm{PRES}=1.0$ NPHASE $=1$ PHASE $=\mathrm{L}$

MASS-FLOW H2O $4.32 / \mathrm{NAOH} 1.740$

BLOCK TK-G-I MIXER

PARAM PRES $=1.0$

BLOCK TK-S-1 MIXER

PARAM PRES $=1.0$ NPHASE $=1$ PHASE $=\mathrm{L}$

BLOCK C-S-1 SEP

DESCRIPTION "SLUDGE WASH"

FRAC STREAM $=2$ SUBSTREAM $=$ MIXED COMPS $=\mathrm{H} 20 \mathrm{~F}-\mathrm{CL}-\mathrm{NA}+\mathrm{H} 3 \mathrm{O}+$ \& 
$\mathrm{OH}-\mathrm{NO} 3-\mathrm{CS}+\mathrm{CA}+2 \mathrm{SR}+2 \mathrm{MN}+2 \mathrm{FE}+3 \mathrm{CR}+3 \quad \mathrm{ZR}+4 \mathrm{MOO} 4-2$ \& $\mathrm{AL}+3 \mathrm{CO} 3-2$ PO4-3 SO4-2 NO2- "CSOH(S)" "PU(OH) 4" \& "U(OH) 4" "TH $(\mathrm{OH}) 4 "$ TCO4- FRACS $=0.83950 .40141 .000 .99 \&$ $0.0 \quad 0.5644 \quad 1.00 \quad 0.900 \quad 0.000 \quad 0.000 \quad 0.000 \quad 0.000 \quad 0.000 \quad \&$ $0.000 \quad 0.000 \quad 0.1378 \quad 0.2941 \quad 1.00 \quad 0.2866 \quad 1.00 \quad 0.900 \quad 0.00 \&$ $0.00 \quad 0.00 \quad 0.9999$

BLOCK F-A-1 SEP

FRAC STREAM $=20$ SUBSTREAM $=$ MIXED COMPS $=\mathrm{H} 2 \mathrm{O}$ NA+ $\mathrm{OH}-\mathrm{NO} 3-$ \& $\mathrm{TH}+4 \quad \mathrm{U}+4$ PU+4 "PU (OH) 4 " "U (OH) 4" $\mathrm{NP}+4 \quad \mathrm{AM}+3$ "TH(OH) $4 "$ \& "AM(OH) 3 " "NP $(\mathrm{OH}) 4$ " FRACS $=0.001 \quad 0.000 \quad 0.000 \quad 0.000 \quad 0.0 \&$ $0.00 .01 .001 .00 \quad 0.00 .01 .001 .001 .00$

BLOCK IX-A-1 SEP

FRAC STREAM $=14$ SUBSTREAM=MIXED COMPS $=\mathrm{H} 20 \mathrm{~F}-\mathrm{CL}-\mathrm{NA}+$ \& $\mathrm{H} 3 \mathrm{O}+\mathrm{NO} 3-\mathrm{CS}+\mathrm{CA}+2 \mathrm{SR}+2 \mathrm{MN}+2 \quad \mathrm{FE}+3 \quad \mathrm{CR}+3 \quad \mathrm{ZR}+4 \mathrm{MOO} 4-2$ \& $\mathrm{TH}+4 \quad \mathrm{U}+4 \quad \mathrm{AL}+3 \quad \mathrm{CO} 3-2 \quad \mathrm{PO} 4-3 \quad \mathrm{SO}_{1}-2$ NO2- $\mathrm{PU}+4 \quad \mathrm{NP}+4 \mathrm{AM}+3$ \& FRACS $=0.621 \quad 1.00 \quad 1.00 \quad 1.00 \quad 0.6449 \quad 0.99544 \quad 1.00 \quad 1.00 \quad \&$ $1.001 .00 \quad 1.00 \quad 1.001 .00 \quad 1.00 \quad 0.0 \quad 0.0 \quad 1.001 .00 \&$ $1.001 .001 .00 \quad 0.0 \quad 0.0 \quad 0.001$

BLOCK IX-C-1 SEP

FRAC STREAM $=24$ SUBSTREAM=MIXED COMPS $=\mathrm{H} 20$ HNO3 NAOH F- \& CL- NA+ H3O+ HF HCL "NACL(S)" "NANO3(S)" "NAF(S)" OH- \& NO3- CS+ CA+2 SR+2 MN+2 FE+3 CR+3 $\mathrm{ZR}+4$ MOO4-2 $\mathrm{TH}+4$ \& $\mathrm{U}+4$ AL+3 $\mathrm{CO} 3-2$ PO4-3 $\mathrm{SO} 4-2$ NO2- HNO2 "NANO2(S)" \& "CSOH(S) " $\mathrm{FU}+4$ "PU(OH) 4 " "U(OH) 4 " NP+4 AM+3 "TH(OH) 4 " \& "AM(OH) 3" "NP(OH) 4 " TCO4- FRACS $=0.00001 \quad 0.0 \quad 0.0 \quad 0.0 \&$ $\begin{array}{llllllllllllll}0.0 & 0.0 & 0.0 & 0.0 & 0.0 & 0.0 & 0.0 & 0.0 & 0.0 & 0.0 & 0.9999 & 0.0\end{array} \&$ $\begin{array}{lllllllllllll}0.0 & 0.0 & 0.0 & 0.0 & 0.0 & 0.0 & 0.0 & 0.0 & 0.0 & 0.0 & 0.0 & 0.0 & \&\end{array}$ $\begin{array}{lllllllllllll}0.0 & 0.0 & 0.0 & 0.9999 & 0.0 & 0.0 & 0.0 & 0.0 & 0.0 & 0.0 & 0.0 & 0.0 & \&\end{array}$ 0.0

BLOCK IX-S-1 SEP

FRAC STREAM=15 SUBSTREAM=MIXED COMPS=H20 HNO3 NAOH F- \& CL- NA+ H3O+ HF HCL "NACL(S)" "NANO3(S)" "NAF(S)" OH- \& NO3- CS+ CA+2 SR+2 MN+2 FE+3 CR+3 ZR+4 MOO4-2 TH+4 \& $\mathrm{U}+4 \mathrm{AL}+3 \quad \mathrm{CO} 3-2$ PO4-3 $\mathrm{SO} 4-2$ NO2- $\mathrm{HNO} 2$ "NANO2(S)" \& "CSOH(S)" $\mathrm{FU}+4$ "PU(OH) 4 " "U(OH) 4 " NP+4 AM+3 "TH(OH) 4 " \& "AM(OH) 3" "NP(OH) 4" TCO4- FRACS $=0.00010 .00 .00 .0$ \& $\begin{array}{llllllllllllllll}0.0 & 0.0 & 0.0 & 0.0 & 0.0 & 0.0 & 0.0 & 0.0 & 0.0 & 0.0 & 0.0 & 0.0\end{array}$ $\begin{array}{llllllllllllll}0.9999 & 0.0 & 0.0 & 0.0 & 0.0 & 0.0 & 0.0 & 0.0 & 0.0 & 0.0 & 0.0 & 0.0\end{array} \&$ $0.0 \quad 0.0 \quad 0.0 \quad 0.0 \quad 0.0 \quad 0.0 \quad 0.0 \quad 0.0 \quad 0.0 \quad 0.0 \quad 0.0 \quad 0.0 \quad \&$ 0.0

BLOCK IX-T-I SEP

FRAC STREAM $=26$ SUBSTREAM $=$ MIXED COMPS $=\mathrm{H} 20$ HNO3 NAOH F- \& CL- NAt H3O+ HF HCL "NACL(S) " "NANO3(S)" "NAF(S)" OH- \& NO3- CS+ $\mathrm{CA}_{2}+2 \mathrm{SR}+2 \mathrm{MN}+2 \mathrm{FE}+3 \mathrm{CR}+3 \quad \mathrm{ZR}+4 \mathrm{MOO} 4-2 \mathrm{TH}+4$ \& $\mathrm{U}+4$ AL+3 $\mathrm{CO}_{3-2} \mathrm{PO} 4-3$ SO4-2 NO2- HNO2 "NANO2(S)" \& "CSOH(S) " PU+4 "PU(OH) 4" "U(OH) 4" NP+4 AM+3 "TH(OH) 4 " \& "AM(OH) 3" "NP (OH) 4" TCO4- FRACS $=0.00001 \quad 0.00 .00 .0 \quad \&$ $\begin{array}{llllllllllllll}0.0 & 0.0 & 0.0 & 0.0 & 0.0 & 0.0 & 0.0 & 0.0 & 0.0 & 0.0 & 0.0 & 0.0 & \&\end{array}$

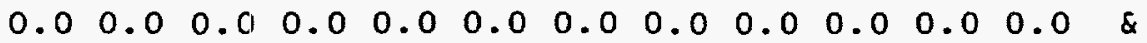
$\begin{array}{llllllllllllll}0.0 & 0.0 & 0.0 & 0.0 & 0.0 & 0.0 & 0.0 & 0.0 & 0.0 & 0.0 & 0.0 & 0.0 & \&\end{array}$ 0.9999

\section{BLOCK X-S-1 HEATER}

DESCRIPTION "STREAM COOLER"

PARAM TEMP $=30.0<\mathrm{C}>\mathrm{BRES}=1.0$ 
BLOCK SPLIT FLASH2

PARAM TEMP $=650.0<C>$ PRES $=1.0$

BLOCK RC-A-I RSTOIC

PARAM TEMP $=650.0<C>$ PRES $=1.0$

STOIC 1 MIXED "U(OH) 4" $-1 / \mathrm{H} 2 \mathrm{O} 2 / \mathrm{UO} 21$

STOIC 2 MIXED "TH(OH) 4" $-1 / \mathrm{H}_{2} \mathrm{O} 2 / \mathrm{THO} 21$

STOIC 3 MIXED "PU(OH) 4" -1/ $\mathrm{H} 20$ 2/ $/$ PUO2 1

STOIC 4 MIXED "AM(OH) 3" -2 / H2O 3/AM2O3 1

STOIC 5 MIXED "NP(OH) 4" -1/ $\mathrm{H} 2 \mathrm{O} 2 / \mathrm{NPO} 21$

CONV 1 MIXED "U(OH) $4 " 1.00$

CONV 2 MIXED "TH(OH) $4 " 1.00$

CONV 3 MIXED "PU(OH) 4 " 1.00

CONV 4 MIXED "AM(OH) $3 " 1.00$

CONV 5 MIXED "NP(OH) 4" 1.00

BLOCK TK-P-I RSTOIC

DESCRIPTION "PRECIPITATION"

PARAM TEMP $=25.0<C>$ PRES $=1.0$

STOIC 1 MIXED U+4 -1/OH- $-4 / " \mathrm{U}(\mathrm{OH}) 4 " 1$

STOIC 2 MIXED TH+4 -1/ OH- $4 /$ "TH(OH) $4 " 1$

STOIC 3 MIXED PU+4 -1/OH- $4 /$ "PU (OH) $4 " 1$

STOIC 4 MIXED NP+4 - / $\mathrm{OH}-4 / \mathrm{NP}(\mathrm{OH}) 4 " 1$

STOIC 5 MIXED AM+3-1/OH- $-3 /$ "AM(OH) 3" 1

CONV 1 MIXED U+4 0.999

CONV 2 MIXED TH+4 0.999

CONV 3 MIXED PU+4 0.999

CONV 4 MIXED NP+4 0.999

CONV 5 MIXED AM+3 0.999

STREAM-REPOR NOMOLEFLOW MASSFLOW NOMOLEFRAC INCL-STREAMS $=1 \quad 2 \quad 3$ \& $\begin{array}{lllllllllllllllll}4 & 5 & 11 & 12 & 13 & 14 & 15 & 16 & 17 & 18 & 19 & 20 & 21 & 22 & 24 & 25 & \text { \& }\end{array}$

$26 \quad 27 \quad 29$

PROPERTY-REP NOPARAMS PCES

;

;

;

; 


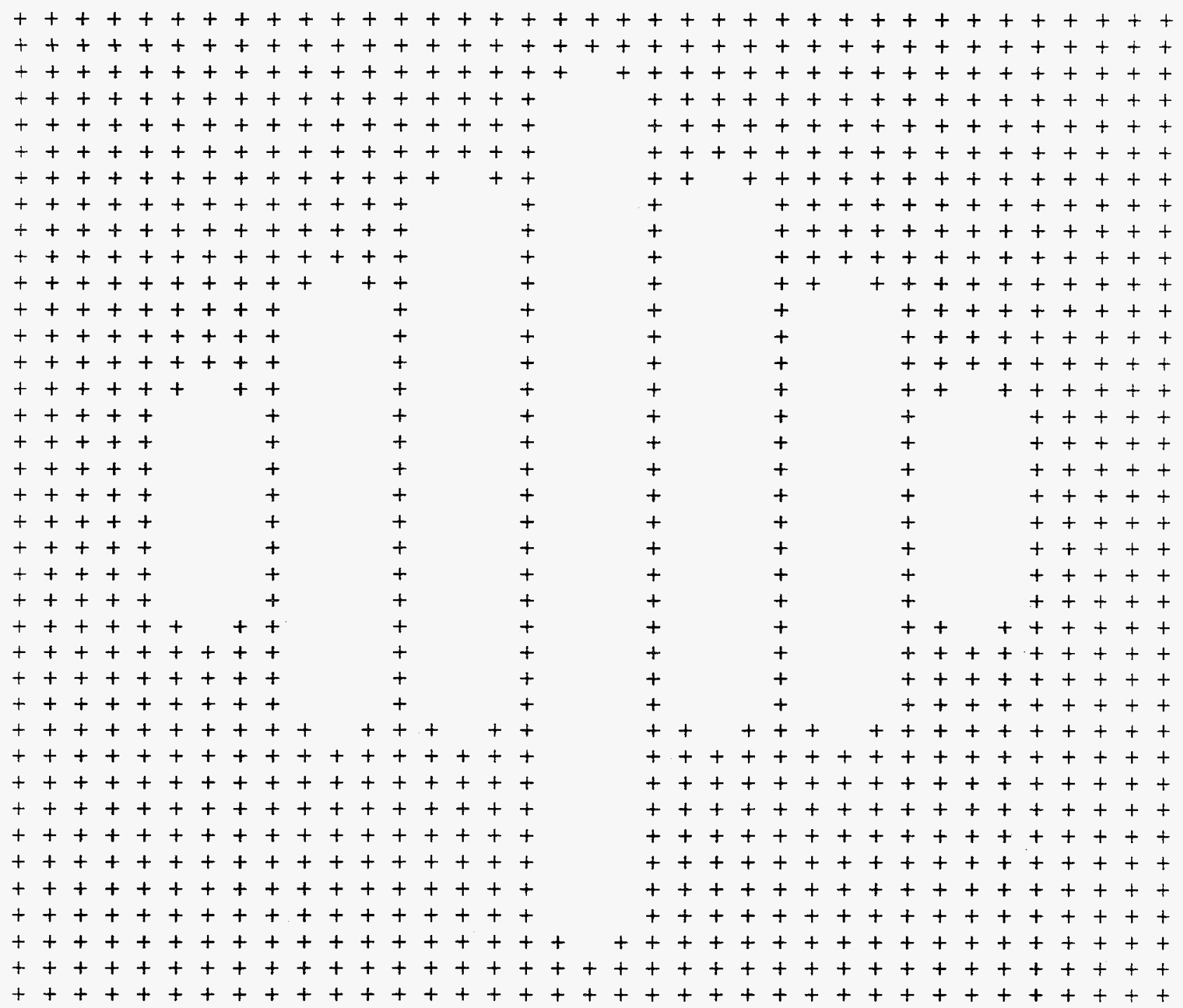

\begin{tabular}{|c|c|c|c|c|c|c|c|c|c|c|}
\hline AAAAA & SSSSS & PF'PPP & EEEEE & NN & $\mathrm{N}$ & PPPPP & $L$ & $\mathrm{U}$ & $\mathrm{U}$ & SSSSS \\
\hline A & $S$ & & $\mathrm{E}$ & $N \mathrm{~N}$ & $\mathrm{~N}$ & & $\mathrm{~L}$ & $\mathrm{U}$ & $\mathrm{U}$ & \\
\hline AAAAA & sSsss & PF'PPP & EEEEE & $\mathrm{N}$ & $\mathrm{N} \mathrm{N}$ & PPPPP & $\mathrm{L}$ & $\mathrm{U}$ & $\mathrm{U}$ & SSSSS \\
\hline A & $s$ & $\mathrm{P}$ & $E$ & $\mathrm{~N}$ & NN & $\mathbf{P}$ & $\mathrm{L}$ & $\mathrm{U}$ & $\mathrm{U}$ & S \\
\hline A & SSSSS & $P$ & EEEEE & $\mathrm{N}$ & $\mathrm{N}$ & $\mathbf{P}$ & LLLLI & UUT & UU & SSSSS \\
\hline
\end{tabular}

ASPEN PLUS IS A TFADEMARK OF ASPEN TECHNOLOGY, INC.

TEN CANAL PARK

CAMBRIDGE, MASSACHUSETTS 02141

617/577-0100

VERSION: DOS

RELEASE: $9.1-3$

INSTALLATION : LANI,-PC
HOTLINE :

U.S.A. $617 / 577-0337$

EUROPE (32) 2/726-9303

FEBRUARY 3， 1995

FRIDAY

10:55:01 A.M. 
ASPEN PLUS (TM) IS A PROPRIETARY PRODUCT OF ASPEN TECHNOLOGY, INC. (ASPENTECH), AND MAY BE USED ONLY UNDER AGREEMENT WITH ASPENTECH. RESTRICTED RIGHTS LEGEND: USE, REPRODUCTION, OR DISCLOSURE BY THE U.S. GOVERNMENT IS SUBJECT TO RESTRICTIONS SET FORTH IN

(i) FAR 52.227-14, Alt. III, (ii) FAR 52.227-19, (iii) DFARS

252.227-7013(c)(1)(ii), or (iv) THE ACCOMPANYING LICENSE AGREEMENT, AS APPLICABLE. FOR PURPOSES OF THE FAR, THIS SOFTWARE SHALL BE DEEMED TO BE "UNPUBLISHED" AND LICENSED WITH DISCLOSURE PROHIBITIONS. CONTRACTOR/SUBCONTRACTOR: ASPEN TECHNOLOGY, INC. TEN CANAL PARK, CAMBRIDGE, MA 02141.

\section{TABLE OF CONTENTS}

RUN CONTROL SECTION..................... I

RUN CONTROL INFORMATION..................

BLOCK STATUS....................... 2

FLOWSHEET SECTION.................... 3

FLOWSHEET CONNECTIVITY BY STREAMS........... 3

FLOWSHEET CONNECTIVITY BY BLOCKS............ 3

COMPUTATIONAL SEQUENCE................ 3

OVERALL FLOWSHEET BALANCE............... 3

PHYSICAL PROPERTIES SECTION................ 5

COMPONENTS........................... 5

PROPERTY CONSTANT ESTIMATION SECTION............ 6

PURE COMPONENT PARAMETERS................ 6

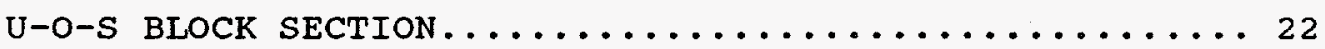

BLOCK: $C-S-1$ MODEL: SEP.............. 22

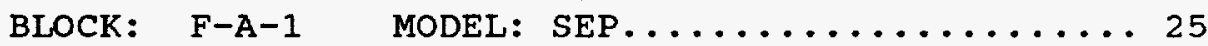

BLOCK: IX-A-1 MODEL: SEP.............. 28

BLOCK: IX-C-1 MODEL: SEP.............. 31

BLOCK: IX-S-1 MODEL: SEP.............. 35

BLOCK: IX-T-1 MODEL: SEP.............. 38

BLOCK: RC-A-1 MODEL: RSTOIC............ 42

BLOCK: SPLIT MODEL: FLASH $\ldots \ldots \ldots \ldots \ldots \ldots \ldots \ldots 44$

BLOCK: TK-G-1 MODEL: MIXER............. 45

BLOCK: TK-P-1 MODEL: RSTOIC........... 45

BLOCK: TK-S-1 MODEL: MIXER............. 48

BLOCK: $X-S-1$ MODEL: HEATER.............. 48

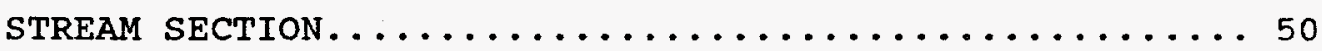

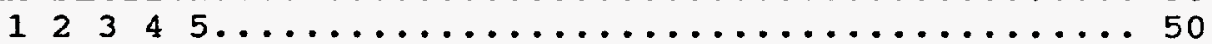

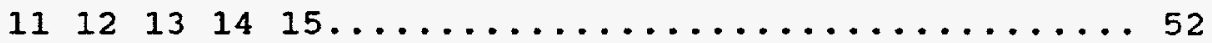

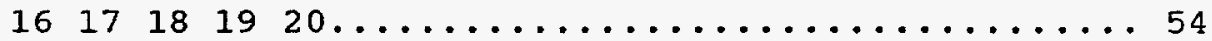

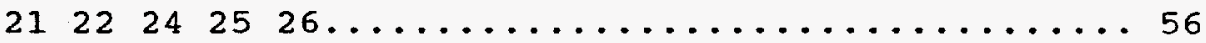

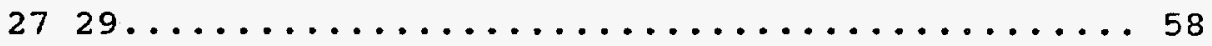


ASPEN PLUS VER: DOS

REL: $9.1-3$ INST: LANL-PC

TANK SY-102 CLEANOUT FLOWSHEET

RUN CONTROL SECTION

RUN CONTROL INFORNLATION

THIS COPY OF ASPEN PLUS LICENSED TO LOS ALAMOS NATIONAL LABORATORY

TYPE OF RUN: EDIT

INPUT FILE NAME:_unnamed.inm

INPUT PROBLEM DATA FILE NAME : _unnamed VERSION NO. 1 OUTPUT PROBLEM DAT'A FILE NAME: _unnamed VERSION NO. 1 LOCATED IN:

PDF SIZE USED FOR INPUT TRANSLATION: NUMBER OF FILE FECORDS (PSIZE) $=0$ NUMBER OF IN-COFE RECORDS $=256$ PSIZE NEEDED FOR SIMULATION = 1

CALLING PROGRAM NAME: apmain LOCATED IN: $\quad$ C: $\backslash A S P E N \backslash A P 91 / \mathrm{xeq}$

SIMULATION REQUESTED FOR ENTIRE FLOWSHEET 
ASPEN PLUS VER: DOS
REL: 9.1-3 INST: LANL-PC
TANK SY-102 CLEANOUT FLOWSHEET
RUN CONTROL SECTION

\section{BLOCK STATUS}

--ー-ー------

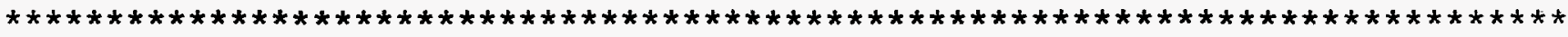

\section{$\star$}

* ALL UNIT OPERATION BLOCKS WERE COMPLETED NORMALLY

*

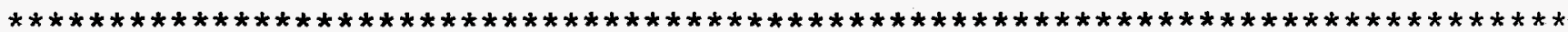


ASPEN PLUS VER: DOS

REL: $9 \cdot 1-3$

INST: LANL-PC

$02 / 03 / 95$

PAGE 3

TANK SY-102 CLEANOUT FLOWSHEET

FLOWSHEET SECTION

FLOWSHEET CONNECTIVITY BY STREAMS

$\begin{array}{llllll}\text { STREAM } & \text { SOURCE } & \text { DEST } & \text { STREAM } & \text { SOURCE } & \text { DEST } \\ 1 & --- & \text { C-S-1 } & 4 & --- & \text { TK-S-1 } \\ 12 & \text { IX-A-1 } & 17 & --- & \text { TK-P-1 } \\ 2 & \text { C-S-1 } & \text { IX-C-1 } & 3 & \text { C-S-1 } & \text { TK-S-1 } \\ 5 & \text { TK-S-1 } & X-S-1 & 13 & \text { IX-A-1 } & \text { TK-P-1 } \\ 14 & \text { IX-A-1 } & \text { IX-S-1 } & 15 & \text { IX-S-1 } & ----- \\ 16 & \text { IX-S-1 } & \text { TK-G-1 } & 25 & \text { IX-C-1 } & \text { IX-T-1 } \\ 24 & \text { IX-C-1 } & --- & 26 & \text { IX-T-1 } & --- \\ 27 & \text { IX-T-1 } & \text { TK-G-1 } & 18 & \text { TK-P-1 } & \text { F-A-1 } \\ 19 & \text { F-A-1 } & \text { TK-G-1 } & 20 & \text { F-A-1 } & \text { RC-A-1 } \\ 29 & \text { TK-G-1 } & ---- & 11 & \text { X-S-1 } & \text { IX-A-1 } \\ 21 & \text { SPLIT } & --- & 22 & \text { SPLIT } & ---- \\ 30 & \text { RC-A-1 } & \text { SPLIT } & & & \end{array}$

FLOWSHEET CONNECTIVITY BY BLOCKS

\begin{tabular}{|c|c|c|}
\hline BLOCK & INLETS & OUTLETS \\
\hline$c-S-1$ & 1 & 23 \\
\hline TK-S-1 & 43 & 5 \\
\hline$I X-A-1$ & $11 \quad 12$ & $13 \quad 14$ \\
\hline$I X-S-1$ & 14 & $15 \quad 16$ \\
\hline$I X-C-1$ & 2 & $25 \quad 24$ \\
\hline $\mathrm{IX}-\mathrm{T}-\mathrm{I}$ & 25 & $26 \quad 27$ \\
\hline $\mathrm{TK}-\mathrm{P}-\mathrm{I}$ & $13 \quad 17$ & 18 \\
\hline$F-A-1$ & 18 & 1920 \\
\hline$T K-G-1$ & $\begin{array}{lll}19 & 27 & 16\end{array}$ & 29 \\
\hline$R C-A-1$ & 20 & 30 \\
\hline$x-s-1$ & 5 & 11 \\
\hline SPLIT & 30 & $21 \quad 22$ \\
\hline
\end{tabular}

COMPUTATIONAL SEQUENCE

SEQUENCE USED WAS:

C-S-1 IX-C-1 IX-T-1 TK-S-1 X-S-1 IX-A-1 TK-P-1 F-A-1 RC-A-1 SPLIT IX $-\mathrm{S}-1$ TK $-\mathrm{G}-1$

OVERALL FLOWSHEET BALANCE

*** MASS AND ENERGY BALANCE IN OUT

CONVENTIONAL COMPONENTS (KMOL/HR)

H2O
HNO3
NAOH
F-
CL-
NA+
H3O+
HF

60.0566

$0.000000 \mathrm{E}+00$

$0.000000 \mathrm{E}+00$

$0.747410 \mathrm{E}-01$

$0.375142 \mathrm{E}-01$

1.64422

$0.308221 \mathrm{E}-10$

$0.168101 \mathrm{E}-12$ $\star * *$

GENERATION RELATIVE DIFF.
$0.121351 \mathrm{E}-01$
$-0.411125 \mathrm{E}-01$
$0.000000 E+00$
1.00000
$0.000000 \mathrm{E}+00$
$0.000000 E+00$
$0.000000 \mathrm{E}+00$
$0.000000 E+00$
$0.325253 E-11$
$0.000000 \mathrm{E}+00$
$-0.924836 \mathrm{E}-15$
$0.000000 \mathrm{E}+00$
$0.000000 \mathrm{E}+00$
$0.000000 \mathrm{E}+00$
1. 00000
$-1.00000$ 
REL: 9.1-3

INST: LANL-PC

02/03/95 PAGE 4

TANK SY-102 CLEANOUT FLOWSHEET

FLOWSHEET SECTION

OVERALL FLOWSHEET BALANCE (CONTINUED)

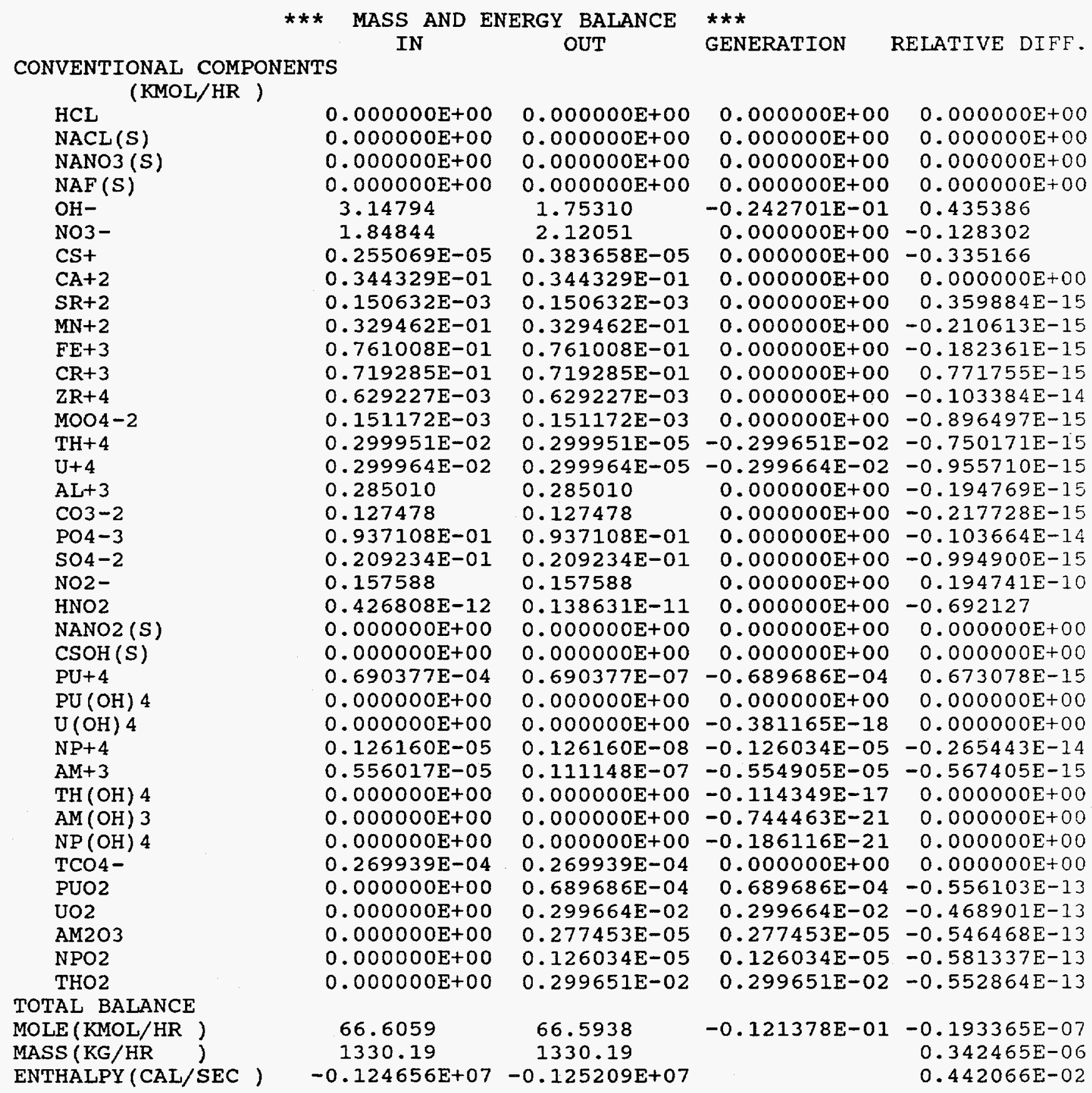


REL: $9.1-3$

INST : LANL-PC

$02 / 03 / 95$

PAGE 5

TANK SY-102 CLEANOUT FLOWSHEET

PHYSICAL PROPERTIES SECTION

COMPONENTS

\begin{tabular}{|c|c|c|c|c|}
\hline ID & TYPE & FOIRMULA & NAME OR ALIAS & REPORT NAME \\
\hline $\mathrm{H} 2 \mathrm{O}$ & C & $\mathrm{H} 2 \mathrm{O}$ & H2O & $\mathrm{H} 2 \mathrm{O}$ \\
\hline HNO3 & C & HeNO3 & HNO3 & HNO3 \\
\hline $\mathrm{NAOH}$ & C & $\mathrm{NAOH}$ & $\mathrm{NAOH}$ & $\mathrm{NAOH}$ \\
\hline F- & c & F- & F- & F- \\
\hline CL- & c & $C: L-$ & CL- & CL- \\
\hline NAt & c & NAt & $\mathrm{NA}+$ & $\mathrm{NA}+$ \\
\hline $\mathrm{H} 3 \mathrm{O}+$ & C & $\mathrm{H} 3 \mathrm{O}+$ & $\mathrm{H} 3 \mathrm{O}+$ & $\mathrm{H} 3 \mathrm{O}+$ \\
\hline HF & C & $\mathrm{HEF}$ & $\mathrm{HF}$ & $\mathrm{HF}$ \\
\hline HCL & C & HCL & HCL & HCL \\
\hline $\operatorname{NACL}(\mathrm{S})$ & c & NACL & NACL & NACL (S) \\
\hline NANO3 (S) & C & NANO3 & NANO3 & NANO3 (S) \\
\hline $\operatorname{NAF}(\mathrm{S})$ & C & NAF & NAF & $\operatorname{NAF}(S)$ \\
\hline $\mathrm{OH}-$ & C & OHA- & $\mathrm{OH}-$ & $\mathrm{OH}-$ \\
\hline NO3- & C & NO3- & NO3- & NO3- \\
\hline CSt & C & $c \vdots 3+$ & CS+ & cst \\
\hline $\mathrm{CA}+2$ & C & $\mathrm{CAA}+2$ & $C A+2$ & $\mathrm{CA}+2$ \\
\hline $\mathrm{SR}+2$ & C & $\mathrm{SlR}+2$ & $S R+2$ & $S R+2$ \\
\hline $\mathrm{MN}+2$ & c & $\mathrm{M}] \mathrm{N}+2$ & $\mathrm{MN}+2$ & $M N+2$ \\
\hline $\mathrm{FE}+3$ & C & $F] Q+3$ & $\mathrm{FE}+3$ & $\mathrm{FE}+3$ \\
\hline$C R+3$ & c & $\mathrm{ClR}+3$ & $\mathrm{CR}+3$ & $C R+3$ \\
\hline $\mathrm{ZR}+4$ & c & $2 R+4$ & $\mathrm{ZR}+4$ & $\mathrm{ZR}+4$ \\
\hline MOO4-2 & C & $\mathrm{MOO4-2}$ & MOO4-2 & MOO4-2 \\
\hline $\mathrm{TH}+4$ & C & $\mathrm{TH}+4$ & $\mathrm{TH}+4$ & $\mathrm{TH}+4$ \\
\hline $\mathrm{U}+4$ & c & $U+4$ & $\mathrm{U}+4$ & $\mathrm{U}+4$ \\
\hline$A L+3$ & C & $A: L+3$ & $\mathrm{AL}+3$ & $A L+3$ \\
\hline $\mathrm{CO} 3-2$ & c & $\cos -2$ & $\mathrm{CO} 3-2$ & $\mathrm{CO} 3-2$ \\
\hline PO4-3 & C & PO4-3 & PO4-3 & $\mathrm{PO} 4-3$ \\
\hline SO4-2 & C & SO4-2 & SO4-2 & $\mathrm{SO}_{4-2}$ \\
\hline NO2- & c & NO2- & NO2- & NO2- \\
\hline HNO2 & C & $\mathrm{HNO} 2$ & HNO2 & HNO2 \\
\hline NANO2 (S) & c & NANO2 & NANO2 & NANO2 (S) \\
\hline $\mathrm{CSOH}(\mathrm{S})$ & C & $\mathrm{C}: \mathrm{SOH}$ & $\mathrm{CSOH}$ & $\mathrm{CSOH}(\mathrm{S})$ \\
\hline $\mathrm{PU}+4$ & c & MISSING & MISSING & $\mathrm{PU}+4$ \\
\hline $\mathrm{PU}(\mathrm{OH}) 4$ & C & MISSING & MISSING & $\mathrm{PU}(\mathrm{OH}) 4$ \\
\hline $\mathrm{U}(\mathrm{OH}) 4$ & c & $\mathrm{U}(\mathrm{OH}) 4$ & $\mathrm{U}(\mathrm{OH}) 4$ & $\mathrm{U}(\mathrm{OH}) 4$ \\
\hline $\mathrm{NP}+4$ & C & MISSING & MISSING & $\mathrm{NP}+4$ \\
\hline $\mathrm{AM}+3$ & C & MISSING & MISSING & $\mathrm{AM}+3$ \\
\hline $\mathrm{TH}(\mathrm{OH}) 4$ & C & MISSING & MISSING & $\mathrm{TH}(\mathrm{OH}) 4$ \\
\hline$A M(\mathrm{OH}) 3$ & C & MISSING & MISSING & $\mathrm{AM}(\mathrm{OH}) 3$ \\
\hline $\mathrm{NP}(\mathrm{OH}) 4$ & C & MISSING & MISSING & $\mathrm{NP}(\mathrm{OH}) 4$ \\
\hline TCO4- & c & MISSING & MISSING & $\mathrm{TCO}_{4-}$ \\
\hline PUO2 & $c$ & PIJO2 & PUO2 & PUO2 \\
\hline UO2 & C & UO2 & UO2 & vo2 \\
\hline AM203 & C & MISSING & MISSING & AM2O3 \\
\hline NPO2 & C & N:PO2 & NPO2 & NPO2 \\
\hline THO2 & C & THO2 & THO2 & THO2 \\
\hline $\begin{array}{l}\text { LISTID } \\
\text { GLOBAL }\end{array}$ & & $\begin{array}{c}\text { SUPERCR } \\
\text { HCL }\end{array}$ & OMPONENT LIST & \\
\hline
\end{tabular}


ASPEN PLUS VER: DOS

REL: $9.1-3$

INST: LANL-PC

$02 / 03 / 95$

PAGE 6

TANK SY-102 CLEANOUT FLOWSHEET

PROPERTY CONSTANT ESTIMATION SECTION

PURE COMPONENT PARAMETERS

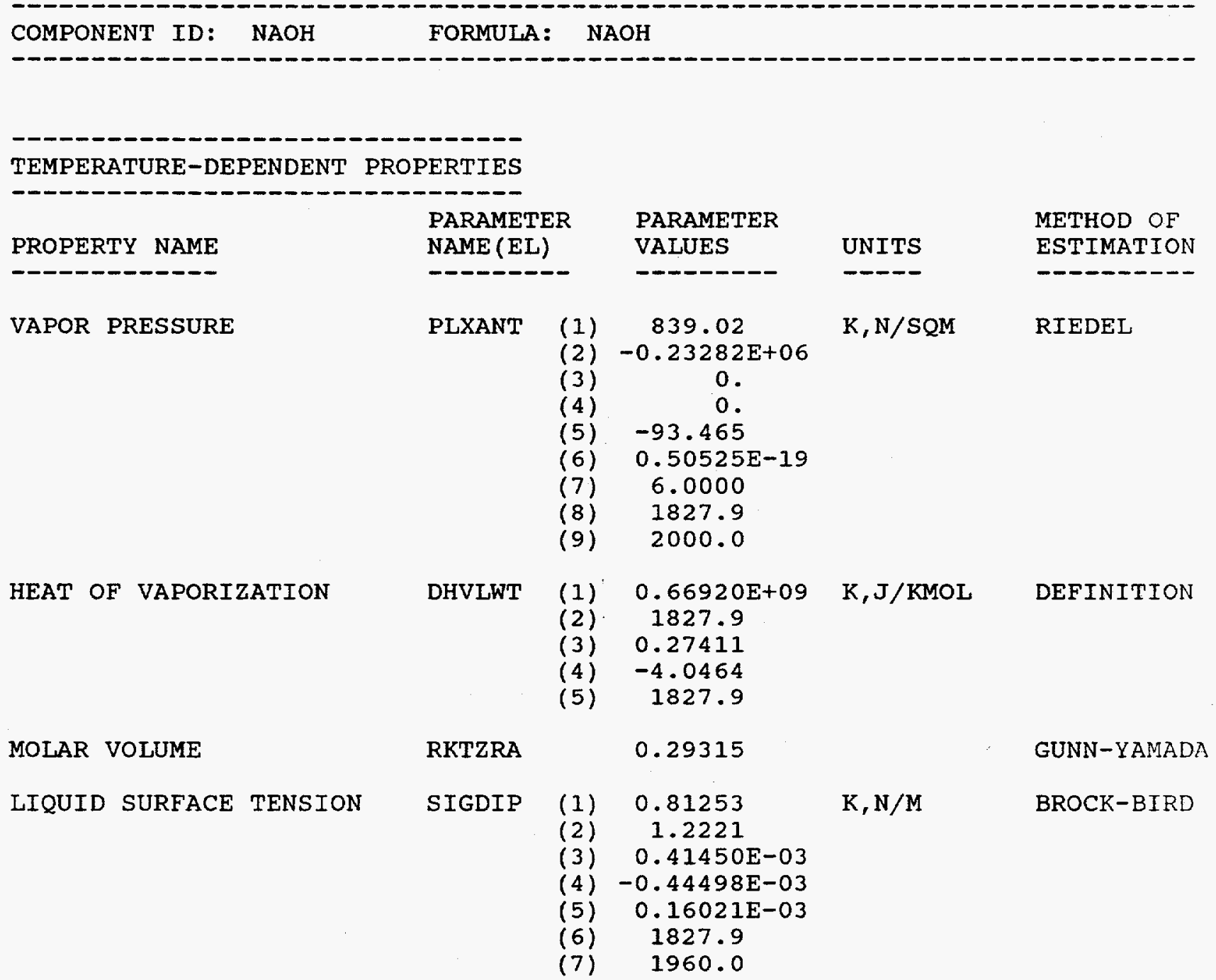


ASPEN PLUS VER: DOS

REL: 9.1-3 INST: LANL-PC

$02 / 03 / 95$

PAGE 7

TANK SY-102 CLEANOUT FLOWSHEET

PROPERTY CONSTANT ESTIMATION SECTION

PURE COMPONENT PARAMETERS (CONTINUED)

DEVIATIONS IN REGRESSING TEMPERATURE-DEPENDENT PROPERTIES

HEAT OF VAPORIZATION

METHOD $=$ DEFINITI

POINT TEMP

No. (K)

$1 \quad 1827.91$

1844.81

1861.71

1878.61

1895.51

1912.40

1929.30

1946.20

1963.10

1980.00

HEAT OF VAPORIZATION ( J / KMOL

DATA CALCULATED

\% ERROR

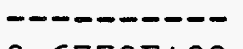

$0.6779 \mathrm{E}+09$

CALCULATED

$0.6719 \mathrm{E}+09$

$0.6692 \mathrm{E}+09$

$0.6646 \mathrm{E}+09$

$0.6720 \mathrm{E}+09$

$0.6551 E+09$

$0.6700 \mathrm{E}+09$

$0.6424 \mathrm{E}+09$

$0.6626 \mathrm{E}+09$

- - - -

$-1.29$

0.01

0.82

1.13

0.96

$0.6245 \mathrm{E}+09$

$0.6486 \mathrm{E}+09$

0.37

$0.5986 \mathrm{E}+09$

$0.6268 \mathrm{E}+09$

$0.5600 \mathrm{E}+09$

.5955E+09

$-0.51$

$0.4999 \mathrm{E}+09$

$0.5522 \mathrm{E}+09$

$-1.39$

$0.3988 \mathrm{E}+09$

$0.4922 \mathrm{E}+09$

$-1.53$

$0.4047 \mathrm{E}+09$

1.48

MOLAR VOLUME

METHOD = GUNN-YAM

$\begin{array}{cc}\text { POINT } & \text { TEMP } \\ \text { NO. } & (\mathrm{K}) \\ ----- & --.91 \\ 1 & 1827.91 \\ 2 & 1844.81 \\ 3 & 1861.71 \\ 4 & 1878.61 \\ 5 & 1895.51 \\ 6 & 1912.40 \\ 7 & 1929.30 \\ 8 & 1946.20 \\ 9 & 1963.10 \\ 10 & 1980.00\end{array}$

MOLAR VOLUME

(CUM/KMOL

\begin{tabular}{lll} 
DATA & CALCULATED & $\%$ ERROR \\
\hdashline 0.5275 & --5.5303 & 0.53 \\
0.5370 & 0.5398 & 0.52 \\
0.5475 & 0.5503 & 0.50 \\
0.5594 & 0.5619 & 0.45 \\
0.5729 & 0.5750 & 0.36 \\
0.5888 & 0.5901 & 0.22 \\
0.6080 & 0.6080 & 0.00 \\
0.6321 & 0.6299 & -0.34 \\
0.6644 & 0.6587 & -0.86 \\
0.7136 & 0.7015 & -1.70
\end{tabular}


ASPEN PLUS VER: DOS

REL: $9 \cdot 1-3$

INST: LANL-PC

TANK SY-102 CLEANOUT FLOWSHEET

PROPERTY CONSTANT ESTIMATION SECTION

PURE COMPONENT PARAMETERS (CONTINUED)

LIQUID SURFACE TENSION

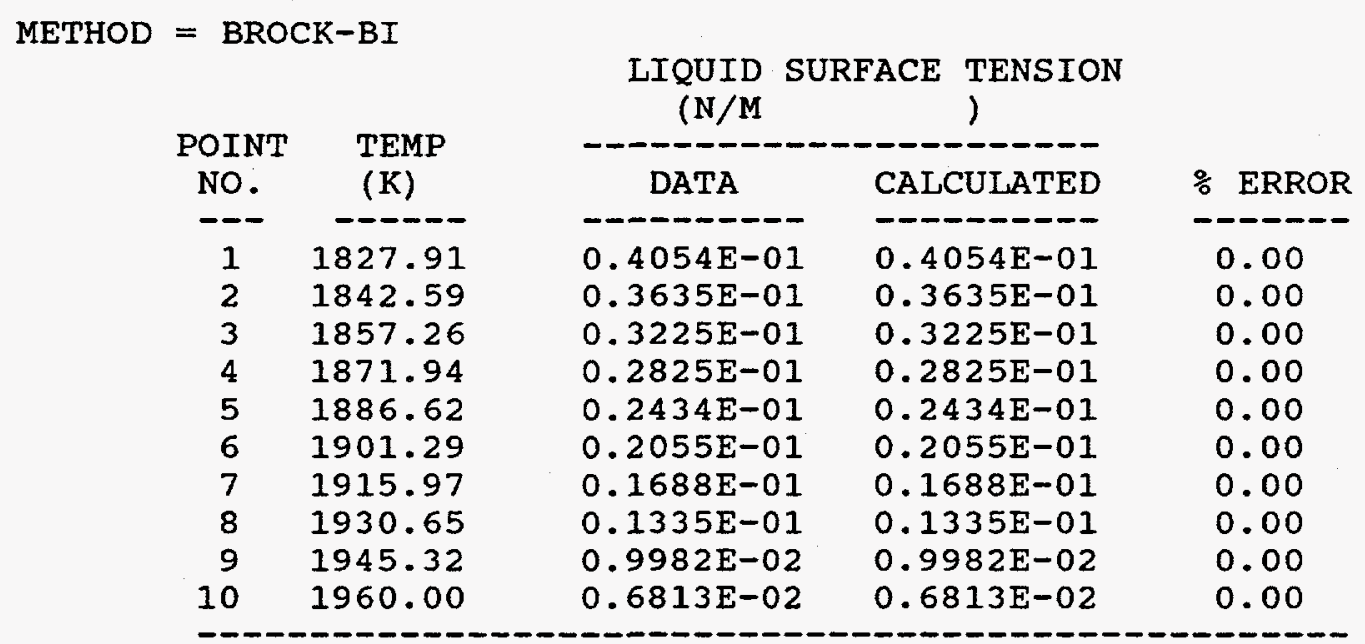


ASPEN PLUS VER: DOS

REL: $9.1-3$

INST: LANL-PC

$02 / 03 / 95$

PAGE 9

TANK SY-102 CLEANOUT FLOWSHEET

PROPERTY CONSTANT ESTIMATION SECTION

PURE COMPONENT PARAMETERS (CONTINUED)

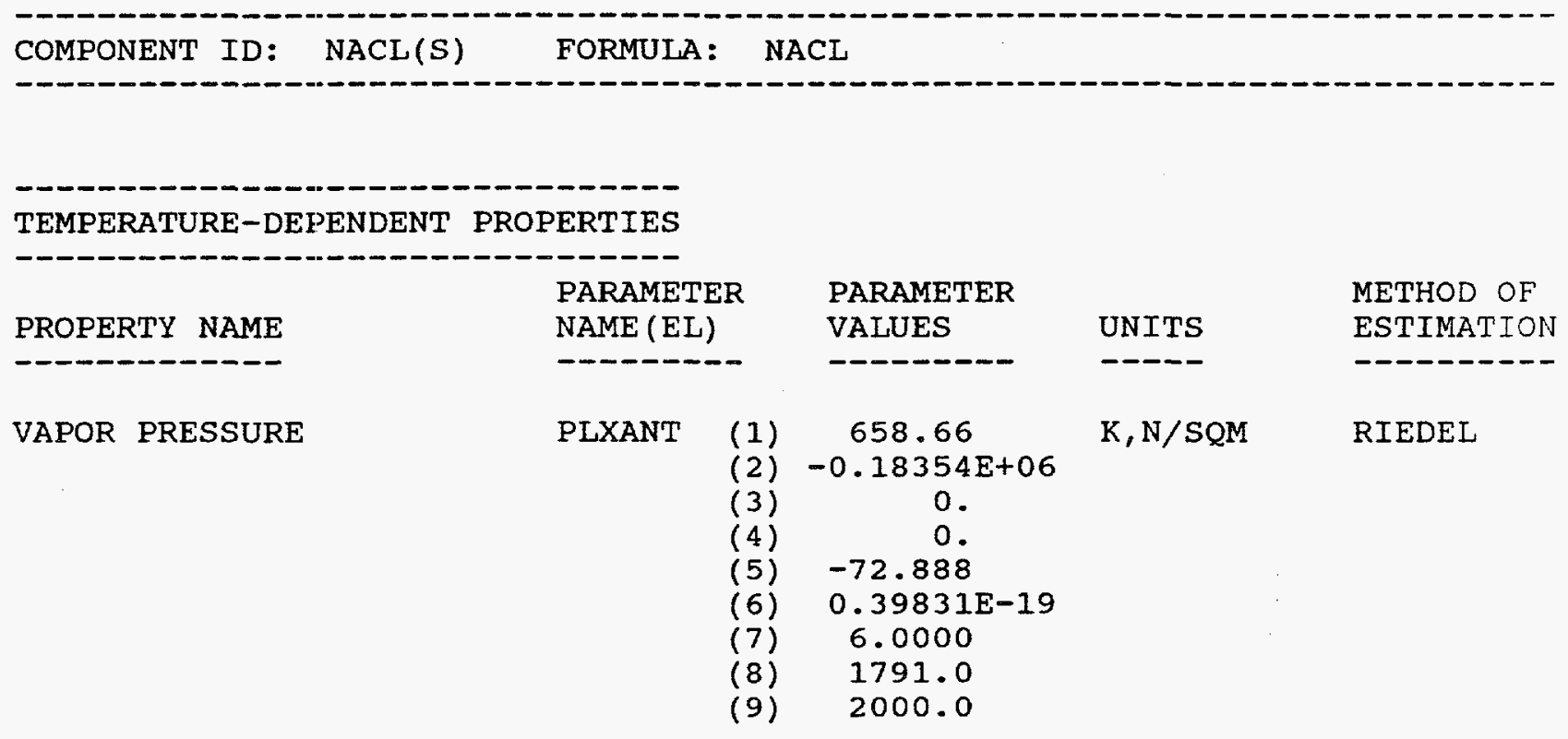


ASPEN PLUS VER: DOS

REL: 9.1-3 INST: LANL-PC

TANK SY-102 CLEANOUT FLOWSHEET

PROPERTY CONSTANT ESTIMATION SECTION

PURE COMPONENT PARAMETERS (CONTINUED)

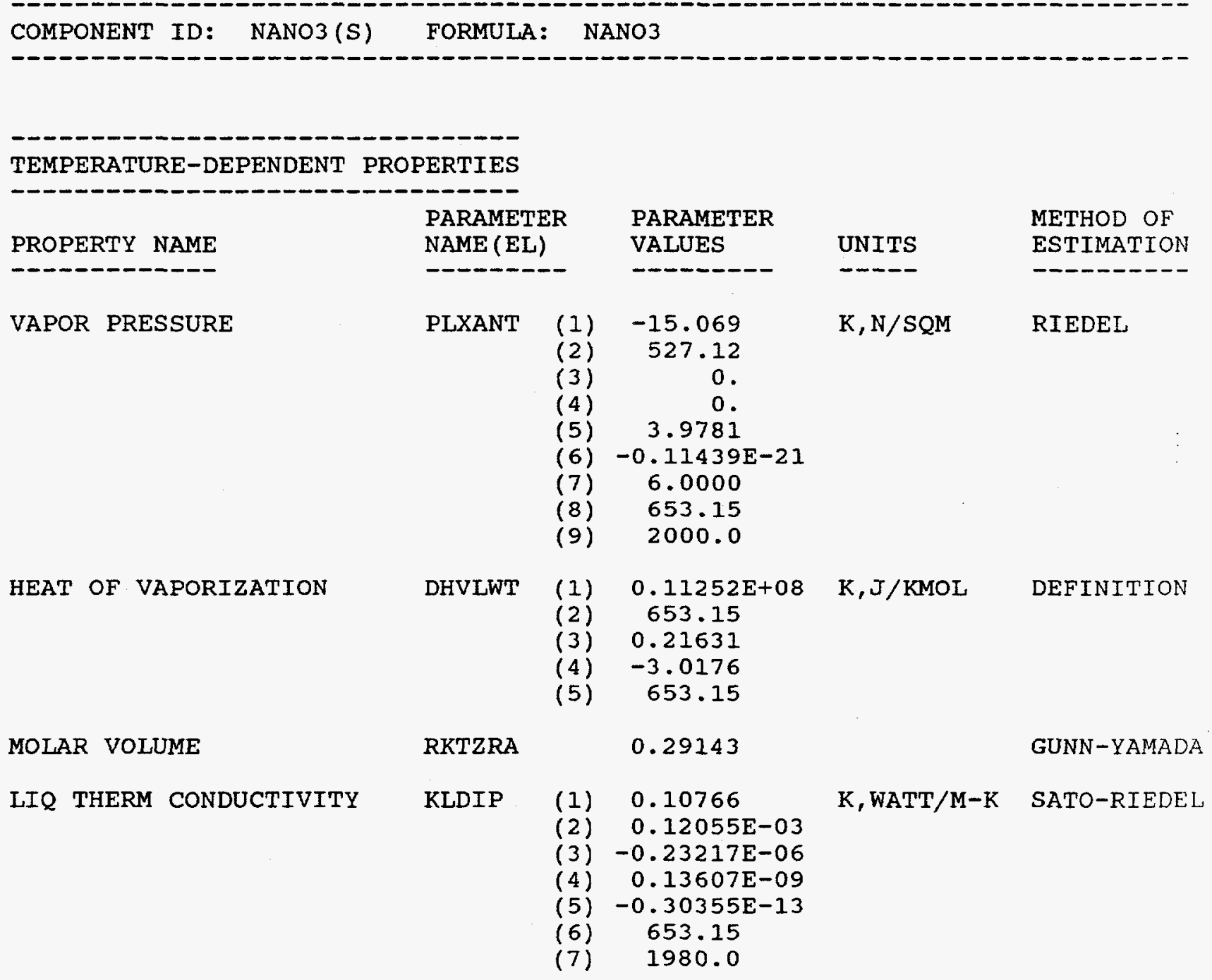


ASPEN PLUS VER: DOS

REL: $9.1-3$

INST: LANL-PC

$02 / 03 / 95$

PAGE 11

TANK SY-102 CLEANOUT FLOWSHEET

PROPERTY CONSTANT ESTIMATION SECTION

PURE COMPONENT PARAMETERS (CONTINUED)

DEVIATIONS IN REGRESSING TEMPERATURE-DEPENDENT PROPERTIES

HEAT OF VAPORIZATION

METHOD $=$ DEFINITI

HEAT OF VAPORIZATION

(J/KMOL

\begin{tabular}{ccccc} 
POINT & TEMP & - & - & \\
NO. & $(\mathrm{K})$ & DATA & CALCULATED & $\%$ \\
\hdashline & $-1 .-15 R O R$ \\
\hdashline. & 653.15 & $0.1115 \mathrm{E}+08$ & $0.1125 \mathrm{E}+08$ & 0.89 \\
2 & 800.58 & $0.1379 \mathrm{E}+08$ & $0.1354 \mathrm{E}+08$ & -1.84 \\
3 & 948.01 & $0.1595 \mathrm{E}+08$ & $0.1579 \mathrm{E}+08$ & -1.02 \\
4 & 1095.43 & $0.1759 \mathrm{E}+08$ & $0.1777 \mathrm{E}+08$ & 1.06 \\
5 & 1242.86 & $0.1863 \mathrm{E}+08$ & $0.1918 \mathrm{E}+08$ & 2.98 \\
6 & 1390.29 & $0.1896 \mathrm{E}+08$ & $0.1965 \mathrm{E}+08$ & 3.64 \\
7 & 1537.72 & $0.1843 \mathrm{E}+08$ & $0.1882 \mathrm{E}+08$ & 2.12 \\
8 & 1685.14 & $0.1676 \mathrm{E}+08$ & $0.1639 \mathrm{E}+08$ & -2.19 \\
9 & 1832.57 & $0.1330 \mathrm{E}+08$ & $0.1214 \mathrm{E}+08$ & -8.77 \\
10 & 1980.00 & $0.4952 \mathrm{E}+07$ & $0.5140 \mathrm{E}+07$ & 3.78 \\
\hline
\end{tabular}

MOLAR VOLUME

\begin{tabular}{|c|c|c|c|c|}
\hline & & $\begin{array}{l}\text { MOLAR VOI } \\
\text { (CUM } / \mathrm{KI}\end{array}$ & LE & \\
\hline POINT & TEMP & ----- & 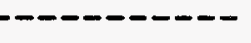 & \\
\hline No. & (K) & DATA & CALCULATED & \% ERROR \\
\hline$\cdots$ & ---- & $-\infty-----$ & $-----\infty---$ & ---- \\
\hline 1. & 653.15 & 0.3197 & 0.3222 & 0.78 \\
\hline 2 & 800.58 & 0.3323 & 0.3340 & 0.51 \\
\hline 3 & 948.01 & 0.3466 & 0.3473 & 0.20 \\
\hline 4. & 1095.43 & 0.3625 & 0.3627 & 0.06 \\
\hline 5 & 1242.86 & 0.3805 & 0.3808 & 0.07 \\
\hline 6 & 1390.29 & 0.4021 & 0.4028 & 0.17 \\
\hline 7 & 1537.72 & 0.4292 & 0.4306 & 0.32 \\
\hline$\varepsilon$ & 1685.14 & 0.4691 & 0.4685 & -0.14 \\
\hline 9 & 1832.57 & 0.5301 & 0.5282 & -0.35 \\
\hline 10 & 1980.00 & 0.7136 & 0.6963 & -2.43 \\
\hline
\end{tabular}


REL: $9.1-3$

TANK SY-102 CLEANOUT FLOWSHEET

PROPERTY CONSTANT ESTIMATION SECTION

PURE COMPONENT PARAMETERS (CONTINUED)

LIQ THERM CONDUCTIVITY

METHOD = SATO-RIE

\begin{tabular}{|c|c|c|c|c|}
\hline \multirow{2}{*}{$\begin{array}{c}\text { POINT } \\
\text { NO. }\end{array}$} & \multirow{2}{*}{$\begin{array}{l}\text { TEMP } \\
\text { (K) }\end{array}$} & \multicolumn{2}{|l|}{$-\ldots$} & \multirow[b]{2}{*}{$\%$ ERROR } \\
\hline & & DATA & CALCULATED & \\
\hline--- & & & & \\
\hline 1 & 653.15 & 0.1199 & 0.1198 & -0.12 \\
\hline 2 & 800.58 & 0.1124 & 0.1127 & 0.26 \\
\hline 3 & 948.01 & 0.1047 & 0.1047 & 0.05 \\
\hline 4 & 1095.43 & $0.9651 \mathrm{E}-01$ & $0.9628 E-01$ & -0.24 \\
\hline 5 & 1242.86 & $0.8791 \mathrm{E}-01$ & $0.8767 \mathrm{E}-01$ & -0.27 \\
\hline 6 & 1390.29 & $0.7872 \mathrm{E}-01$ & $0.7877 \mathrm{E}-01$ & 0.05 \\
\hline 7 & 1537.72 & $0.6876 \mathrm{E}-01$ & $0.6911 E-01$ & 0.51 \\
\hline 8 & 1685.14 & $0.5765 \mathrm{E}-01$ & $0.5789 E-01$ & 0.42 \\
\hline 9 & 1832.57 & $0.4457 \mathrm{E}-01$ & $0.4397 E-01$ & -1.34 \\
\hline 10 & 1980.00 & $0.2564 \mathrm{E}-01$ & $0.2587 E-01$ & 0.87 \\
\hline
\end{tabular}


ASPEN PLUS VER: DOS

REL: $9.1-3$

INST: LANL-PC

$02 / 03 / 95$

PAGE 13 TANK SY-102 CLEANOUT FLOWSHEET PROPERTY CONSTANT ESTIMATION SECTION

PURE COMPONENT PARAMETERS (CONTINUED)

COMPONENT ID: NAF (S)

FORMULA: NAF

TEMPERATURE-DEPENDENT PROPERTIES

TEMPEATURE

PROPERTY NAME

- - - - - - - -

VAPOR PRESSURE
PARAMETER

NAME (EL)

- - - - - -

PLXANT
PARAMETER

VALUES

-------

(1) 9823.8

(2) $-0.26875 E+07$

(3) 0 .

(4) 0 .

(5) -1118.5

(6) $0.58323 \mathrm{E}-18$

(7) 6.0000

(8) 1982.7

(9) 2000.0
METHOD OF ESTIMATION

UNITS

RIEDEL

$\mathrm{K}, \mathrm{N} / \mathrm{SQM}$ 
ASPEN PLUS VER: DOS

REL: $9 \cdot 1-3$

INST: LANL-PC

$02 / 03 / 95$

PAGE 14

TANK SY-102 CLEANOUT FLOWSHEET

PROPERTY CONSTANT ESTIMATION SECTION

PURE COMPONENT PARAMETERS (CONTINUED)

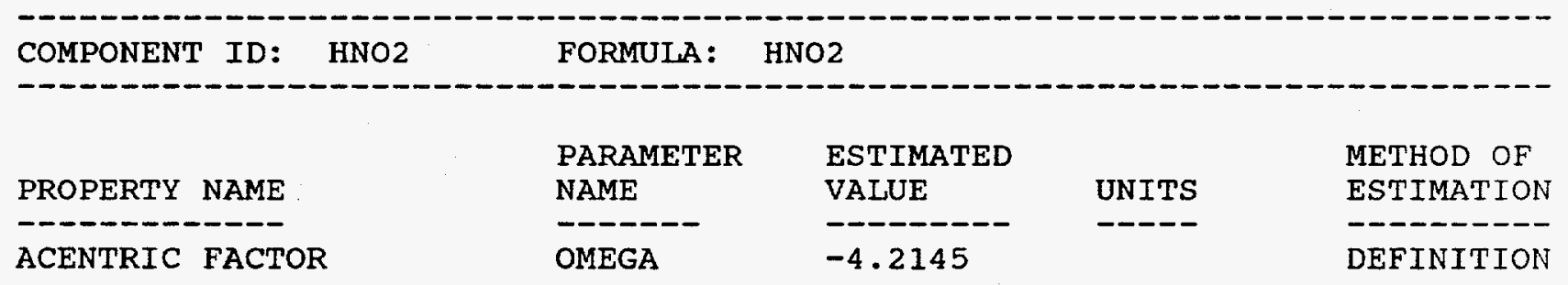




\section{PURE COMPONENT PARAMETERS (CONTINUED)}

TEMPERATURE-DEPENDENT PROPERTIES

\begin{tabular}{|c|c|c|c|c|c|}
\hline PROPERTY NAME & $\begin{array}{l}\text { PARAMET } \\
\text { NAME (EI }\end{array}$ & & $\begin{array}{l}\text { PARAMETER } \\
\text { VALUES }\end{array}$ & $\begin{array}{l}\text { UNITS } \\
----\end{array}$ & $\begin{array}{l}\text { METHOD OF } \\
\text { ESTIMATION } \\
-\end{array}$ \\
\hline LIQUID VISCOSITY & MULAND & $\begin{array}{l}(1) \\
(2) \\
(3) \\
(4) \\
(5)\end{array}$ & $\begin{array}{r}23.732 \\
-17125 . \\
0 \\
364.00 \\
514.80\end{array}$ & $\mathrm{~K}, \mathrm{~N}-\mathrm{SEC} / \mathrm{SQM}$ & LETSOU-STIE \\
\hline LIQUID SURFACE TENSION & SIGDIP & $\begin{array}{l}(1) \\
(2) \\
(3) \\
(4) \\
(5) \\
(6) \\
(7)\end{array}$ & $\begin{array}{c}0.10000 \mathrm{E}+36 \\
0.91762 \mathrm{E}-10 \\
-0.40960 \mathrm{E}-10 \\
-0.39147 \mathrm{E}-10 \\
0 . \\
364.00 \\
509.60\end{array}$ & $\mathrm{~K}, \mathrm{~N} / \mathrm{M}$ & HAKIM-ST \\
\hline
\end{tabular}




\section{ASPEN PLUS VER: DOS \\ REL: $9.1-3$ \\ INST: LANL-PC \\ $02 / 03 / 95$ \\ PAGE 16 \\ TANK SY-102 CLEANOUT FLOWSHEET \\ PROPERTY CONSTANT ESTIMATION SECTION \\ PURE COMPONENT PARAMETERS (CONTINUED)}

DEVIATIONS IN REGRESSING TEMPERATURE-DEPENDENT PROPERTIES

LIQUID VISCOSITY

METHOD $=$ LETSOU-S

\begin{tabular}{|c|c|c|c|c|}
\hline \multirow{2}{*}{$\begin{array}{l}\text { POINT } \\
\text { No. }\end{array}$} & \multirow{2}{*}{$\begin{array}{l}\text { TEMP } \\
(\mathrm{K})\end{array}$} & \multicolumn{2}{|c|}{ (N-SEC/SQM ) } & \multirow[b]{2}{*}{$\%$ ERROR } \\
\hline & & DATA & CALCULATED & \\
\hline$-n$ & $---\infty$ & ---- & ---------- & \\
\hline 1 & 498.04 & $0.2365 \mathrm{E}-04$ & $0.2365 \mathrm{E}-04$ & 0.00 \\
\hline 2 & 514.80 & $0.7242 \mathrm{E}-04$ & $0.7242 \mathrm{E}-04$ & 0.00 \\
\hline 3 & 397.51 & & $0.3955 E-08$ & \\
\hline 4 & 414.27 & & $0.2259 \mathrm{E}-07$ & \\
\hline 5 & 431.02 & & $0.1127 \mathrm{E}-06$ & \\
\hline 6 & 447.78 & & $0.4983 \mathrm{E}-06$ & \\
\hline 7 & 464.53 & & $0.1980 \mathrm{E}-05$ & \\
\hline 8 & 481.29 & & $0.7144 \mathrm{E}-05$ & \\
\hline 9 & 498.04 & & $0.2365 \mathrm{E}-04$ & \\
\hline 10 & 514.80 & & $0.7242 \mathrm{E}-04$ & \\
\hline
\end{tabular}


ASPEN PLUS VER: DOS

REL: $9 \cdot 1-3$

INST: LANL-PC

$02 / 03 / 95$

PAGE 17

TANK SY-102 CLEANOUT FLOWSHEET

PROPERTY CONSTANT ESTIMATION SECTION

PURE COMPONENT PARAMETERS (CONTINUED)

TEMPERATURE-DEPENDENT PROPERTIES

TEMPERATURE-DEPENDENT PROPERTIES

PROPERTY NAME

PARAMETER

NAME (EL)

PARAMETER

VALUES

UNITS

METHOD OF

$-------0$

HEAT OF VAPORIZATION

DHVLWT

(1) $0.69793 \mathrm{E}+08$

(2) 593.15

(3) 0.38000

(4) 0 .

(5) 593.15

LIQ THERM CONDUCTIVITY KLDIP
(1) -0.89803
(2) $0.61345 \mathrm{E}-02$
(3) $-0.13096 \mathrm{E}-04$
(4) $0.12067 \mathrm{E}-07$
(5) $-0.41900 \mathrm{E}-11$
(6) 593.15
(7) 963.27

K, WATT/M-K SATO-RIEDEL 
ASPEN PLUS VER: DOS

REL: $9.1-3$

INST: LANL-PC

$02 / 03 / 95$

PAGE 18

TANK SY-102 CLEANOUT FLOWSHEET

PROPERTY CONSTANT ESTIMATION SECTION

PURE COMPONENT PARAMETERS (CONTINUED)

DEVIATIONS IN REGRESSING TEMPERATURE-DEPENDENT PROPERTIES

LIQ THERM CONDUCTIVITY

METHOD = SATO-RIE

\begin{tabular}{|c|c|c|c|c|}
\hline \multirow{3}{*}{$\begin{array}{c}\text { POINT } \\
\text { NO. }\end{array}$} & \multirow{3}{*}{$\begin{array}{l}\text { TEMP } \\
\text { (K) }\end{array}$} & \multicolumn{2}{|c|}{ (WATT/M-K ) } & \multirow[b]{3}{*}{ \% ERROR } \\
\hline & & & & \\
\hline & & DATA & CALCULATED & \\
\hline 9 & --0 & $---1--1$ & ------1 & \\
\hline 1 & 593.15 & 0.1331 & 0.1330 & -0.09 \\
\hline 2 & 634.27 & 0.1254 & 0.1257 & 0.19 \\
\hline 3 & 675.40 & 0.1175 & 0.1175 & 0.03 \\
\hline 4 & 716.52 & 0.1091 & 0.1090 & -0.17 \\
\hline 5 & 757.65 & 0.1003 & 0.1002 & -0.18 \\
\hline 6 & 798.77 & $0.9097 \mathrm{E}-01$ & $0.9101 \mathrm{E}-01$ & 0.04 \\
\hline 7 & 839.90 & $0.8081 \mathrm{E}-01$ & $0.8109 \mathrm{E}-01$ & 0.34 \\
\hline 8 & 881.02 & $0.6954 \mathrm{E}-01$ & $0.6972 \mathrm{E}-01$ & 0.26 \\
\hline 9 & 922.15 & $0.5636 \mathrm{E}-01$ & $0.5590 E-01$ & -0.82 \\
\hline 10 & 963.27 & $0.3820 \mathrm{E}-01$ & $0.3838 E-01$ & 0.46 \\
\hline
\end{tabular}


ASPEN PLUS VER: DOS

REL: $9 \cdot 1-3$

INST: LANL-PC

$02 / 03 / 95$

PAGE 19

TANK SY-102 CLEANOUT FLOWSHEET

PROPERTY CONSTANT ESTIMATION SECTION

PURE COMPONENT PARAMETERS (CONTINUED)

\begin{tabular}{lllll} 
& & & \\
POMPONENT ID: CSOH(S) & FORMULA: & & \\
PROPERTY NAME & PARAMETER & ESTIMATED & & METHOD OF \\
\hline CRITICAL TEMPERATURE & NAME & VALUE & UNITS & ESTIMATION \\
HEAT OF VAP AT TC & TC & 1807.04 & $\mathrm{~K}$ & SIMPLE \\
& DHVLB & $0.16683 \mathrm{E}+09$ & $\mathrm{~J} / \mathrm{KMOL}$ & VETERE
\end{tabular}


REL: $9.1-3$

INST: LANL-PC

PROPERTY CONSTANT ESTIMATION SECTION

PURE COMPONENT PARAMETERS (CONTINUED)

TEMPERATURE-DEPENDENT PROPERTIES

\begin{tabular}{|c|c|c|c|c|c|}
\hline PROPERTY NAME & \multicolumn{2}{|c|}{$\begin{array}{l}\text { PARAMETER } \\
\text { NAME (EL) }\end{array}$} & $\begin{array}{l}\text { PARAMETER } \\
\text { VALUES }\end{array}$ & UNITS & $\begin{array}{l}\text { METHOD OF } \\
\text { ESTIMATION }\end{array}$ \\
\hline - - - - - - - & $--\cdots$ & & $---\cdots-\cdots$ & $--\infty$ & $-\cdots-\cdots-\cdots$ \\
\hline HEAT OF VAPORIZATION & DHVLWT & $\begin{array}{l}(1) \\
(2) \\
(3) \\
(4) \\
(5)\end{array}$ & $\begin{array}{c}0.16683 \mathrm{E}+09 \\
1263.0 \\
0.38000 \\
0 . \\
1263.0\end{array}$ & $\mathrm{~K}, \mathrm{~J} / \mathrm{KMOL}$ & VETERE \\
\hline LIQ THERM CONDUCTIVITY & KLDIP & $\begin{array}{l}(1) \\
(2) \\
(3) \\
(4) \\
(5) \\
(6) \\
(7)\end{array}$ & $\begin{array}{r}-2.3573 \\
0.69769 \mathrm{E}-02 \\
-0.73220 \mathrm{E}-05 \\
0.33793 \mathrm{E}-08 \\
-0.58667 \mathrm{E}-12 \\
1263.0 \\
1789.0\end{array}$ & $\mathrm{~K}, \mathrm{WATT} / \mathrm{M}-\mathrm{K}$ & SATO-RIEDEI \\
\hline
\end{tabular}


ASPEN PLUS VER: LOS

REL: $9.1-3$

INST: LANL-PC

$02 / 03 / 95$

PAGE 21

TANK SY-102 CLEANOUT FLOWSHEET

PROPERTY CONSTANT ESTIMATION SECTION

PURE COMPONENT PARAMETERS (CONTINUED)

DEVIATIONS IN REGRESSING TEMPERATURE-DEPENDENT PROPERTIES

LIQ THERM CONDUCTIVITY

METHOD = SATO-RIE

\begin{tabular}{|c|c|c|c|c|}
\hline \multirow{3}{*}{$\begin{array}{l}\text { POINT } \\
\text { NO. }\end{array}$} & \multirow{2}{*}{$\begin{array}{l}\text { TEMP } \\
(\mathrm{K})\end{array}$} & \multicolumn{2}{|c|}{ (WATT/M-K ) } & \multirow{3}{*}{$\%$ ERROR } \\
\hline & & DATA & CALCULATED & \\
\hline & $--\infty$ & ------- & -ー-ー-ー-ー- & \\
\hline 1 & 1263.00 & $0.9027 \mathrm{E}-01$ & $0.9021 E-01$ & -0.07 \\
\hline 2 & 1321.44 & $0.8534 \mathrm{E}-01$ & $0.8547 \mathrm{E}-0 \mathrm{I}$ & 0.15 \\
\hline 3 & 1379.88 & $0.8020 E-01$ & $0.8022 \mathrm{E}-01$ & 0.03 \\
\hline 4 & 1438.32 & $0.7482 \mathrm{E}-01$ & $0.7471 E-01$ & -0.14 \\
\hline 5 & 1496.77 & $0.6914 \mathrm{E}-01$ & $0.6904 \mathrm{E}-01$ & -0.15 \\
\hline 6 & 1555.21 & $0.6310 \mathrm{E}-01$ & $0.6312 E-01$ & 0.04 \\
\hline 7 & 1613.65 & $0.5656 \mathrm{E}-01$ & $0.5671 \mathrm{E}-01$ & 0.27 \\
\hline 8 & 1672.09 & $0.4932 \mathrm{E}-01$ & $0.4941 \mathrm{E}-01$ & 0.19 \\
\hline 9 & 1730.53 & $0.4090 \mathrm{E}-01$ & $0.4065 E-01$ & -0.62 \\
\hline 10 & 1788.97 & $0.2959 E-01$ & $0.2969 E-01$ & 0.32 \\
\hline
\end{tabular}


ASPEN PLUS VER: DOS

REL: $9.1-3$ INST: I.ANL-PC

TANK SY-102 CLEANOUT FLOWSHEET

U-O-S BLOCK SECTION

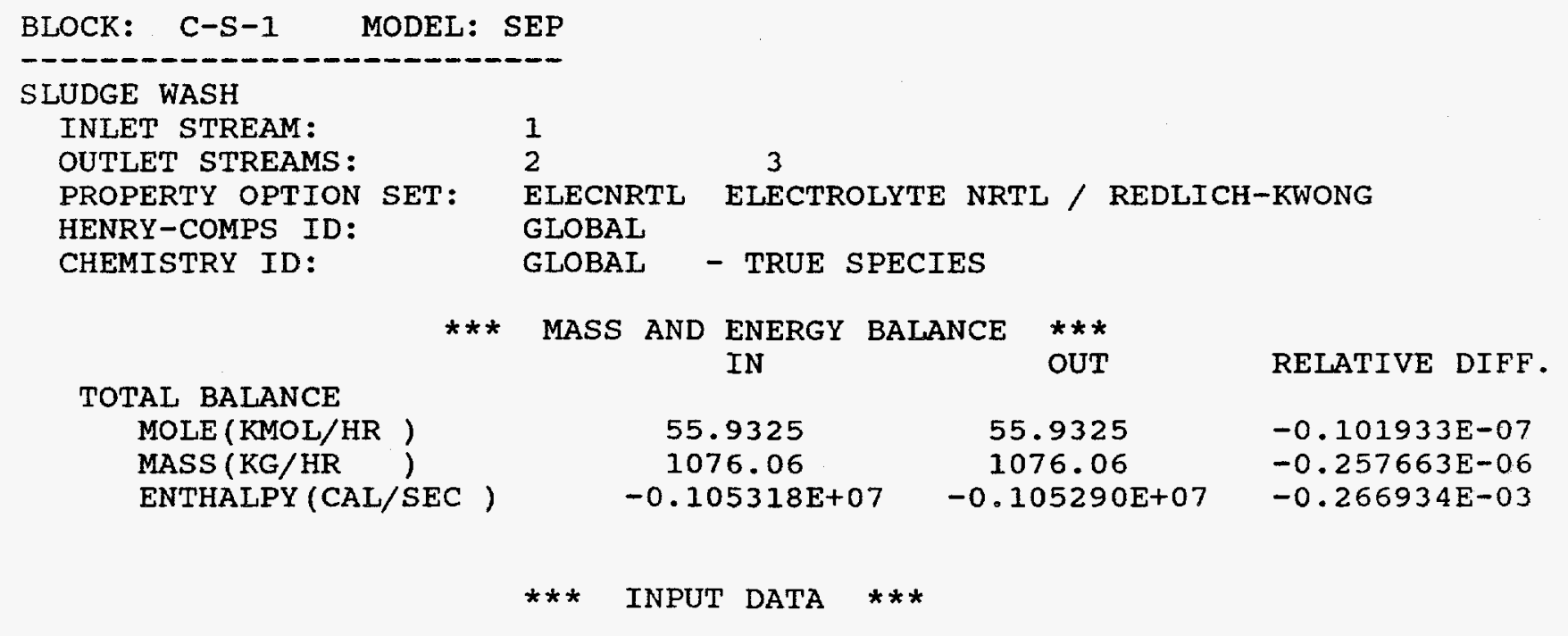

FLASH SPECS FOR STREAM 2

TWO PHASE TP FLASH

PRESSURE DROP ATM

MAXIMUM NO. ITERATIONS

CONVERGENCE TOLERANCE

0.0

30

0.000100000

FLASH SPECS FOR STREAM 3

TWO PHASE TP FLASH

PRESSURE DROP ATM

MAXIMUM NO. ITERATIONS

CONVERGENCE TOLERANCE

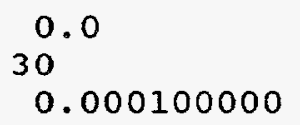


ASPEN PLUS VER: DOS

REL: $9.1-3$

INST: LANL-PC

$02 / 03 / 95$

PAGE 23

TANK SY-102 CLEANOUT FLOWSHEET

U-O-S BLOCK SECTION

BLOCK: C-S-1 MODEL: SEP (CONTINUED)

FRACTION OF FEED

SUBSTREAM $=$ MIXED

STREAM $=2$

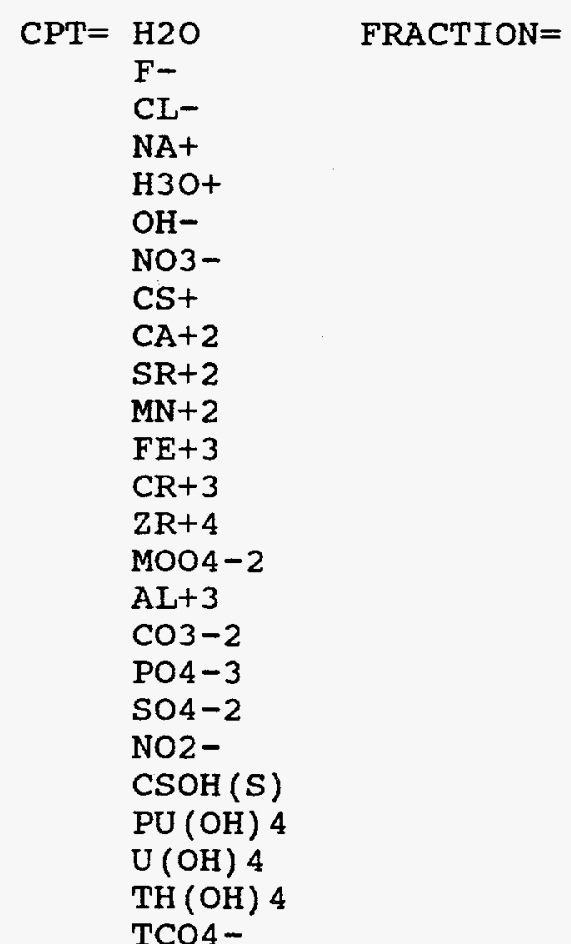

0.83950

0.40140

1.00000

0.99000

0.0

0.56440

1.00000

0.90000

0.0

0.0

0.0

0.0

0.0

0.0

0.0

0.13780

0.29410

1.00000

0.28660

1.00000

0.90000

0.0

0.0

0.0

$\mathrm{TCO} 4$ -

0.99990
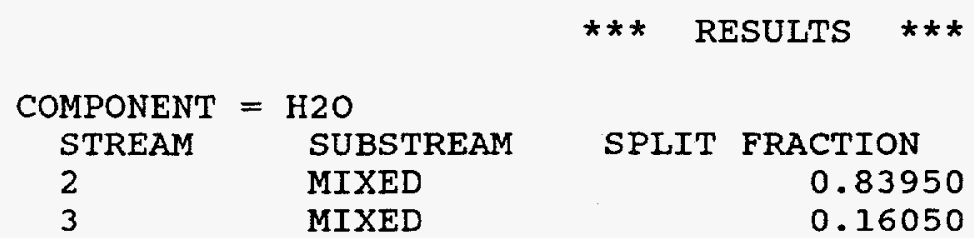

SPLIT FRACTION

0.83950

0.16050

COMPONENT $=\mathrm{F}-$

STREAM SUBSTREAM

2 MIXED

3 MIXED

SPLIT FRACTION

0.40140

0.59860

COMPONENT $=\mathrm{CL}-$ STREAM SUBSTREAM

2 MIXED

SPLIT FRACTION

1.00000

COMPONENT $=$ NAt

STREAM SUBSTREAM

2 MIXED

3 MIXED

SPIIT FRACTION

0.99000

0.0100000 
BLOCK: C-S-1 MODEL: SEP (CONTINUED)

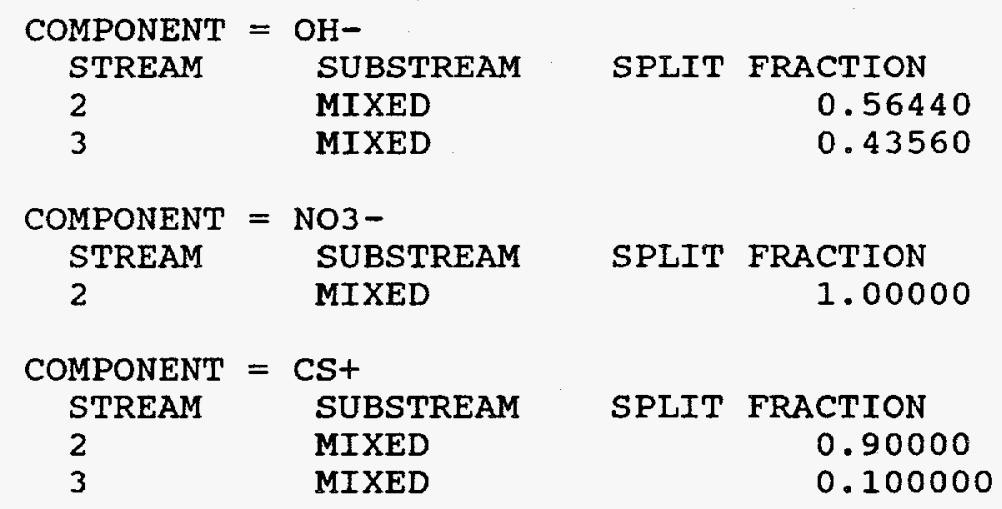


ASPEN PLUS VER: DOS

REL: 9.1-3 INST: LANL-PC

TANK SY-102 CLLEANOUT FLOWSHEET

U-O-S BLOCK SECTION

BLOCK: C-S-1 MODEL: SEP (CONTINUED)

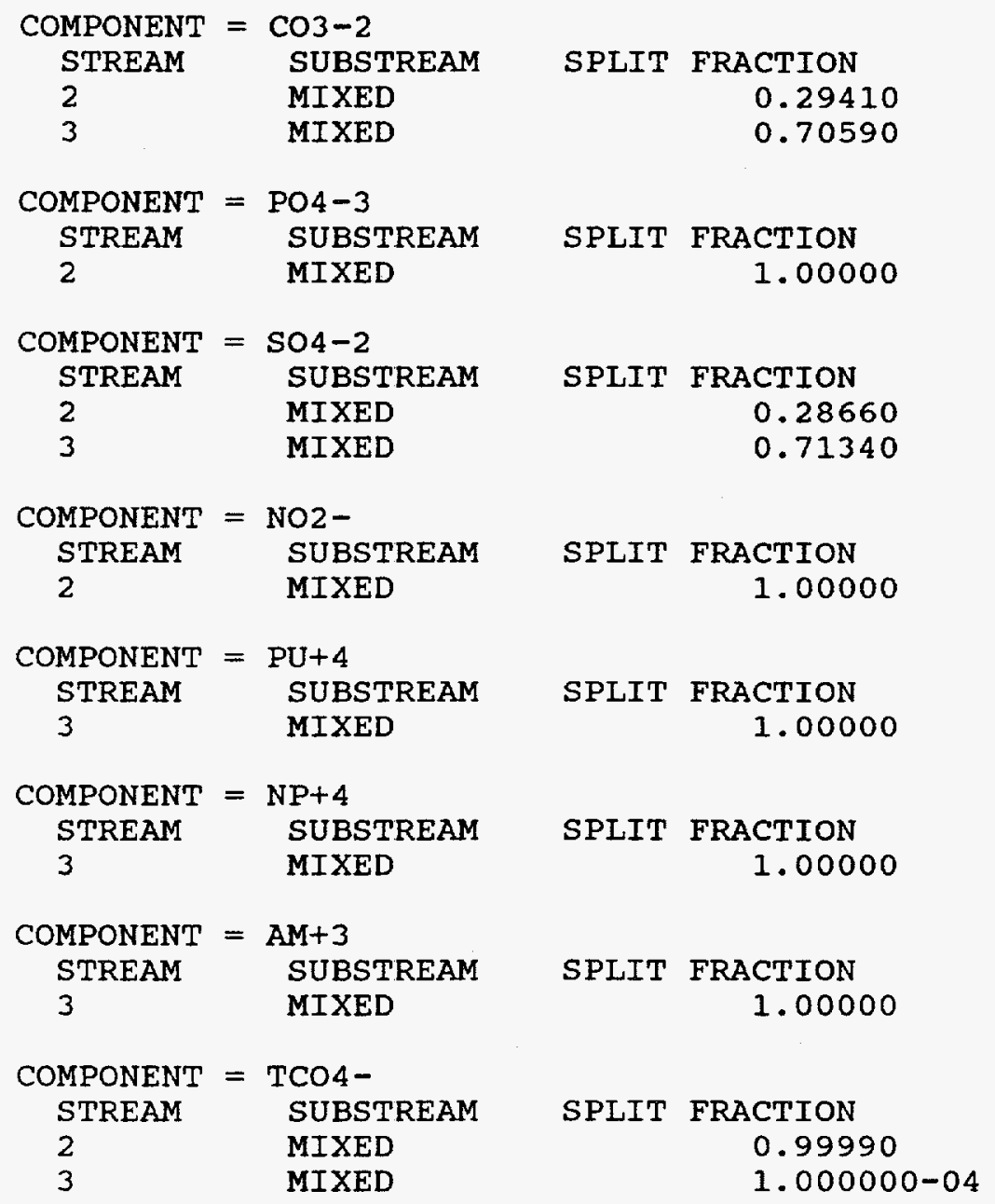




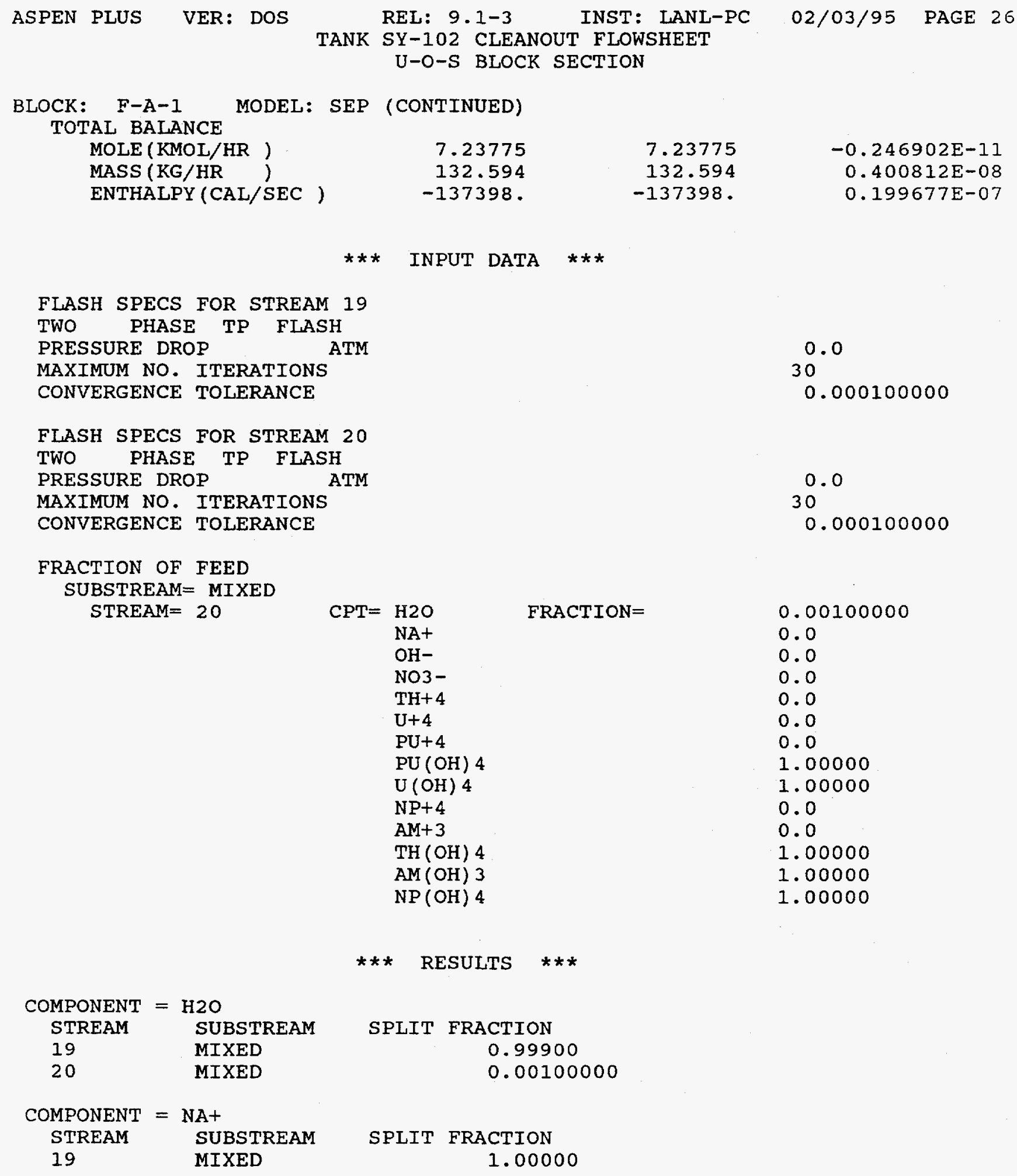




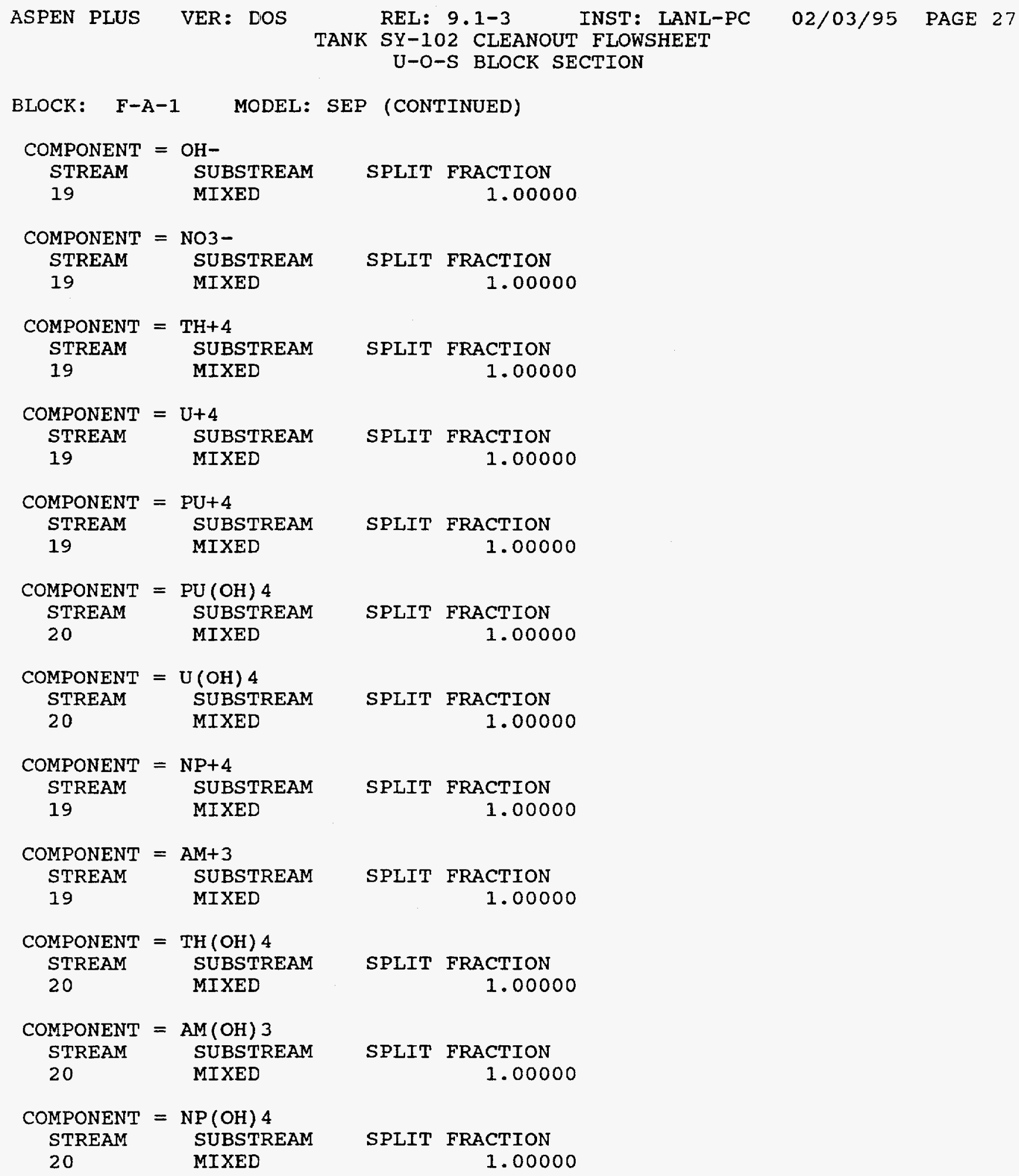


TANK SY-102 CLEANOUT FLOWSHEET

U-O-S BLOCK SECTION

BLOCK: IX-A-1 MODEL: SEP

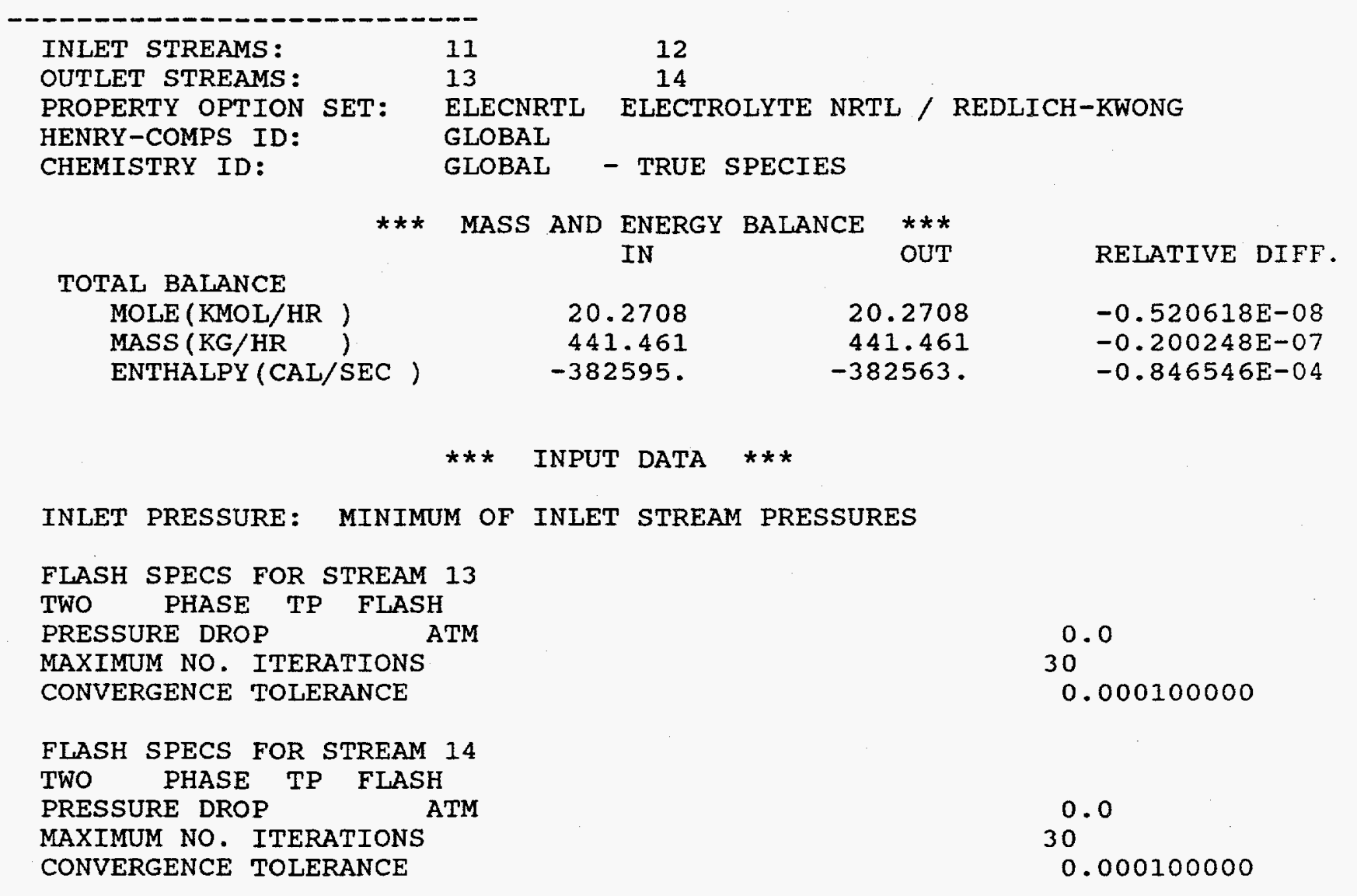




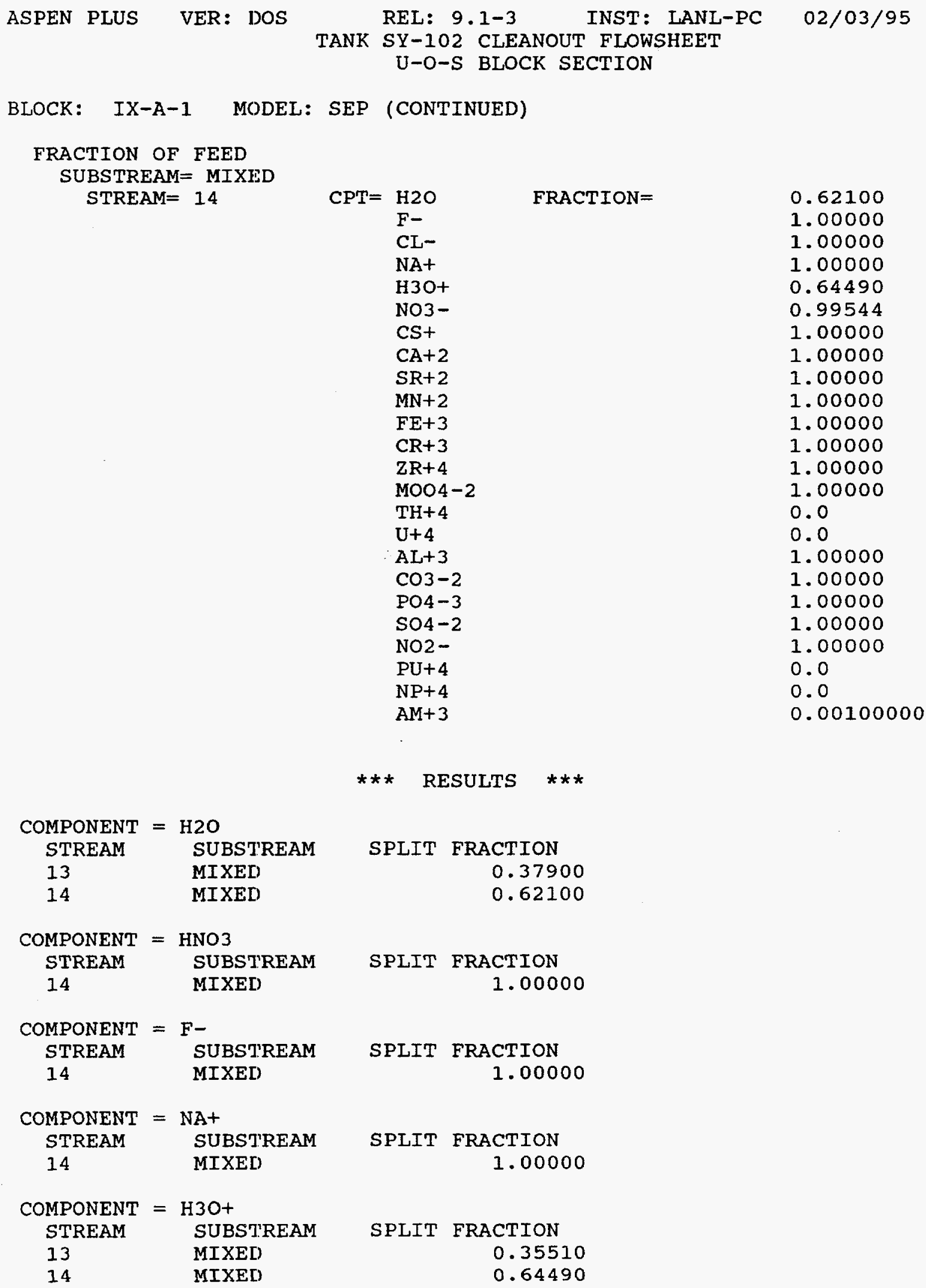




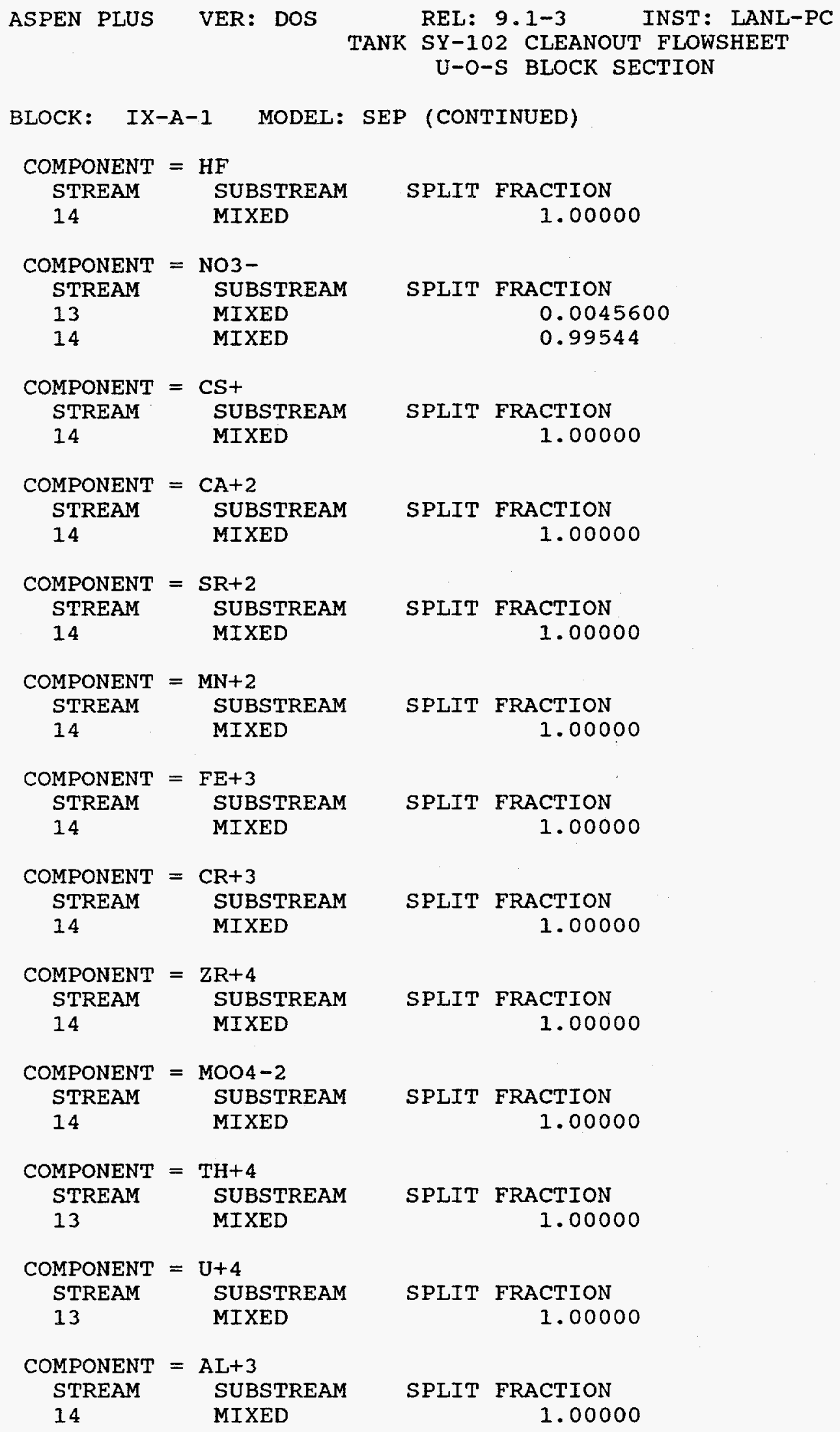




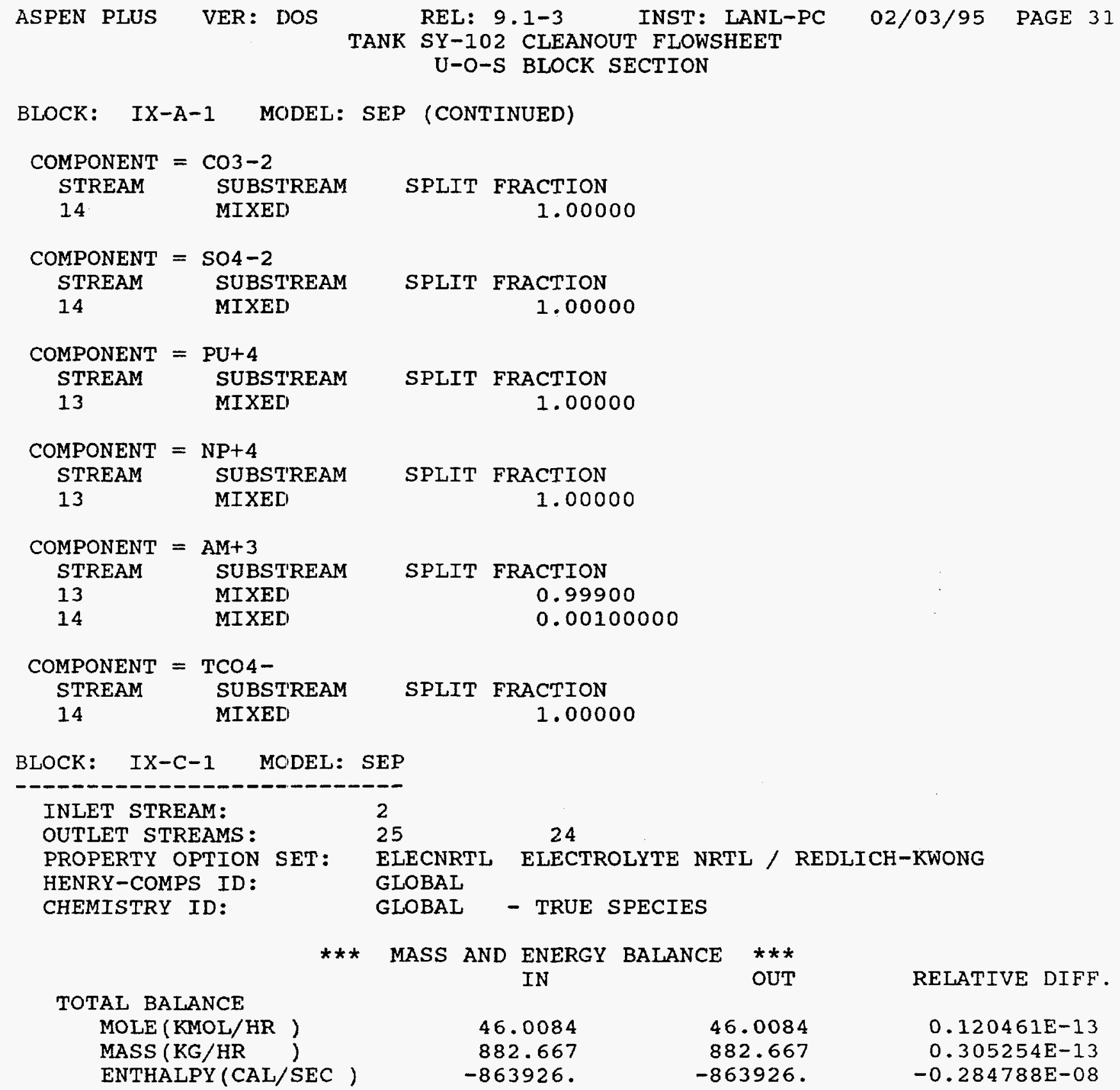


ASPEN PLUS VER: DOS

REL: 9.1-3 INST: LANL-PC

02/03/95 PAGE 32

TANK SY-102 CLEANOUT FLOWSHEET

U-O-S BLOCK SECTION

BLOCK: IX-C-1 MODEL: SEP (CONTINUED)

$$
\text { *** INPUT DATA } * * *
$$

FLASH SPECS FOR STREAM 25

TWO PHASE TP FLASH

PRESSURE DROP ATM

MAXIMUM NO. ITERATIONS

CONVERGENCE TOLERANCE

0.0

30

0.000100000

FLASH SPECS FOR STREAM 24

TWO PHASE TP FLASH

PRESSURE DROP ATM

MAXIMUM NO. ITERATIONS

CONVERGENCE TOLERANCE

0.0

30

0.000100000 

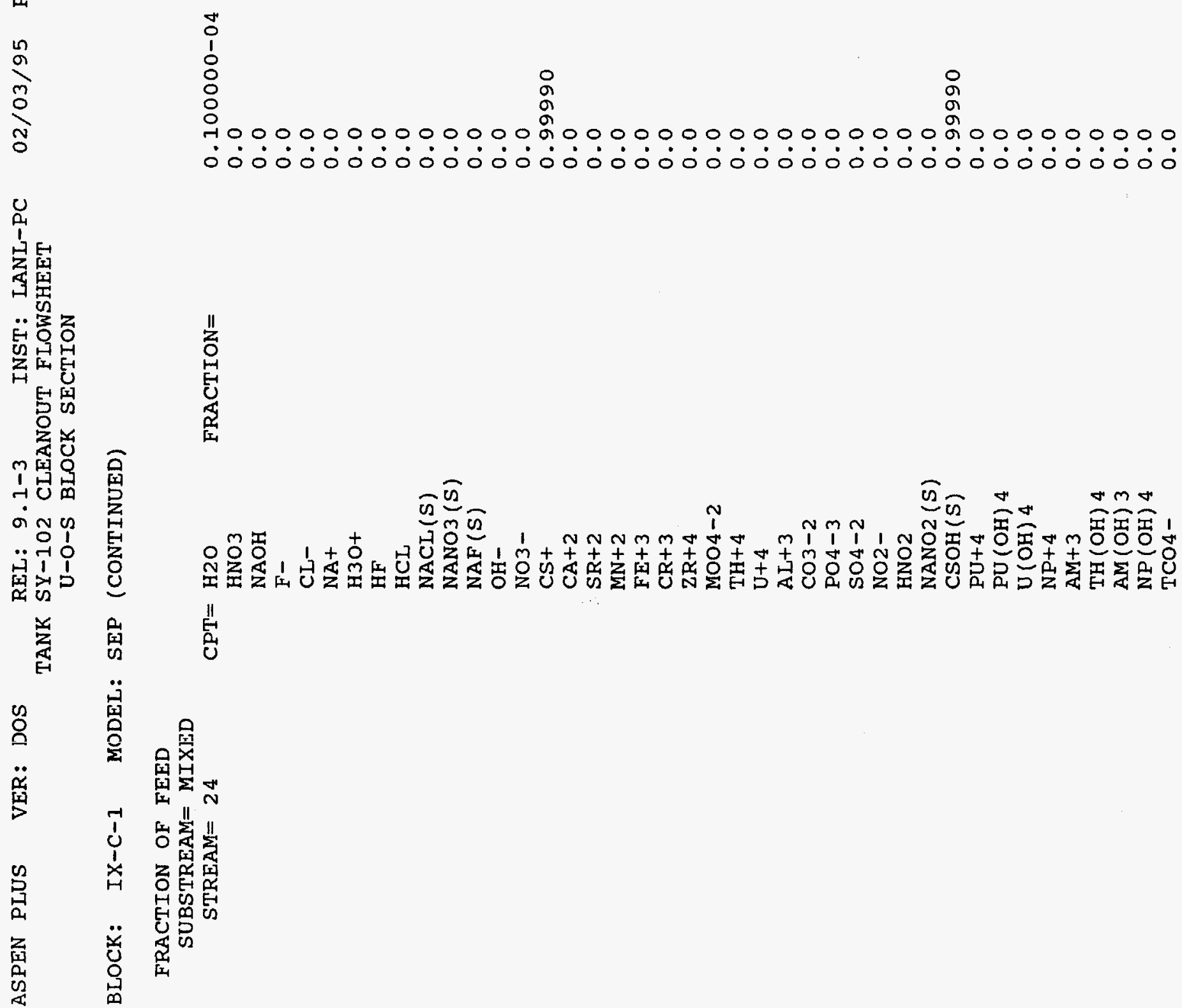

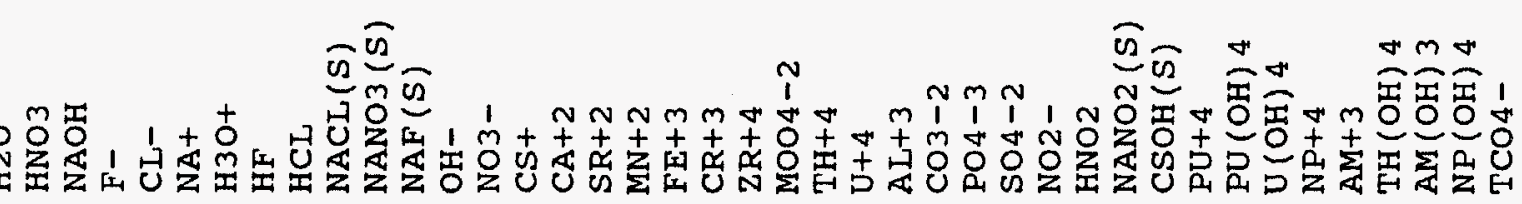
至

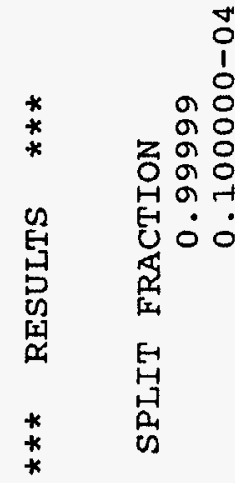

in

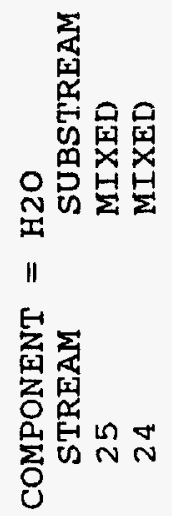




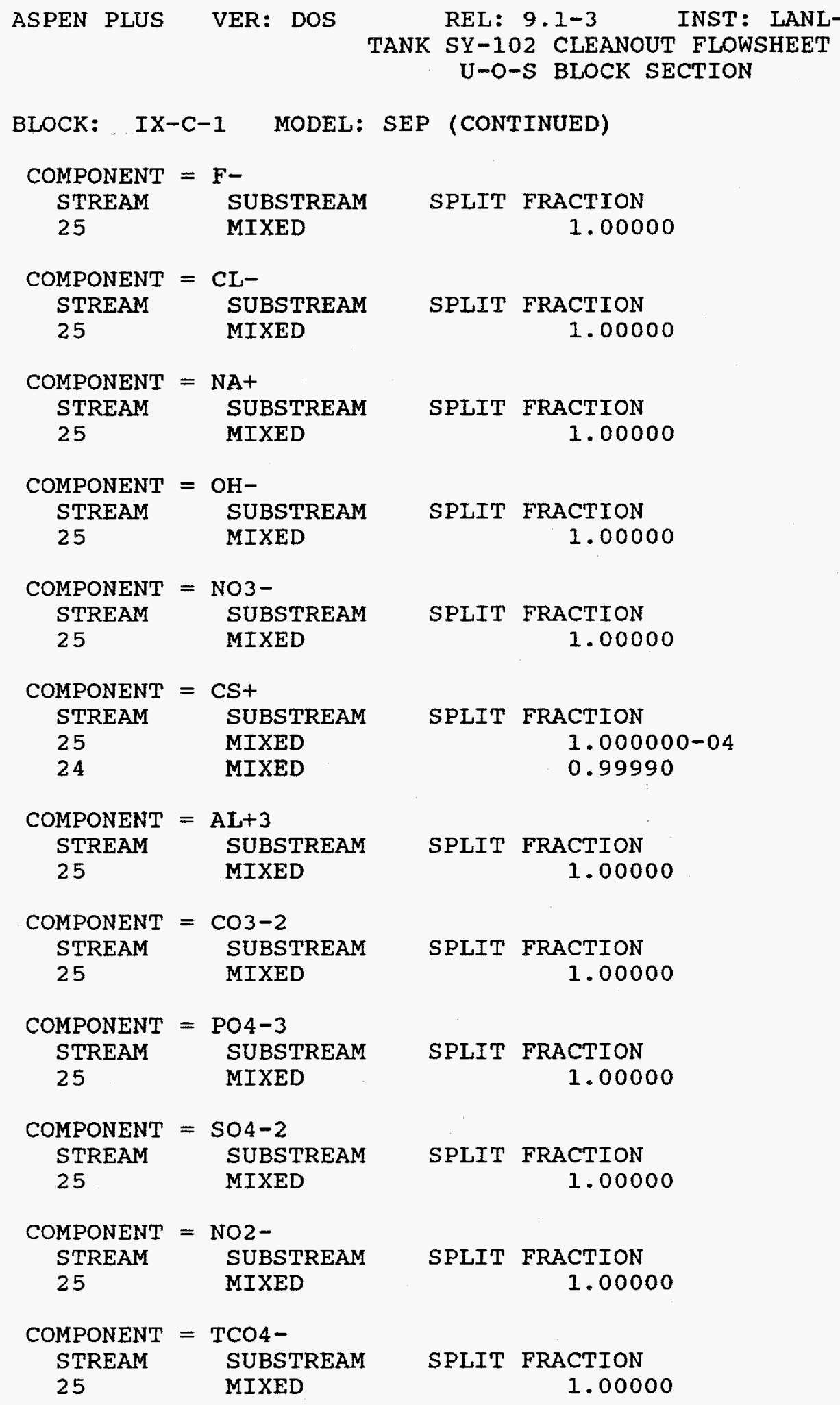


BLOCK: IX-S-1 MODEL: SEP

INLET STREAM: OUTLET STREAMS : PROPERTY OPTION SSET: HENRY-COMPS ID: CHEMISTRY ID:

\section{4}

$15 \quad 16$

ELECNRTL ELECTROLYTE NRTL / REDLICH-KWONG GLOBAL GLOBAL

- TRUE SPECIES

$$
\star * *
$$

\section{TOTAL BALANCE}

MOLE (KMOL/HR )

MASS (KG/HR

ENTHALPY (CAL/SEC )

\section{AND ENERGY BALANCE} IN

$$
\begin{array}{r}
13.3355 \\
314.926 \\
-250967 .
\end{array}
$$

*** INPUT DATA ***
FLASH SPECS FOR STREAM 15 TWO PHASE TP FLASH PRESSURE DROP MAXIMUM NO. ITERATIONS CONVERGENCE TOLERANCE

FLASH SPECS FOR STREAM 16 TWO PHASE TP FLASH PRESSURE DROP ATM MAXIMUM NO. ITERATIONS CONVERGENCE TOLEEANCE ATM

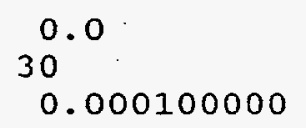

13.3355

314.926

-250967 .

RELATIVE DIFF.
$-0.265604 \mathrm{E}-07$

$-0.234987 \mathrm{E}-07$

$0.224232 \mathrm{E}-07$
0.0
30
0.000100000 


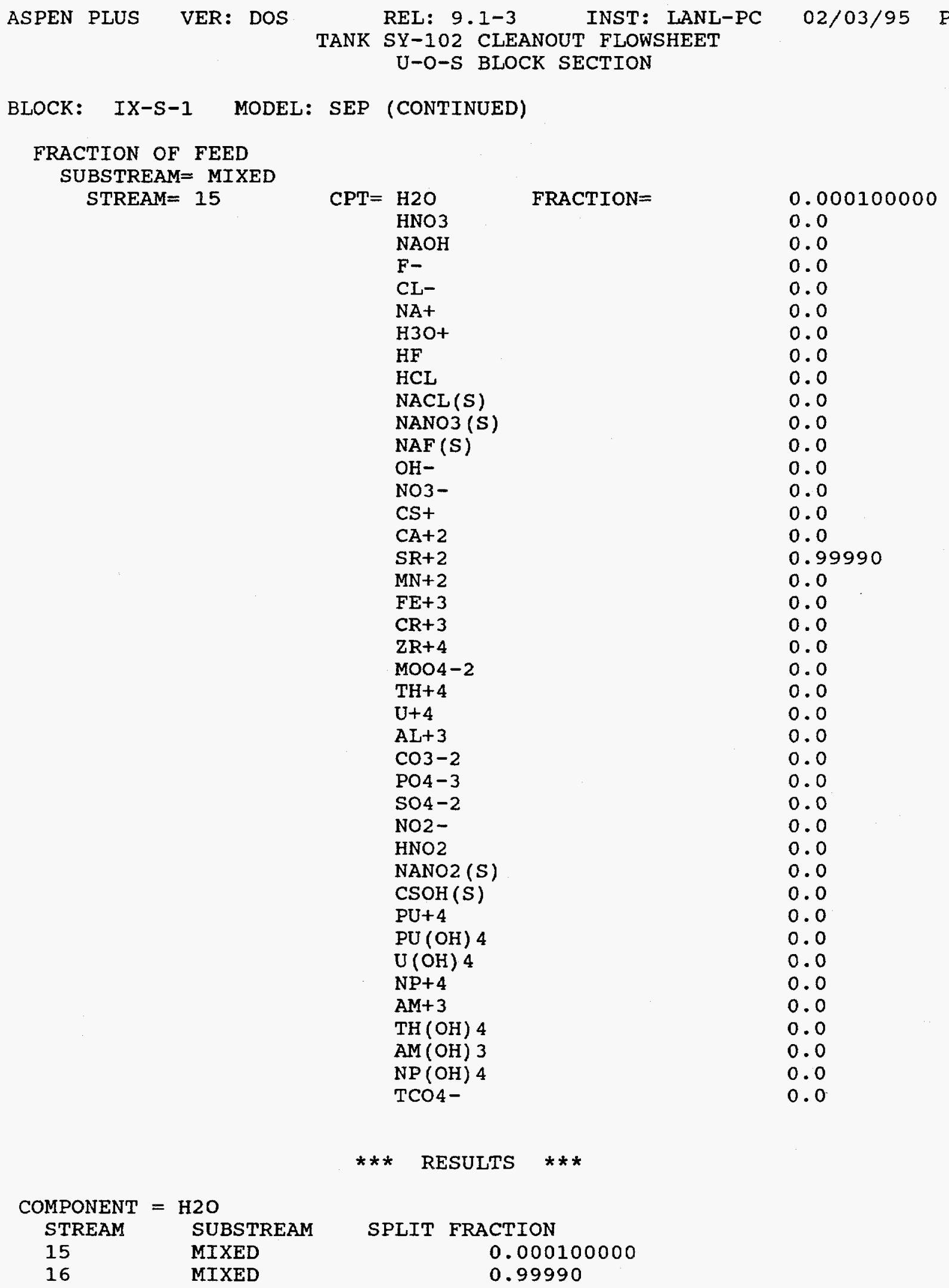




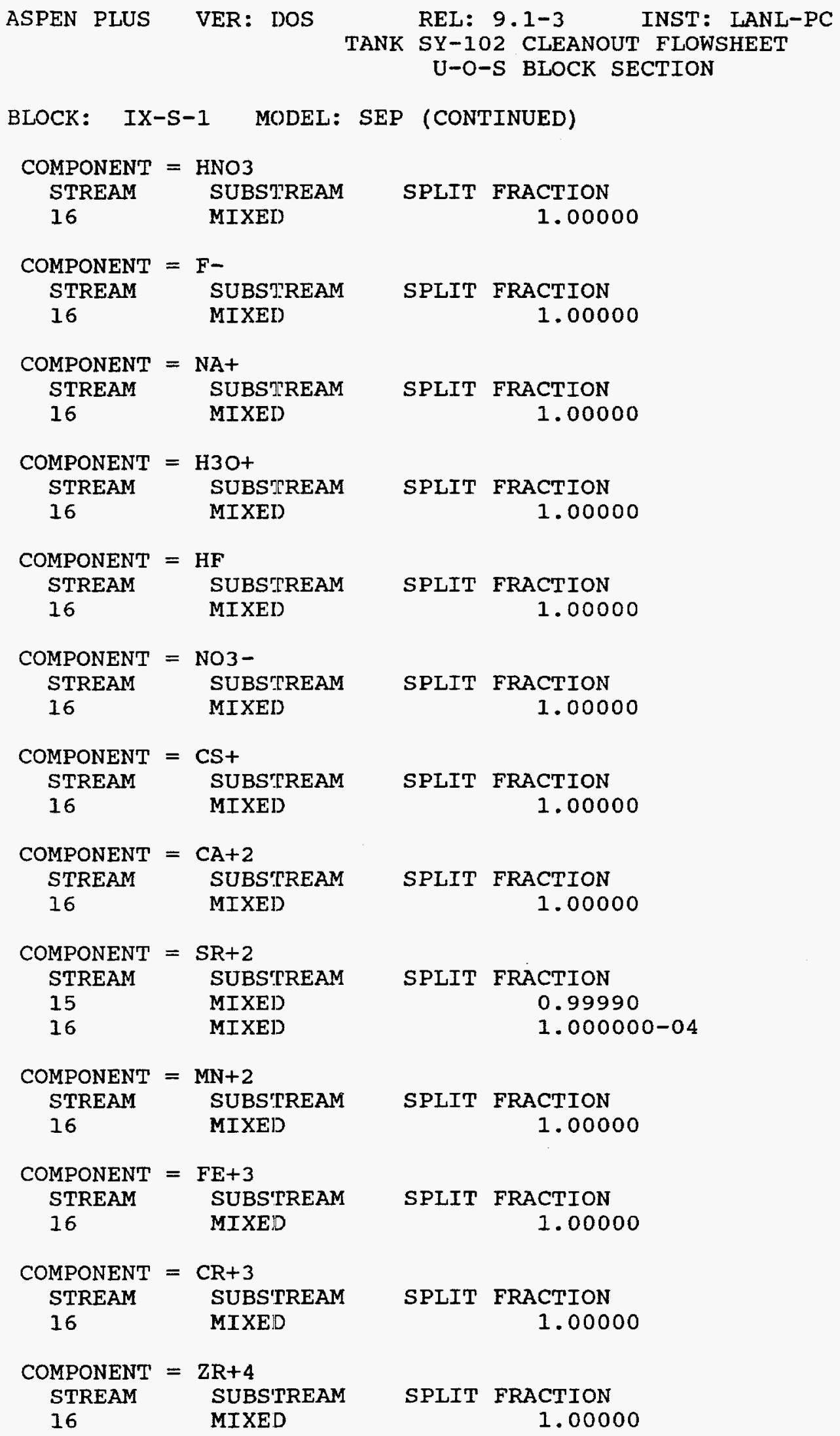




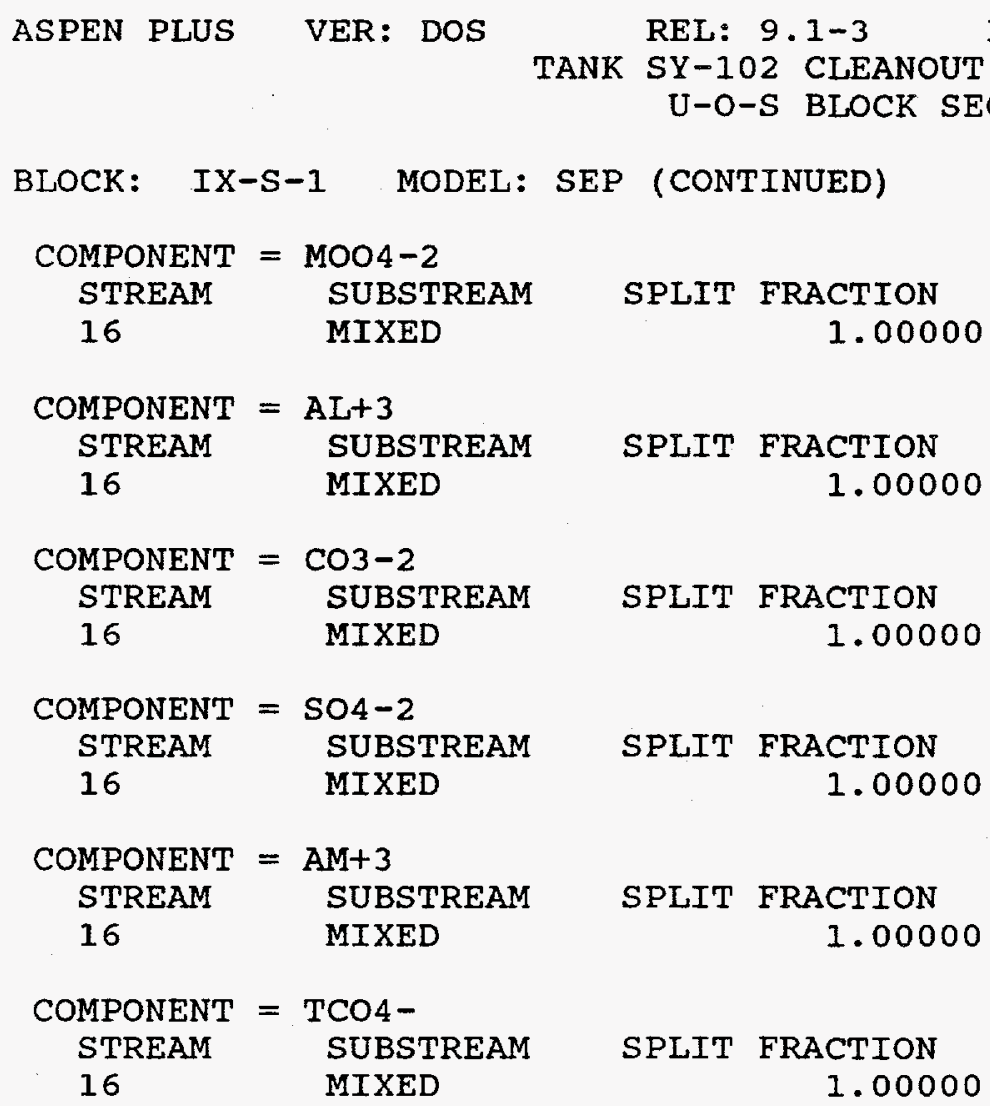


ASPEN PLUS VER: DOS

REL: $9 \cdot 1-3$

INST: LANL-PC

$02 / 03 / 95$

PAGE 39

TANK SY-102 CLEANOUT FLOWSHEET

U-O-S BLOCK SECTION

BLOCK: IX-T-1 MODEL: SEP (CONTINUED)

\section{*** INPUT DATA $* * *$}

FLASH SPECS FOR STREAM 26

TWO PHASE TP FLASH

PRESSURE DROP ATM

MAXIMUM NO. ITERATIONS

CONVERGENCE TOLEJRANCE

0.0
30
0.000100000

FLASH SPECS FOR STREAM 27

TWO PHASE TP FLASH

PRESSURE DROP ATM

MAXIMUM NO. ITERATIONS

CONVERGENCE TOLE]RANCE
0.0

30

0.000100000 


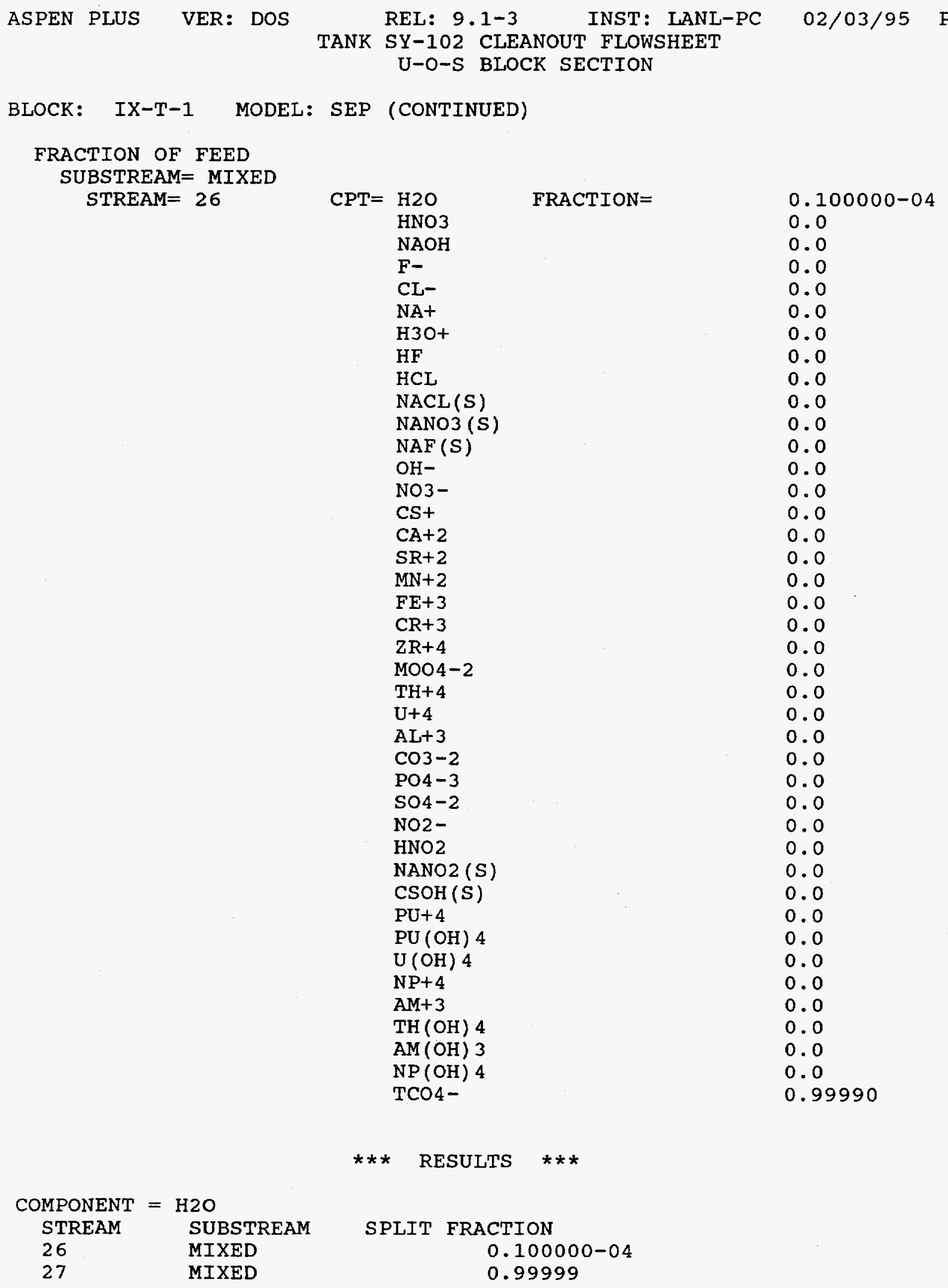


ASPEN PIUS VER: DOS

REL: $9.1-3$ INST: LANL-PC

02/03/95 PAGE 41

TANK SY-102 CLEANOUT FLOWSHEET

U-O-S BLOCK SECTION

BLOCK: IX-T-I MODEL: SEP (CONTINUED)

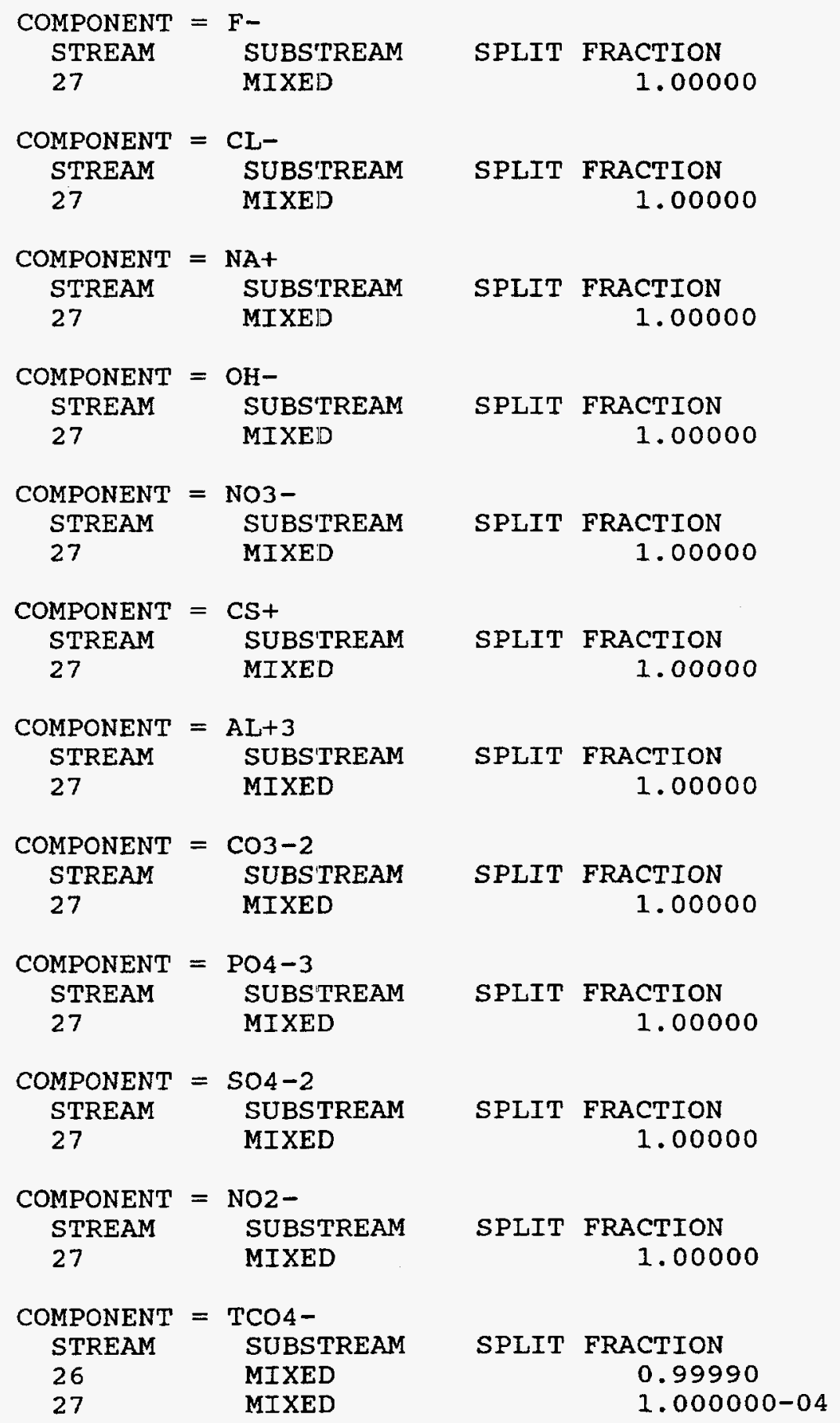




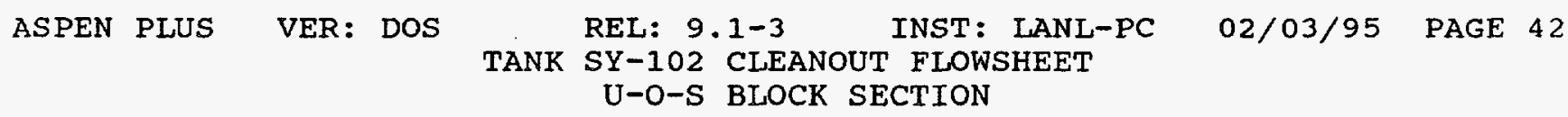

BLOCK: RC-A-1 MODEL: RSTOIC

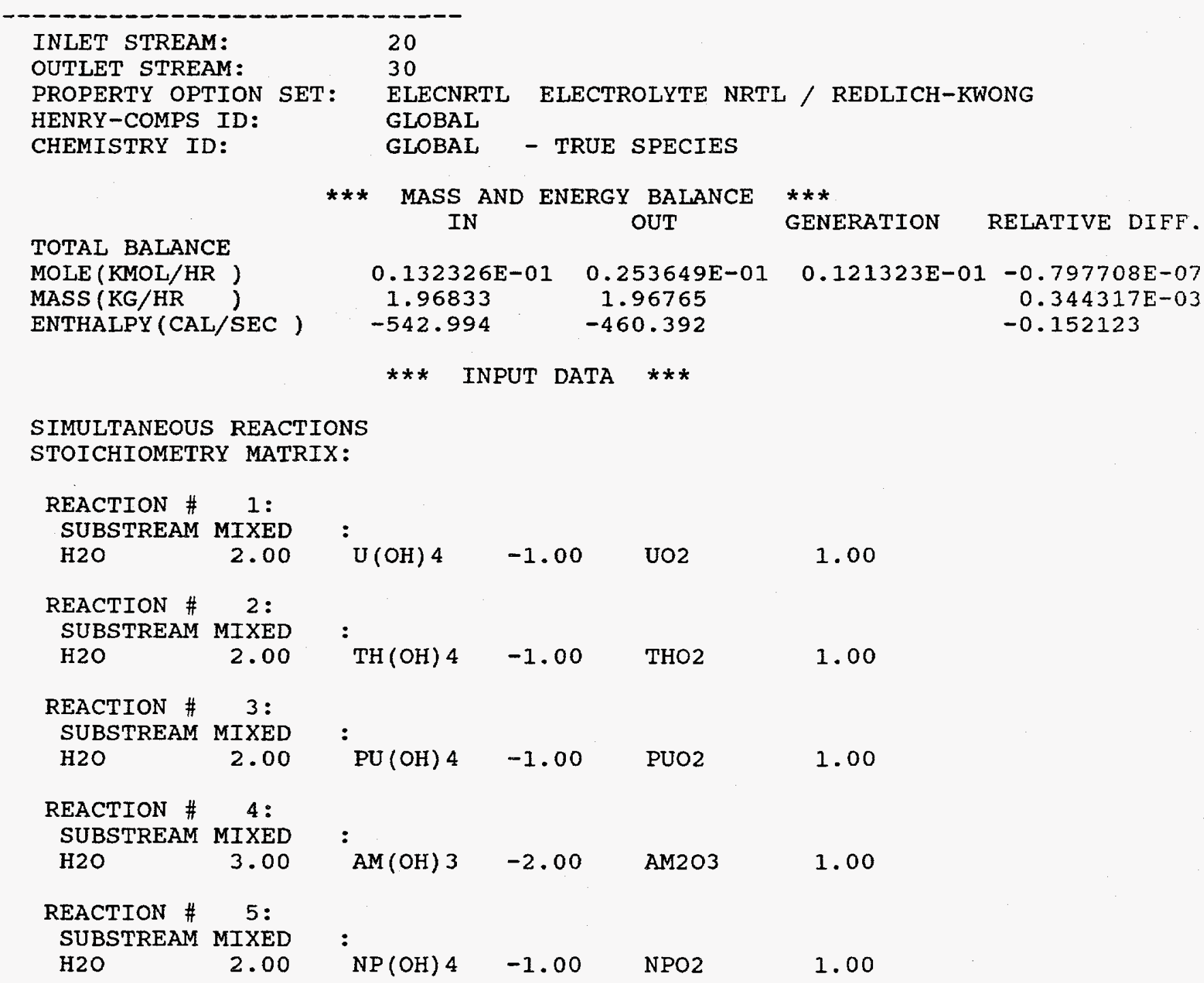


REL: $9 \cdot 1-3$

INST: LANL-PC

$02 / 03 / 95$

PAGE 43

TANK SY-102 CLEANOUT FLOWSHEET

U-O-S BLOCK SECTION

BLOCK: RC-A-1 MODEL: RSTOIC (CONTINUED)

REACTION CONVERSION SPECS: NUMBER=

REACTION \# 1:

SUBSTREAM:MIXED KEY COMP:U(OH) 4 CONV FRAC: 1.000

REACTION \# 2:

SUBSTREAM : MIXED

REACTION \# 3:

SUBSTREAM : MIXED

REACTION \# 4 :

SUBSTREAM : MIXED

REACTION \# 5:

KEY COMP:TH(OH) 4 CONV FRAC: 1.000

KEY COMP: PU(OH) 4 CONV FRAC: 1.000

KEY COMP:AM(OH) 3 CONV FRAC: 1.000

SUBSTREAM : MIXED

KEY COMP:NP(OH) 4 CONV FRAC: 1.000

TWO PHASE TP FLASH

SPECIFIED TEMPERATURE $K$

SPECIFIED PRESSURE ATM

MAXIMUM NO. ITERATIONS

CONVERGENCE TOLERANCE

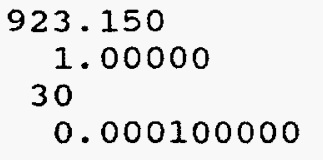

\begin{tabular}{|c|c|}
\hline IPERATURE & RESULTS \\
\hline OUTLET PRESSURE & ATM \\
\hline HEAT DUTY & $\mathrm{CAL} / \mathrm{SEC}$ \\
\hline
\end{tabular}

923.15

1.0000

82.415

VAPOR FRACTION

0.75993

V-L PHASE EQUILIBRIUM :

$\begin{array}{llllc}\text { COMP } & F(I) & X(I) & Y(I) & K(I) \\ \text { H2O } & 0.76084 & 0.38277 \mathrm{E}-02 & 1.0000 & 261.25 \\ \text { H3O+ } & 0.00000 \mathrm{E}+00 & 0.22367 \mathrm{E}-20 & 0.00000 \mathrm{E}+00 & 0.10009 \mathrm{E}-35 \\ \text { OH- } & 0.69222 \mathrm{E}-09 & 0.33516 \mathrm{E}-06 & 0.00000 \mathrm{E}+00 & 0.10009 \mathrm{E}-35 \\ \text { PUO2 } & 0.27191 \mathrm{E}-02 & 0.11326 \mathrm{E}-01 & 0.00000 \mathrm{E}+00 & 0.51319 \mathrm{E}-21 \\ \mathrm{UO2} & 0.11814 & 0.49210 & 0.13417 \mathrm{E}-14 & 0.27265 \mathrm{E}-14 \\ \text { AM2O3 } & 0.10938 \mathrm{E}-03 & 0.45563 \mathrm{E}-03 & 0.00000 \mathrm{E}+00 & 0.10009 \mathrm{E}-35 \\ \text { NPO2 } & 0.49688 \mathrm{E}-04 & 0.20697 \mathrm{E}-03 & 0.00000 \mathrm{E}+00 & 0.10009 \mathrm{E}-35 \\ \text { THO2 } & 0.11814 & 0.49208 & 0.00000 \mathrm{E}+00 & 0.10009 \mathrm{E}-35\end{array}$


TANK SY-102 CLEANOUT FLOWSHEET

U-O-S BLOCK SECTION

BLOCK: SPLIT MODEL: FLASH2

INLET STREAM: 30

OUTLET VAPOR STREAM: 21

OUTLET LIQUID STREAM: 22

PROPERTY OPTION SET: ELECNRTL ELECTROLYTE NRTL / REDLICH-KWONG

HENRY-COMPS ID:

CHEMISTRY ID:

GLOBAL

GLOBAL - TRUE SPECIES
*** MASS AND ENERGY BALANCE ***

IN

OUT
RELATIVE DIFF.

$$
\begin{aligned}
& 0.253649 E-01 \\
& 1.96765 \\
& -460.392
\end{aligned}
$$

$0.109425 \mathrm{E}-14$

$-0.174896 \mathrm{E}-07$

$0.966028 \mathrm{E}-10$

\footnotetext{
$\star * *$ INPUT DATA $* * *$

TWO PHASE TP FLASH

SPECIFIED TEMPERATURE $K$

SPECIFIED PRESSURE ATM

MAXIMUM NO. ITERATIONS

CONVERGENCE TOLERANCE

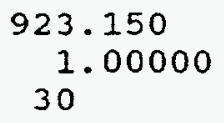

923.15

1.0000

$0.80076 \mathrm{E}-05$

0.75993
} 
ASPEN PLUS VER: DOS

REL: $9.1-3$ INST: LANL-PC

TANK SY-102 CLEANOUT FLOWSHEET

$\mathrm{U}-\mathrm{O}-\mathrm{S}$ BLOCK SECTION

BLOCK: SPLIT MODEL: FLASH2 (CONTINUED)

V-L PHASE EQUILIBRIUM :

$\begin{array}{lll}\text { COMP } & F(I) & X(I) \\ \text { H2O } & 0.76084 & 0.38277 \mathrm{E}-02 \\ \text { H3O+ } & 0.00000 \mathrm{E}+00 & 0.22377 \mathrm{E}-20 \\ \text { OH- } & 0.80464 \mathrm{E}-07 & 0.33516 \mathrm{E}-06 \\ \text { PUO2 } & 0.27191 \mathrm{E}-02 & 0.11326 \mathrm{E}-01 \\ \mathrm{UO2} & 0.11814 & 0.49210 \\ \text { AM2O3 } & 0.10938 \mathrm{E}-03 & 0.45563 \mathrm{E}-03 \\ \text { NPO2 } & 0.49688 \mathrm{E}-04 & 0.20697 \mathrm{E}-03 \\ \text { THO2 } & 0.11814 & 0.49208\end{array}$

$Y(I)$

1.0000

$0.00000 E+00$

$0.00000 E+00$

$0.00000 E+00$

$0.13417 \mathrm{E}-14$

$0.00000 \mathrm{E}+00$

$0.00000 E+00$

$0.00000 \mathrm{E}+00$
$\mathrm{K}(\mathrm{I})$

261.25

$0.10009 E-35$

$0.10009 \mathrm{E}-35$

$0.51319 \mathrm{E}-21$

$0.27265 E-14$

$0.10009 \mathrm{E}-35$

$0.10009 E-35$

$0.10009 E-35$

BLOCK : TK-G-1 MODEL: MIXER

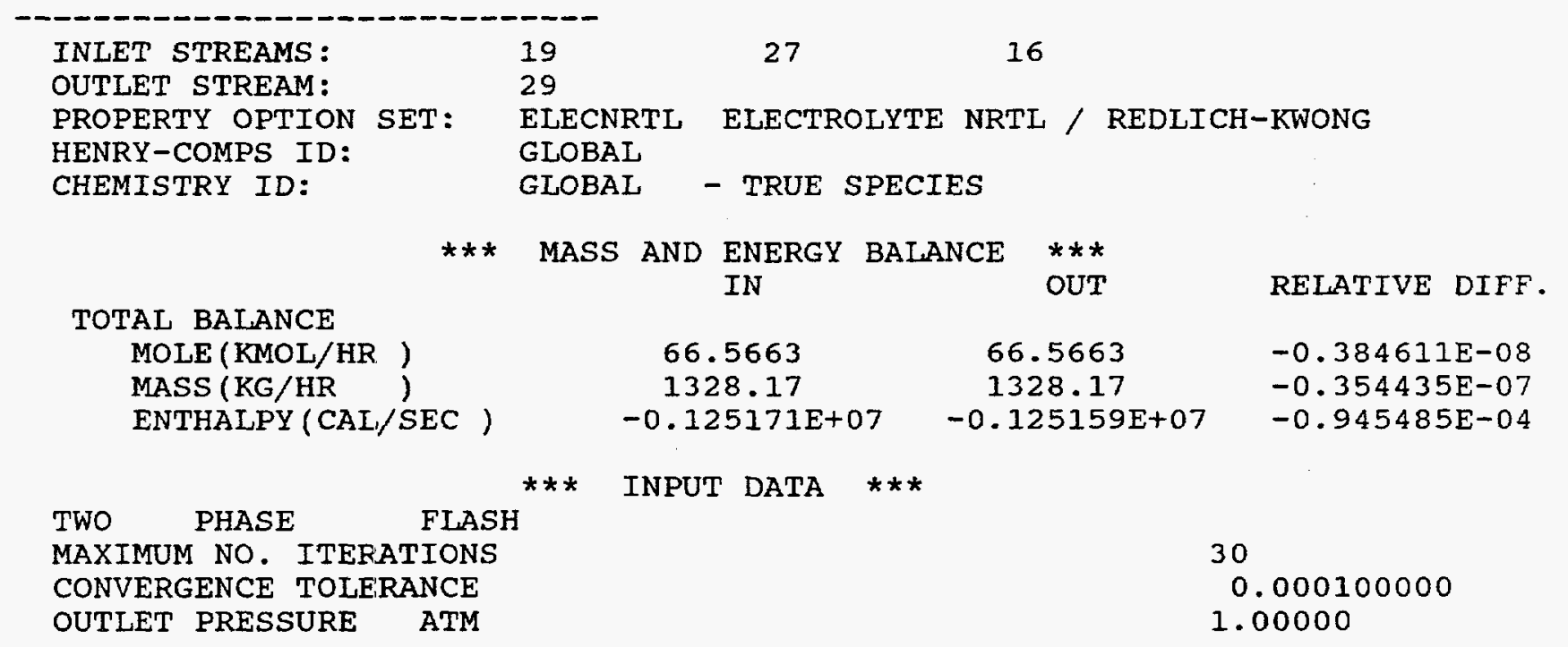

BLOCK: TK-P-1 MODEL: RSTOIC

\section{PRECIPITATION}

INLET STREAMS :

OUTLET STREAM:

PROPERTY OPTION SET:

HENRY-COMPS ID:

CHEMISTRY ID:

13

18
ELECNRTL ELECTROLYTE NRTL / REDLICH-KWONG GLOBAL

GLOBAL - TRUE SPECIES 
ASPEN PLUS VER: DOS

REL: $9.1-3$ INST: LANL-PC

TANK SY-102 CLEANOUT FLOWSHEET

U-O-S BLOCK SECTION

BLOCK: TK-P-1 MODEL: RSTOIC (CONTINUED)

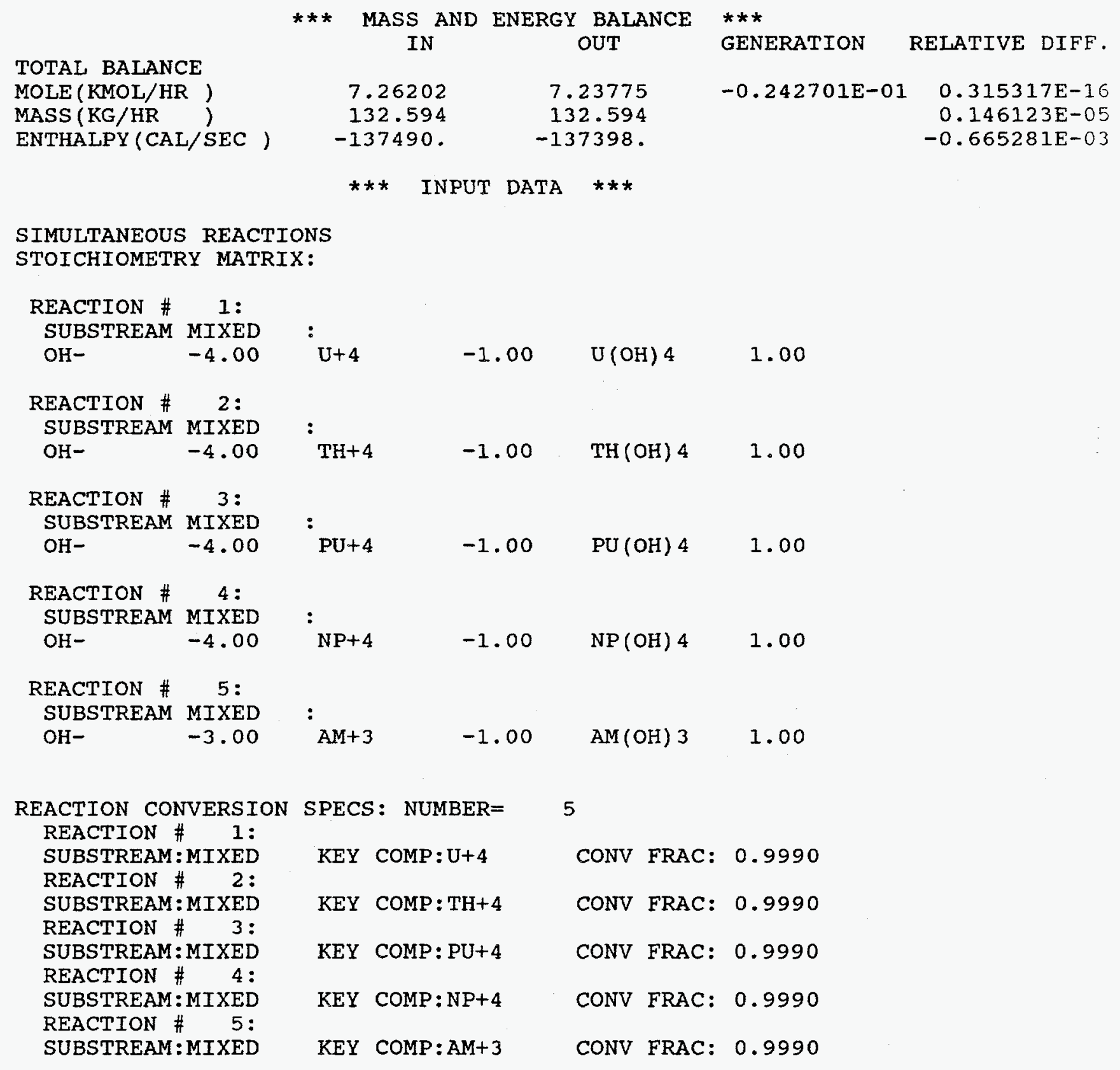


REL: $9 \cdot 1-3$

INST: LANL-PC

TANK SY-102 CLEANOUT FLOWSHEET

U-O-S BLOCK SECTION

BLOCK: TK-P-1 MODEL: RSTOIC (CONTINUED)

TWO PHASE TP FLASH

SPECIFIED TEMPERATURE $K$

SPECIFIED PRESSURE ATM

MAXIMUM NO. ITERATIONS

CONVERGENCE TOLERANCE

\author{
298.150 \\ 1.00000 \\ 30 \\ 0.000100000
}

298.15

1.0000

91.268

$0.00000 E+00$

\section{V-L PHASE EQUILIBRIUM :}

COMP

$\mathrm{H} 2 \mathrm{O}$

HNO3

NAt

$\mathrm{H} 3 \mathrm{O}+$

$\mathrm{OH}-$

NO3-

$\mathrm{TH}+4$

$\mathrm{U}+4$

$\mathrm{PU}+4$

$\mathrm{PU}(\mathrm{OH}) 4$

$\mathrm{U}(\mathrm{OH}) 4$

$\mathrm{NP}+4$

$\mathrm{AM}+3$

$\mathrm{TH}(\mathrm{OH}) 4$

$\mathrm{AM}(\mathrm{OH}) 3$

$\mathrm{NP}(\mathrm{OH}) 4$
F(I)

0.98950

$0.26964 \mathrm{E}-08$

$0.60106 \mathrm{E}-02$

$0.13351 \mathrm{E}-03$

$0.26573 E-02$

$0.86350 E-03$

$0.41443 E-06$

$0.41444 \mathrm{E}-06$

$0.95386 \mathrm{E}-08$

$0.95290 E-05$

$0.41403 E-03$

$0.17431 \mathrm{E}-09$

$0.76745 \mathrm{E}-09$

$0.41401 \mathrm{E}-03$

$0.76668 \mathrm{E}-06$

$0.17413 E-06$
$\mathrm{X}(\mathrm{I})$

0.98976

$0.58049 \mathrm{E}-19$

$0.60106 \mathrm{E}-02$

$0.38891 \mathrm{E}-14$

$0.25238 \mathrm{E}-02$

$0.86350 E-03$

$0.41443 E-06$

$0.41444 \mathrm{E}-06$

$0.95386 \mathrm{E}-08$

$0.95290 E-05$

$0.41403 E-03$

$0.17431 \mathrm{E}-09$

$0.76745 \mathrm{E}-09$

$0.41401 E-03$

$0.76668 \mathrm{E}-06$

$0.17413 E-06$
$Y(I)$

1.0000

$0.15739 \mathrm{E}-18$

$0.00000 E+00$

$0.00000 \mathrm{E}+00$

$0.00000 \mathrm{E}+00$

$0.00000 \mathrm{E}+00$

$0.00000 \mathrm{E}+00$

$0.00000 \mathrm{E}+00$

$0.00000 \mathrm{E}+00$

$0.00000 E+00$

$0.00000 \mathrm{E}+00$

$0.00000 \mathrm{E}+00$

$0.00000 \mathrm{E}+00$

$0.00000 E+00$

$0.00000 E+00$

$0.00000 \mathrm{E}+00$
$K(I)$

$0.31888 \mathrm{E}-01$

$0.85574 \mathrm{E}-01$

$0.10373 E-35$

$0.10373 E-35$

$0.10373 E-35$

$0.10373 \mathrm{E}-35$

$0.10373 E-35$

$0.10373 \mathrm{E}-35$

$0.10373 \mathrm{E}-35$

$0.10373 E-35$

$0.10373 E-35$

$0.10373 \mathrm{E}-35$

$0.10373 \mathrm{E}-35$

$0.10373 E-35$

$0.10373 E-35$

$0.10373 \mathrm{E}-35$ 
BLOCK: TK-S-1 MODEL: MIXER

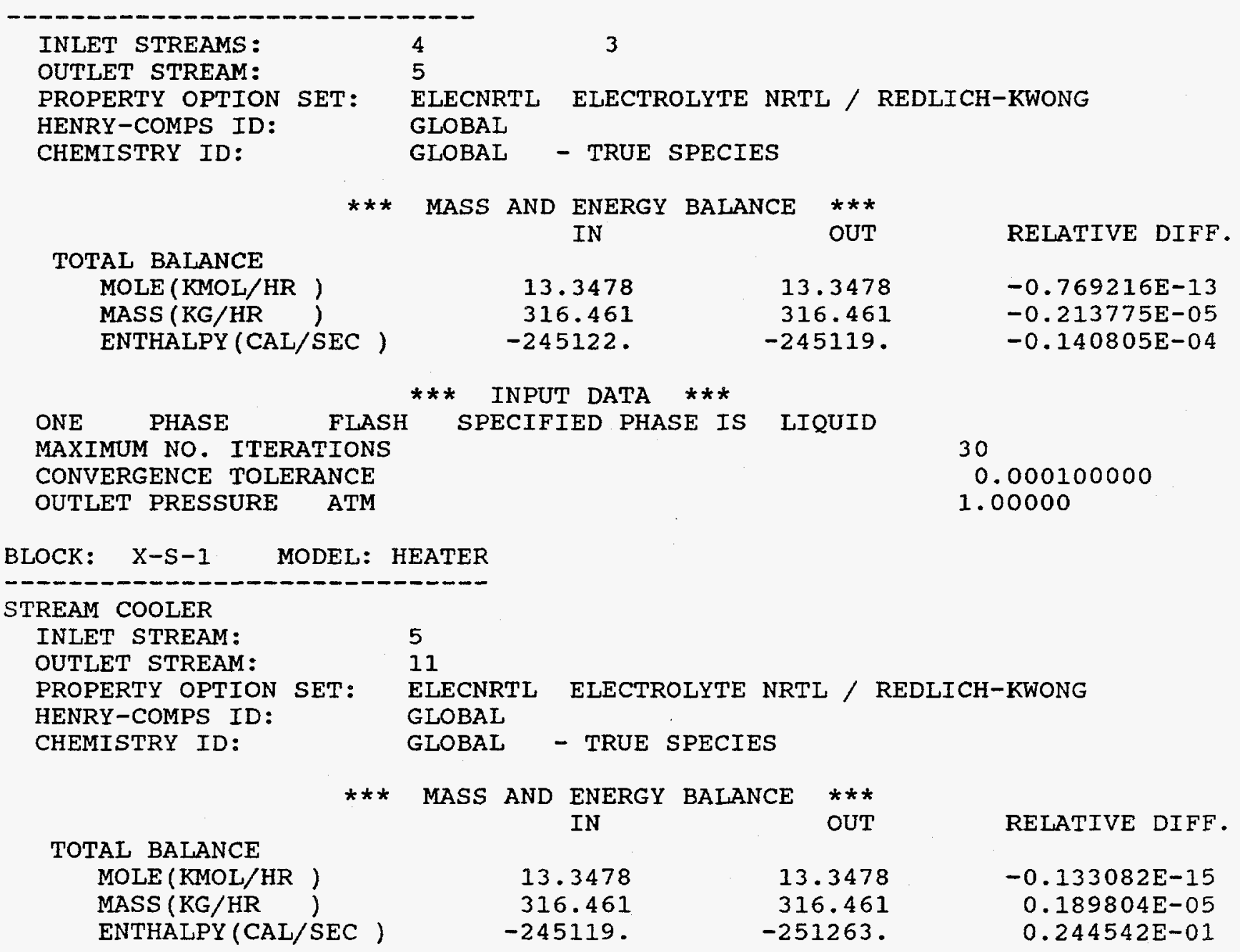


ASPEN PLUS VER: DOS

REL: 9.1-3 INST: LANL-PC

$02 / 03 / 95$

PAGE 49

TANK SY-102 CLEANOUT FLOWSHEET

U-O-S BLOCK SECTION

BLOCK: $\mathrm{X}-\mathrm{S}-1$ MODEL: HEATER (CONTINUED)

*** INPUT DATA $* * *$

TWO PHASE TF FLASH

SPECIFIED TEMPEFATURE K

SPECIFIED PRESSURE $\quad$ ATM

MAXIMUM NO. ITEFATIONS

CONVERGENCE TOLERANCE

303.150
1.00000
30
0.000100000

303.15

1.0000

$-6144.9$

$0.00000 \mathrm{E}+00$

V-L PHASE EQUILTBRIUM :

$\begin{array}{lll}\text { COMP } & F(I) & X(I) \\ \text { H2O } & 0.85029 & 0.85016 \\ \text { HNO3 } & 0.11555 E-06 & 0.14904 \mathrm{E}-06 \\ \text { F- } & 0.24623 E-02 & 0.25978 E-02 \\ \text { NAt } & 0.11992 \mathrm{E}-02 & 0.11992 \mathrm{E}-02 \\ \mathrm{H} 3 \mathrm{O}+ & 0.1155 \mathrm{E}-04 & 0.14703 \mathrm{E}-03 \\ \mathrm{HF} & 0.88957 \mathrm{E}-03 & 0.75406 \mathrm{E}-03 \\ \mathrm{OH}- & 0.81000 \mathrm{E}-10 & 0.62059 \mathrm{E}-13 \\ \mathrm{NO3}- & 0.10221 & 0.10221 \\ \mathrm{CS}+ & 0.61823 \mathrm{E}-07 & 0.61823 \mathrm{E}-07 \\ \mathrm{CA}+2 & 0.25797 \mathrm{E}-02 & 0.25797 \mathrm{E}-02 \\ \mathrm{SR}+2 & 0.11285 \mathrm{E}-04 & 0.11285 \mathrm{E}-04 \\ \mathrm{MN}+2 & 0.24683 \mathrm{E}-02 & 0.24683 \mathrm{E}-02 \\ \mathrm{FE}+3 & 0.57014 \mathrm{E}-02 & 0.57014 \mathrm{E}-02 \\ \mathrm{CR}+3 & 0.53888 \mathrm{E}-02 & 0.53888 \mathrm{E}-02 \\ \mathrm{ZR}+4 & 0.47141 \mathrm{E}-04 & 0.47141 \mathrm{E}-04 \\ \mathrm{MOO4}-2 & 0.11326 \mathrm{E}-04 & 0.11326 \mathrm{E}-04 \\ \mathrm{TH}+4 & 0.22472 \mathrm{E}-03 & 0.22472 \mathrm{E}-03 \\ \mathrm{U}+4 & 0.22473 \mathrm{E}-03 & 0.22473 \mathrm{E}-03 \\ \mathrm{AL}+3 & 0.18410 \mathrm{E}-01 & 0.18410 \mathrm{E}-01 \\ \mathrm{CO} 3-2 & 0.67417 \mathrm{E}-02 & 0.67417 \mathrm{E}-02 \\ \mathrm{SO} 4-2 & 0.11183 \mathrm{E}-02 & 0.11183 \mathrm{E}-02 \\ \mathrm{PU}+4 & 0.51722 \mathrm{E}-05 & 0.51722 \mathrm{E}-05 \\ \mathrm{NP}+4 & 0.94518 \mathrm{E}-07 & 0.94518 \mathrm{E}-07 \\ \mathrm{AM}+3 & 0.41656 \mathrm{E}-06 & 0.41656 \mathrm{E}-06 \\ \mathrm{TCO} 4- & 0.20223 \mathrm{E}-09 & 0.20223 \mathrm{E}-09\end{array}$

$Y(I)$

0.99733

$0.11269 \mathrm{E}-05$

$0.00000 E+00$

$0.00000 E+00$

$0.00000 \mathrm{E}+00$

$0.26644 \mathrm{E}-02$

$0.00000 \mathrm{E}+00$

$0.00000 E+00$

$0.00000 \mathrm{E}+00$

$0.00000 E+00$

$0.00000 \mathrm{E}+00$

$0.00000 \mathrm{E}+00$

$0.00000 E+00$

$0.00000 E+00$

$0.00000 \mathrm{E}+00$

$0.00000 E+00$

$0.00000 E+00$

$0.00000 \mathrm{E}+00$

$0.00000 E+00$

$0.00000 E+00$

$0.00000 E+00$

$0.00000 E+00$

$0.00000 E+00$

$0.00000 E+00$

$0.00000 E+00$
$\mathrm{K}(\mathrm{I})$

0.4374 OE-O 1

0.28191

$0.10356 E-35$

$0.10356 \mathrm{E}-35$

$0.10356 \mathrm{E}-35$

0.13175

$0.10356 \mathrm{E}-35$

$0.10356 \mathrm{E}-35$

$0.10356 \mathrm{E}-35$

$0.10356 E-35$

$0.10356 \mathrm{E}-35$

$0.10356 \mathrm{E}-35$

$0.10356 \mathrm{E}-35$

$0.10356 \mathrm{E}-35$

$0.10356 \mathrm{E}-35$

$0.10356 \mathrm{E}-35$

$0.10356 \mathrm{E}-35$

$0.10356 \mathrm{E}-35$

$0.10356 \mathrm{E}-35$

$0.10356 E-35$

$0.10356 E-35$

$0.10356 \mathrm{E}-35$

$0.10356 \mathrm{E}-35$

$0.10356 \mathrm{E}-35$

$0.10356 \mathrm{E}-35$ 
STREAM ID

FROM :

TO :

SUBSTREAM : MTXED

PHASE :

COMPONENTS: $\mathrm{KG} / \mathrm{HR}$

$\mathrm{H} 2 \mathrm{O}$

HNO3

$\mathrm{NAOH}$

F-

CL-

$\mathrm{H} 3 \mathrm{O}+$

HF

$\mathrm{HCL}$

NACL (S)

NANO3 (S)

$\operatorname{NAF}(S)$

$\mathrm{OH}-$

NO3 -

CS+

$\mathrm{CA}+2$

$\mathrm{SR}+2$

$\mathrm{MN}+2$

$\mathrm{FE}+3$

$\mathrm{CR}+3$

$\mathrm{ZR}+4$

MOO4-2

$\mathrm{TH}+4$

$\mathrm{U}+4$

$\mathrm{AL}+3$

CO3-2

PO4-3

SO 4-2

NO2-

HNO2

NANO2 (S)

$\mathrm{CSOH}(\mathrm{S}$ )

$\mathrm{PU}+4$

$\mathrm{PU}(\mathrm{OH}) 4$

$\mathrm{U}(\mathrm{OH}) 4$

$\mathrm{NP}+4$

$\mathrm{AM}+3$

$\mathrm{TH}(\mathrm{OH}) 4$

$\mathrm{AM}(\mathrm{OH}) 3$

$\mathrm{NP}(\mathrm{OH}) 4$

$\mathrm{TCO} 4$ -

PUO2

$\mathrm{UO} 2$

$\mathrm{AM} 2 \mathrm{O} 3$

$\mathrm{NPO} 2$

THO2
1

---

$\mathrm{C}-\mathrm{S}-1$

LIQUID

891.0000

0.0

0.0

1.4200

1.3300

36.8000

0.0

0.0

0.0

0.0

0.0

0.0

52.8000

46.5000

3. 3900-04

1.3800

1. 3198-02

1.8100

4.2500

3.7400

5.7401-02

$2.4178-02$

0.6960

0.7140

7.6900

7.6500

8.9000

2.0100

7.2500

2. $0066-11$

0.0

0.0

1. 6500-02

0.0

0.0

$2.9900-04$

1. 3400-03

0.0

0.0

0.0

$4.4000-03$

0.0

0.0

0.0

0.0

0.0
2

C-S -1

IX $-\mathrm{C}-1$

LIQUID

747.9945

0.0

0.0

0.5699

1. 3300

36.4320

0.0

0.0

0.0

0.0

0.0

0.0

29.8003

46.5000

$3.0510-04$

0.0

0.0

0.0

0.0

0.0

0.0

0.0

0.0

0.0

1. 0596

2. 2498

8.9000

0.5760

7.2500

2. $6367-11$

0.0

0.0

0.0

0.0

0.0

0.0

0.0

0.0

0.0

0.0

4. 3996-03

0.0

0.0

0.0

0.0

0.0
3
$C-S-1$
$T K-S-1$

IIQUID

143.0055

0.0

0.0

0.8500

0.0

0.3680

0.0

0.0

0.0

0.0

0.0

0.0

22.9996

0.0

1. 0967-04

1.3800

1. 3198-02

1.8100

4.2500

3.7400

5.7401-02

2. 4178-02

0.6960

0.7140

6.6303

5.4001

0.0

1.4339

0.0

0.0

0.0

0.0

1. 6500-02

0.0

0.0

2.9900-04

1. 3400-03

0.0

0.0

0.0

4. 4000-07

0.0

0.0

0.0

0.0

0.0
4

---

TK-S-1

5

TK-S -1

$\mathrm{X}-\mathrm{S}-1$

IIQUID LIQUID

$17.4226 \quad 204.4646$

$17.14369 .7185-0.5$

0.0

0.0

0.0

0.0

20.7782

0.0

0.0

0.0

0.0

0.0

0.0

67.7260

0.0

0.0

0.0

0.0

0.0

0.0

0.0

0.0

0.0

0.0

0.0

0.0

0.0

0.0

0.0

0.0

0.0

0.0

0.0

0.0

0.0

0.0

0.0

0.0

0.0

0.0

0.0

0.0

0.0

0.0

0.0

0.0

0.0

0.6244

0.0

0.3680

2. $9331-03$

0.2375

0.0

0.0

0.0

0.0

1. 8388-08

84.5955

1. 0967-04

1.3800

1. 3198-02

1.8100

4.2500

3.7400

5. 7401-02

2. 4178-02

0.6960

0.7140

6.6303

5.4001

0.0

1. 4339

0.0

0.0

0.0 
$\begin{array}{llllll}1 & 2 & 3 & 4 & 5 & \text { (CONTINUED) }\end{array}$

STREAM ID
TOTAL FLOW:
KMOL/HR
KG/HR
L/MIN
STATE VARIABLES:
TEMP K
PRES ATM
VFRAC
LFRAC
SFRAC
ENTHALPY:
CAL/MOL
CAL/GM
CAL/SEC
ENTROPY:
CAL/MOL-K
CAL/GM-K
DENSITY:
MOL/CC
GM/CC
AVG MW

1

$$
\begin{aligned}
55.9325 \\
1076.0573 \\
15.0594 \\
\\
298.1500 \\
1.0000 \\
0.0 \\
1.0000 \\
0.0
\end{aligned}
$$

$$
-6
$$

$\begin{array}{rrrr}46.0083 & 9.9241 & 3.4236 & 13.3478 \\ 882.6671 & 193.3905 & 123.0700 & 316.4612 \\ 13.1134 & 1.9977 & 1.4639 & 3.9689 \\ & & & \\ 298.1500 & 298.1500 & 298.1500 & 384.5468 \\ 1.0000 & 1.0000 & 1.0000 & 1.0000 \\ 0.0 & 0.0 & 0.0 & 0.0 \\ 1.0000 & 1.0000 & 1.0000 & 1.0000 \\ 0.0 & 0.0 & 0.0 & 0.0\end{array}$

4

5

$-6.7786+04-6.7599+04-6.8550+04-5.9043+04-6.6110+04$

$\begin{array}{lllll}-3523.4577 & -3523.5651 & -3517.7290 & -1642.5221 & -2788.4247\end{array}$

$-1.0532+06-8.6393+05-1.8897+05-5.6151+04-2.4512+05$

$$
\begin{array}{rr}
-38.9767 & -38.6658 \\
-2.0259 & -2.0154 \\
& \\
6.1902-02 & 5.8475-02 \\
1.1909 & 1.1218 \\
19.2385 & 19.1849
\end{array}
$$$$
-40.5118
$$$$
-53.7605
$$$$
-1.4955
$$$$
-37.4862
$$$$
-1.5811
$$
8. 2794-02
1.6134 19.4869
3.8979-02
1.4011
35.9466
5. 6052-02 1.3289 23.7088


REL: $9 \cdot 1-3$

INST: LANL-PC

$02 / 03 / 95$

PAGE 52 TANK SY-102 CLEANOUT FLOWSHEET

\section{STREAM SECTION}

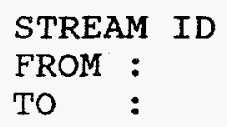

\section{SUBSTREAM : MIXED}

PHASE :

COMPONENTS: $\mathrm{KG} / \mathrm{HR}$

$\mathrm{H} 2 \mathrm{O}$

$\mathrm{HNO} 3$

$\mathrm{NAOH}$

F-

CL-

$\mathrm{NA}+$

$\mathrm{H} 3 \mathrm{O}+$

$\mathrm{HF}$

HCL

NACL (S)

NANO3 (S)

NAF (S)

$\mathrm{OH}-$

NO3-

$\mathrm{CS}+$

$\mathrm{CA}+2$

$\mathrm{SR}+2$

$\mathrm{MN}+2$

$\mathrm{FE}+3$

$\mathrm{CR}+3$

$\mathrm{ZR}+4$

MOO4-2

$\mathrm{TH}+4$

$\mathrm{U}+4$

$\mathrm{AL}+3$

CO3-2

PO4-3

SO4-2

NO2 -

HNO2

NANO2 (S)

$\mathrm{CSOH}(\mathrm{S})$

PU+ 4

$\mathrm{PU}(\mathrm{OH}) 4$

$\mathrm{U}(\mathrm{OH}) 4$

$\mathrm{NP}+4$

$\mathrm{AM}+3$

TH (OH) 4

$\mathrm{AM}(\mathrm{OH}) 3$

$\mathrm{NP}(\mathrm{OH}) 4$

$\mathrm{TCO} 4$ -

PUO2

UO2

AM2O3

NPO2

THO2

$\begin{array}{lllll}11 & 12 & 13 & 14 & 15 \\ X-S-1 & --- & I X-A-1 & I X-A-1 & \text { IX-S-1 } \\ I X-A-1 & I X-A-1 & \text { TK-P-1 } & \text { IX-S-1 } & ---\end{array}$

$$
\begin{gathered}
\text { LIQUID } \\
204.4321 \\
1.2536-04 \\
0.0 \\
0.6587 \\
0.0 \\
0.3680 \\
3.7333-02 \\
0.2013 \\
0.0 \\
0.0 \\
0.0 \\
0.0
\end{gathered}
$$

1. 4088-11

84.5954

$1.0967-04$

1.3800

1. 3198-02

1.8100

4.2500

3.7400

5.7401-02

2. 4178-02

0.6960

0.7140

6.6303

5.4001

0.0

1. 4339

0.0

0.0

0.0

0.0

1. 6500-02

0.0

0.0

$2.9900-04$

1. 3400-03

0.0

0.0

0.0

4. 4000-07

0.0

0.0

0.0

0.0

0.0

\section{LIQUID}

124.4937

1. 1651-05

0.0

0.0

0.0

0.0

0.1188

0.0

0.0

0.0

0.0

0.0

6. 2626-13

0.3874

0.0

0.0

0.0

0.0

0.0

0.0

0.0

0.0

0.0

0.0

0.0

0.0

0.0

0.0

0.0

0.0

0.0

0.0

0.0

0.0

0.0

0.0

0.0

0.0

0.0

0.0

0.0

0.0

0.0

0.0

0.0

0.0
LIQUID

124.7003

$1.2298-06$

0.0

0.0

0.0

0.0

1. 8382-02

0.0

0.0

0.0

0.0

0.0

8. 5253-12

0.3875

0.0

0.0

0.0

0.0

0.0

0.0

0.0

0.0

0.6960

0.7140

0.0

0.0

0.0

0.0

0.0

0.0

0.0

0.0

1. 6500-02

0.0

0.0

2. $9900-04$

1. 3387-03

0.0

0.0

0.0

0.0

0.0

0.0

0.0

0.0

0.0

\section{LIQUID}

204.2980

1.9975-04

0.0

0.5822

0.0

0.3680

6. $1140-02$

0.2819

0.0

0.0

0.0

0.0

$7.6053-12$

84.5952

$1.2370-04$

1.3800

$1.3198-02$

1.8100

4.2500

3.7400

5.7401-02

2. 4178-02

0.0

0.0

6.6303

5.4001

0.0

1. 4339

0.0

0.0

0.0

0.0

0.0

0.0

0.0

0.0

1. $3400-06$

0.0

0.0

0.0

4. 4000-07

0.0

0.0

0.0

0.0

0.0

\section{LIQUID}

2. 0430-02

0.0

0.0

0.0

0.0

0.0

5. 5792-10

0.0

0.0

0.0

0.0

0.0

4. $9881-10$

0.0

0.0

0.0

1.3197-02

0.0

0.0

0.0

0.0

0.0

0.0

0.0

0.0

0.0

0.0

0.0

0.0

0.0 
ASPEN PLUS VER: DOS

REL: 9.1-3 INST: LANL-PC

$02 / 03 / 95$

PAGE 53

TANK SY-102 CLEANOUT FLOWSHEET

STREAM SECTION

$\begin{array}{llllll}11 & 12 & 13 & 14 & 15 & \text { (CONTINUED) }\end{array}$

\begin{tabular}{|c|c|c|c|c|c|}
\hline STREAM ID & 11 & 12 & 13 & 14 & 15 \\
\hline \multicolumn{6}{|l|}{ TOTAL FLOW: } \\
\hline KMOL/HR & 13.3478 & 6.9229 & 6.9352 & 13.3355 & $1.2846-03$ \\
\hline KG / HR & 316.4606 & 125.0000 & 126.5344 & 314.9262 & $3.3627-02$ \\
\hline L/MIN & 3.7832 & 2.0848 & 2.0919 & 3.7651 & $3.4180-04$ \\
\hline \multicolumn{6}{|c|}{ STATE VARIABLES: } \\
\hline TEMP & 303.1500 & 298.1500 & 301.7342 & 301.7342 & 301.7342 \\
\hline PRES ATM & 1.0000 & 1.0000 & 1.0000 & 1.0000 & 1.0000 \\
\hline VFRAC & 0.0 & 0.0 & 0.0 & 0.0 & 0.0 \\
\hline LFRAC & 1.0000 & 1.0000 & 1.0000 & 1.0000 & 1.0000 \\
\hline SFRAC & 0.0 & 0.0 & 0.0 & 0.0 & 0.0 \\
\hline \multicolumn{6}{|l|}{ ENTHALPY: } \\
\hline $\mathrm{CAL} / \mathrm{MOL}$ & $-6.7768+04$ & $-6.8294+04$ & $-6.8310+04$ & $-6.7750+04$ & $-7.5410+04$ \\
\hline $\mathrm{CAL} / \mathrm{GM}$ & -2858.3281 & -3782.3511 & -3744.0069 & -2868.8620 & -2880.8724 \\
\hline $\mathrm{CAL} / \mathrm{SEC}$ & $-2.5126+05$ & $-1.3133+05$ & $-1.3160+05$ & $-2.5097+05$ & -26.9096 \\
\hline \multicolumn{6}{|l|}{ ENTROPY: } \\
\hline CAL/MOL-K & -41.6491 & -38.9901 & -38.7706 & -41.7456 & -33.5168 \\
\hline $\mathrm{CAL} / \mathrm{GM}-\mathrm{K}$ & -1.7566 & -2.1594 & -2.1249 & -1.7677 & -1.2804 \\
\hline \multicolumn{6}{|l|}{ DENSITY: } \\
\hline $\mathrm{MOL} / \mathrm{CC}$ & $5.8803-02$ & $5.5342-02$ & $5.5254-02$ & $5.9031-02$ & $6.2641-02$ \\
\hline $\mathrm{GM} / \mathrm{CC}$ & 1.3941 & 0.9992 & 1.0081 & 1.3940 & 1.6396 \\
\hline AVG MW & 23.7088 & 18.0558 & 18.2452 & 23.6155 & 26.1760 \\
\hline
\end{tabular}


STREAM ID

FROM :

TO :

SUBSTREAM : MIXED

PHASE :

COMPONENTS: $\mathrm{KG} / \mathrm{HR}$

$\mathrm{H} 2 \mathrm{O}$

HNO3

$\mathrm{NAOH}$

F-

CL-

$\mathrm{NA}+$

$\mathrm{H} 3 \mathrm{O}+$

$\mathrm{HF}$

HCL

NACL (S)

NANO3 (S)

$\mathrm{NAF}(\mathrm{S})$

$\mathrm{OH}-$

NO3 -

CS+

$\mathrm{CA}+2$

$\mathrm{SR}+2$

$\mathrm{MN}+2$

$\mathrm{FE}+3$

$\mathrm{CR}+3$

$\mathrm{ZR}+4$

MOO4-2

$\mathrm{TH}+4$

$\mathrm{U}+4$

$\mathrm{AL}+3$

CO3-2

PO4-3

SO 4-2

NO2-

HNO2

NANO2 (S)

$\mathrm{CSOH}$ (S)

PU+4

$\mathrm{PU}(\mathrm{OH}) 4$

$\mathrm{U}(\mathrm{OH}) 4$

$\mathrm{NP}+4$

$\mathrm{AM}+3$

$\mathrm{TH}(\mathrm{OH}) 4$

$\mathrm{AM}(\mathrm{OH}) 3$

$\mathrm{NP}(\mathrm{OH}) 4$

TCO4-

PUO2

$\mathrm{UO} 2$

$\mathrm{AM} 2 \mathrm{O} 3$

$\mathrm{NPO} 2$

THO2
16

$\mathrm{IX}-\mathrm{S}-1$

TK-G-1

17

TK-P-1

18
TK-P-1
$F-A-1$

19

F-A-1

TK-G-I

20

$P-A-1$

LIQUID

LIQUID

LIQUID

204.2776
$1.9975-04$
0.0
0.5822
0.0
0.3680

6. $1138-02$

0.2819

0.0

0.0

0.0

0.0

$7.6043-12$

84.5952

1. 7078-04

1.3800

1. 3198-06

1.8100

4.2500

3.7400

5. 7401-02

2. 4178-02

0.0

0.0

6.6303

5.4001

0.0

1. 4339

0.0

0.0

0.0

0.0

0.0

0.0

0.0

0.0

1. 3400-06

0.0

0.0

0.0

4. 4000-07

0.0

0.0

0.0

0.0

0.0
4.3200

0.0

0.0

0.0

0.0

1.0001
0.0

0.0

0.0

0.0

0.0

0.0

0.7399

0.0

0.0

0.0

0.0

0.0

0.0

0.0

0.0

0.0

0.0

0.0

0.0

0.0

0.0

0.0

0.0

0.0

0.0

0.0

0.0

0.0

0.0

0.0

0.0

0.0

0.0

0.0

0.0

0.0

0.0

0.0

0.0

0.0

129.0551
0.0
0.0
0.0
0.0
1.0001

5. 3548-13

0.0

0.0

0.0

0.0

0.0

0.3106

0.3875

0.0

0.0

0.0

0.0

0.0

0.0

0.0

0.0

6. 9600-04

7. 1400-04

0.0

0.0

0.0

0.0

0.0

0.0

0.0

0.0

1.6500-05

2. 1173-02

0.9171

2. $9900-07$

1.3387-06

0.8989

1. $6203-03$

3. 8440-04

0.0

0.0

0.0

0.0

0.0

0.0

\subsection{1}

0.0

0.0

0.0

0.0

1.0001

5. $4421-13$

0.0

0.0

0.0

0.0

0.0

0.3106

0.3875

0.0

0.0

0.0

0.0

0.0

0.0

0.0

0.0

6. 9600-04

7. 1400-04

0.0

0.0

0.0

0.0

0.0

0.0

0.0

0.0

1. $6500-05$

0.0

0.0

2. $9900-07$

1. $3387-06$

0.0

0.0

0.0

0.0

0.0

0.0

0.0

0.0

0.0
0.1290

0.0

0.0

0.0

0.0

0.0

1. 4639-13

0.0

0.0

0.0

0.0

0.0

2. $9863-10$

0.0

0.0

0.0

0.0

0.0

0.0

0.0

0.0

0.0

0.0

0.0

0.0

0.0

0.0

0.0

0.0

0.0

0.0

0.0

0.0

2. 1173-02

0.9171

0.0

0.0

0.8989

$1.6203-03$

3. 8440-04

0.0 
STREAM SECTION

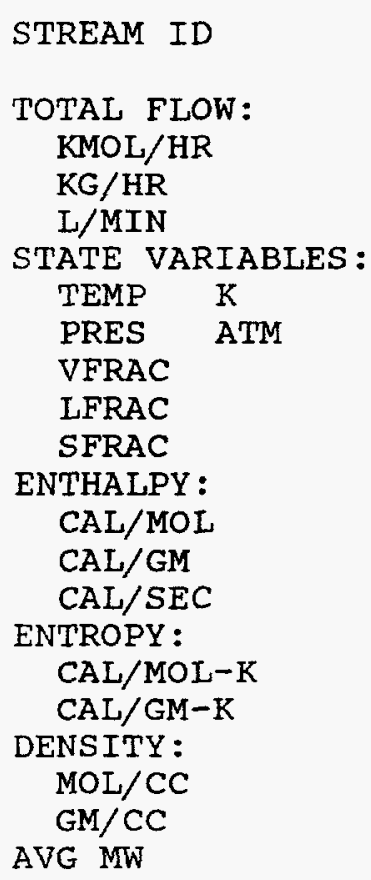

$$
16
$$

17

$$
\begin{aligned}
13.3342 \\
314.8925 \\
3.7647 \\
301.7342 \\
1.0000 \\
0.0 \\
1.0000 \\
0.0
\end{aligned}
$$

$$
\begin{aligned}
0.3268 \\
6.0600 \\
7.7088-02 \\
298.1500 \\
1.0000 \\
0.0 \\
1.0000 \\
0.0
\end{aligned}
$$

18

19

20

$$
\begin{array}{rr}
-6.7749+04 & -6.4924+04 \\
-2868.8608 & -3501.2236 \\
-2.5094+05 & -5893.7265 \\
-41.7468 & -34.0875 \\
-1.7677 & -1.8382 \\
& \\
5.9031-02 & 7.0655-02 \\
1.3940 & 1.3101 \\
23.6153 & 18.5433
\end{array}
$$
7. 0655-02
1.3101

$$
\begin{aligned}
7.2377 \\
132.5942 \\
2.1704 \\
298.1500 \\
1.0000 \\
0.0 \\
1.0000 \\
0.0
\end{aligned}
$$$$
-6.8341+04
$$$$
-3730.4329
$$$$
-1.3740+05
$$

$-6.8195+04-$

7.2245

130.6258

2.1549

$1.3233-02$

1.9683

298.1500

1.0000

0.0

1.0000

0.0
$-39.1084$
$-2.1347$
5. 5579-02
1. 0182
18.3198

$5.5876-02$
1.0102
18.0809

1. 4246-02

2.1190

148.7489 


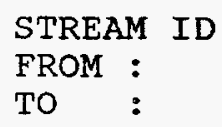

THO2
21

SPLIT

-- -

VAPOR

\subsection{2}

0.0

0.0

0.0

0.0

0.0

0.0

0.0

0.0

0.0

0.0

0.0

0.0

0.0

0.0

0.0

0.0

0.0

0.0

0.0

0.0

0.0

0.0

0.0

0.0

0.0

0.0

0.0

0.0

0.0

0.0

0.0

0.0

0.0

0.0

0.0

0.0

0.0

0.0

0.0

0.0

0.0

0.0

0.0

0.0

0.0

\section{2 \\ SPLIT}

LIQUID

$4.1992-04$

0.0

0.0

0.0

0.0

0.0

0.0

0.0

0.0

0.0

0.0

0.0

$3.4713-08$

0.0

0.0

0.0

0.0

0.0

0.0

0.0

0.0

0.0

0.0

0.0

0.0

0.0

0.0

0.0

0.0

0.0

0.0

0.0

0.0

0.0

0.0

0.0

0.0

0.0

0.0

0.0

0.0

1. 9040-02

0.8091

2. 3910-04

3. 3909-04

0.7911
24
$I X-C-1$

25
IX-C-I
IX $-T-1$

26

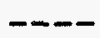

LIQUID

LIQUID

LIQUID

7. 4799-03

0.0

0.0

0.0

0.0

0.0

1. $9425-11$

0.0

0.0

0.0

0.0

0.0

1. $7363-11$

0.0

3. 0507-04

0.0

0.0

0.0

0.0

0.0

0.0

0.0

0.0

0.0

0.0

0.0

0.0

0.0

0.0

0.0

0.0

0.0

0.0

0.0

0.0

0.0

0.0

0.0

0.0

0.0

0.0

0.0

0.0

0.0

0.0

0.0
747.9870

0.0

0.0

0.5699

1.3300

36.4320

0.0

0.0

0.0

0.0

0.0

0.0

29.8003

46.5000

$3.0516-08$

0.0

0.0

0.0

0.0

0.0

0.0

0.0

0.0

0.0

1.0596

2. 2498

8.9000

0.5760

7.2500

2. 6366-11

0.0

0.0

0.0

0.0

0.0

0.0

0.0

0.0

0.0

0.0

4. 3996-03

0.0

0.0

0.0

0.0

0.0
7. 4799-03

0.0

0.0

0.0

0.0

0.0

8. 9899-12

0.0

0.0

0.0

0.0

0.0

$8.0367-12$

0.0

0.0

0.0

0.0

0.0

0.0

0.0

0.0

0.0

0.0

0.0

0.0

0.0 
ASPEN PLUS VER: DOS

REL: 9.1-3 INST: LANL-PC

02/03/95 PAGE 57

TANK SY-102 CLEANOUT FLOWSHEET

STREAM SECTION

$\begin{array}{llllll}21 & 22 & 24 & 25 & 26 & \text { (CONTINUED) }\end{array}$

STREAM ID

21

22

24

25

26

TOTAL FLOW:

KMOL/HR

KG/HR

L/MIN

STATE VARIABLES :

TEMP $K$

PRES ATM

VFRAC

LFRAC

SFRAC

ENTHALPY :

CAL/MOL

CAL/GM

CAL/SEC

ENTROPY :

CAL/MOL $-\mathrm{K}$

CAL/GM-K

DENSITY :

MOL/CC

$\mathrm{GM} / \mathrm{CC}$

AVG MW

$$
\begin{array}{r}
1.9275-02 \\
0.3472 \\
24.3223 \\
\\
923.1500 \\
1.0000 \\
1.0000 \\
0.0 \\
0.0
\end{array}
$$

6.0895-03

1.6204

4.1750-04

7.7850-03

3.7545-02

1. 2502-04

46.0079

882.6593

4. 4218-04

13.1132

1. 1879-02
923.1500
1.0000
0.0
1.0000
0.0

298.1500

1.0000

0.0

1.0000

0.0
298.1500
1.0000
0.0
1.0000
0.0

1. 8427-04

$$
\begin{array}{r}
-5.2342+04 \\
-2905.3990 \\
-280.2515 \\
-0.8895 \\
-4.9379-02 \\
\\
1.3208-05 \\
2.3795-04 \\
18.0152
\end{array}
$$$$
-6.8275+04
$$$$
\begin{array}{r}
-1.0650+05 \\
-400.2127
\end{array}
$$$$
\begin{aligned}
-36275+04 & -6.7599+04 \\
-3661.4325 & -3523.5639
\end{aligned}
$$$$
-180.1403
$$$$
-7.9178
$$

$-8.6392+05$

$-6.4602+04$

$$
\begin{array}{r}
164.5436 \\
0.6183
\end{array}
$$$$
\begin{array}{r}
-38.5815 \\
-2.0690
\end{array}
$$$$
-38.6658
$$$$
-2.0154
$$

298.1500

1.0000

0.0

1.0000

0.0

2.7032-03

0.7193

5. 5657-02

1.0378

266.0994

18.6469
5. 8475-02
1.1218
19.1849

$3.9994-02$
1.0744
26.8643

$-7.9350$

$-24.1616$

$-0.8993$ 


\begin{tabular}{|c|c|c|}
\hline $\begin{array}{l}\text { STREAM ID } \\
\text { FROM : } \\
\text { TO : }\end{array}$ & $\begin{array}{l}27 \\
I X-T-1 \\
T K-G-1\end{array}$ & $\begin{array}{l}29 \\
\text { TK-G-1 } \\
----\end{array}$ \\
\hline SUBSTREAM: MIXED & & \\
\hline PHASE: & LIQUID & LIQUID \\
\hline COMPONENTS: KG/HR & & \\
\hline $\mathrm{H} 2 \mathrm{O}$ & 747.9795 & 1081.5530 \\
\hline HNO3 & 0.0 & 0.0 \\
\hline $\mathrm{NAOH}$ & 0.0 & 0.0 \\
\hline$F-$ & 0.5699 & 1.4200 \\
\hline $\mathrm{CL}-$ & 1.3300 & 1.3300 \\
\hline NAt & 36.4320 & 37.8001 \\
\hline $\mathrm{H} 3 \mathrm{O}+$ & 0.0 & 0.0 \\
\hline $\mathrm{HF}$ & 0.0 & $3.3631-12$ \\
\hline $\mathrm{HCL}$ & 0.0 & 0.0 \\
\hline $\operatorname{NACL}(S)$ & 0.0 & 0.0 \\
\hline NANO3 $(\mathrm{S})$ & 0.0 & 0.0 \\
\hline $\operatorname{NAF}(S)$ & 0.0 & 0.0 \\
\hline $\mathrm{OH}^{-}$ & 29.8003 & 29.8165 \\
\hline NO3- & 46.5000 & 131.4830 \\
\hline $\operatorname{cst}$ & $3.0562-08$ & $2.0483-04$ \\
\hline $\mathrm{CA}+2$ & 0.0 & 1.3800 \\
\hline $\mathrm{SR}+2$ & 0.0 & $1.3198-06$ \\
\hline $\mathrm{MN}+2$ & 0.0 & 1.8100 \\
\hline $\mathrm{FE}+3$ & 0.0 & 4.2500 \\
\hline$C R+3$ & 0.0 & 3.7400 \\
\hline $\mathrm{ZR}+4$ & 0.0 & $5.7401-02$ \\
\hline $\mathrm{MOO}_{4-2}$ & 0.0 & $2.4178-02$ \\
\hline $\mathrm{TH}+4$ & 0.0 & $6.9600-04$ \\
\hline$U+4$ & 0.0 & $7.1400-04$ \\
\hline$A L+3$ & 1.0596 & 7.6900 \\
\hline $\mathrm{CO} 3-2$ & 2.2498 & 7.6500 \\
\hline $\mathrm{PO} 4-3$ & 8.9000 & 8.9000 \\
\hline $\mathrm{SO} 4-2$ & 0.5760 & 2.0100 \\
\hline NO2- & 7.2500 & 7.2500 \\
\hline HNO2 & $2.6366-11$ & $6.5175-11$ \\
\hline NANO2 (S) & 0.0 & 0.0 \\
\hline $\mathrm{CSOH}(\mathrm{S})$ & 0.0 & 0.0 \\
\hline $\mathrm{PU}+4$ & 0.0 & $1.6500-05$ \\
\hline $\mathrm{PU}(\mathrm{OH}) 4$ & 0.0 & 0.0 \\
\hline $\mathrm{U}(\mathrm{OH}) 4$ & 0.0 & 0.0 \\
\hline $\mathrm{NP}+4$ & 0.0 & $2.9900-07$ \\
\hline$A M+3$ & 0.0 & $2.6787-06$ \\
\hline $\mathrm{TH}(\mathrm{OH}) 4$ & 0.0 & 0.0 \\
\hline $\mathrm{AM}(\mathrm{OH}) 3$ & 0.0 & 0.0 \\
\hline $\mathrm{NP}(\mathrm{OH}) 4$ & 0.0 & 0.0 \\
\hline $\mathrm{TCO}_{4-}$ & $4.3996-07$ & $8.7996-07$ \\
\hline PUO2 & 0.0 & 0.0 \\
\hline UO2 & 0.0 & 0.0 \\
\hline AM2O3 & 0.0 & 0.0 \\
\hline $\mathrm{NPO} 2$ & 0.0 & 0.0 \\
\hline THO2 & 0.0 & 0.0 \\
\hline
\end{tabular}




\author{
2729 (CONTINUED) \\ STREAM ID \\ TOTAL FLOW: \\ KMOL/HR \\ KG/HR \\ L/MIN \\ STATE VARIABLES: \\ TEMP $K$ \\ PRES ATM \\ VFRAC \\ LFRAC \\ SFRAC \\ ENTHALPY : \\ CAL/MOL \\ CAL/GM \\ CAL/SEC \\ ENTROPY : \\ CAL/MOL-K \\ CAL/GM-K \\ DENSITY: \\ MOL/CC \\ $\mathrm{GM} / \mathrm{CC}$ \\ AVG MW

(1)

(n)

27

29 